\title{
Cross-view Embeddings for Information Retrieval
}

\author{
Parth Alokkumar Gupta \\ Departamento de Sistemas Informáticos y Computación \\ Advisor: Dr. Paolo Rosso \\ Universitat Politècnica de València \\ Co-advisor: Dr. Rafael E. Banchs \\ Institute for Infocomm Research, Singapore
}

Thesis developed to obtain the Degree of Doctor por la Universitat Politècnica de València

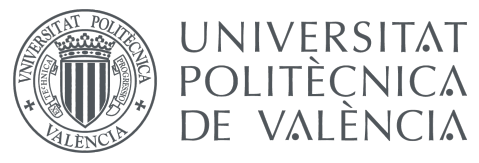

December, 2016 



\section{Abstract}

In this dissertation, we deal with the cross-view tasks related to information retrieval using embedding methods. We study existing methodologies and propose new methods to overcome their limitations. We formally introduce the concept of mixed-script IR, which deals with the challenges faced by an IR system when a language is written in different scripts because of various technological and sociological factors. Mixedscript terms are represented by a small and finite feature space comprised of character n-grams. We propose the cross-view autoencoder (CAE) to model such terms in an abstract space and CAE provides the state-of-the-art performance.

We study a wide variety of models for cross-language information retrieval (CLIR) and propose a model based on compositional neural networks (XCNN) which overcomes the limitations of the existing methods and achieves the best results for many CLIR tasks such as ad-hoc retrieval, parallel sentence retrieval and cross-language plagiarism detection. We empirically test the proposed models for these tasks on publicly available datasets and present the results with analyses.

In this dissertation, we also explore an effective method to incorporate contextual similarity for lexical selection in machine translation. Concretely, we investigate a feature based on context available in source sentence calculated using deep autoencoders. The proposed feature exhibits statistically significant improvements over the strong baselines for English-to-Spanish and English-to-Hindi translation tasks. 
Finally, we explore the the methods to evaluate the quality of autoencoder generated representations of text data and analyse its architectural properties. For this, we propose two metrics based on reconstruction capabilities of the autoencoders: structure preservation index (SPI) and similarity accumulation index (SAI). We also introduce a concept of critical bottleneck dimensionality (CBD) below which the structural information is lost and present analyses linking CBD and language perplexity. 


\section{Resumen}

En esta disertación estudiamos problemas de vistas-múltiples relacionados con la recuperación de información utilizando técnicas de representación en espacios de baja dimensionalidad. Estudiamos las técnicas existentes y proponemos nuevas técnicas para solventar algunas de las limitaciones existentes. Presentamos formalmente el concepto de recuperación de información con escritura mixta, el cual trata las dificultades de los sistemas de recuperación de información cuando los textos contienen escrituras en distintos alfabetos debido a razones tecnológicas y socioculturales. Las palabras en escritura mixta son representadas en un espacio de características finito y reducido, compuesto por n-gramas de caracteres. Proponemos los auto-codificadores de vistas-múltiples (CAE, por sus siglas en inglés) para modelar dichas palabras en un espacio abstracto, y esta técnica produce resultados de vanguardia.

En este sentido, estudiamos varios modelos para la recuperación de información entre lenguas diferentes (CLIR, por sus siglas en inglés) y proponemos un modelo basado en redes neuronales composicionales (XCNN, por sus siglas en inglés), el cual supera las limitaciones de los métodos existentes. El método de XCNN propuesto produce mejores resultados en diferentes tareas de CLIR tales como la recuperación de información ad-hoc, la identificación de oraciones equivalentes en lenguas distintas y la detección de plagio entre lenguas diferentes. Para tal efecto, realizamos pruebas experimentales para dichas tareas sobre conjuntos de datos disponibles públicamente, 
presentando los resultados y análisis correspondientes.

En esta disertación, también exploramos un método eficiente para utilizar similitud semántica de contextos en el proceso de selección léxica en traducción automática. Específicamente, proponemos características extraídas de los contextos disponibles en las oraciones fuentes mediante el uso de auto-codificadores. El uso de las características propuestas demuestra mejoras estadísticamente significativas sobre sistemas de traducción robustos para las tareas de traducción entre inglés y español, e inglés e hindú.

Finalmente, exploramos métodos para evaluar la calidad de las representaciones de datos de texto generadas por los auto-codificadores, a la vez que analizamos las propiedades de sus arquitecturas. Como resultado, proponemos dos nuevas métricas para cuantificar la calidad de las reconstrucciones generadas por los autocodificadores: el índice de preservación de estructura (SPI, por sus siglas en inglés) y el índice de acumulación de similitud (SAI, por sus siglas en inglés). También presentamos el concepto de dimensión crítica de cuello de botella (CBD, por sus siglas en inglés), por debajo de la cual la información estructural se deteriora. Mostramos que, interesantemente, la CBD está relacionada con la perplejidad de la lengua. 


\section{Resum}

En aquesta dissertació estudiem els problemes de vistes-múltiples relacionats amb la recuperació d'informació utilitzant tècniques de representació en espais de baixa dimensionalitat. Estudiem les tècniques existents i en proposem unes de noves per solucionar algunes de les limitacions existents. Presentem formalment el concepte de recuperació d'informació amb escriptura mixta, el qual tracta les dificultats dels sistemes de recuperació d'informació quan els textos contenen escriptures en diferents alfabets per motius tecnològics i socioculturals. Les paraules en escriptura mixta són representades en un espai de característiques finit i reduït, composat per n-grames de caràcters. Proposem els auto-codificadors de vistes-múltiples (CAE, per les seves sigles en anglès) per modelar aquestes paraules en un espai abstracte, i aquesta tècnica produeix resultats d'avantguarda.

En aquest sentit, estudiem diversos models per a la recuperació d'informació entre llengües diferents (CLIR, per les sevas sigles en anglès) i proposem un model basat en xarxes neuronals composicionals (XCNN, per les sevas sigles en anglès), el qual supera les limitacions dels mètodes existents. El mètode de XCNN proposat produeix millors resultats en diferents tasques de CLIR com ara la recuperació d'informació ad-hoc, la identificació d'oracions equivalents en llengües diferents, i la detecció de

plagi entre llengües diferents. Per a tal efecte, realitzem proves experimentals per aquestes tasques sobre conjunts de dades disponibles públicament, presentant els 
resultats i anàlisis corresponents.

En aquesta dissertació, també explorem un mètode eficient per utilitzar similitud semàntica de contextos en el procés de selecció lèxica en traducció automàtica. Específicament, proposem característiques extretes dels contextos disponibles a les oracions fonts mitjançant l'ús d'auto-codificadors. L'ús de les característiques proposades demostra millores estadísticament significatives sobre sistemes de traducció robustos per a les tasques de traducció entre anglès i espanyol, i anglès i hindú.

Finalment, explorem mètodes per avaluar la qualitat de les representacions de dades de text generades pels auto-codificadors, alhora que analitzem les propietats de les seves arquitectures. Com a resultat, proposem dues noves mètriques per quantificar la qualitat de les reconstruccions generades pels auto-codificadors: l'índex de preservació d'estructura (SCI, per les seves sigles en anglès) i l'índex d'acumulació de similitud (SAI, per les seves sigles en anglès). També presentem el concepte de dimensió crítica de coll d'ampolla (CBD, per les seves sigles en anglès), per sota de la qual la informació estructural es deteriora. Mostrem que, de manera interessant, la CBD està relacionada amb la perplexitat de la llengua. 


\section{Acknowledgements}

First and foremost, I want to express my gratitude for people without whom this research might not be possible.

My sincere thanks to Paolo Rosso who graciously helped me with every aspect of this journey. He has devoted a huge amount of time in training me in the field of research. I am also highly thankful to him because of having confidence in me and providing me all the opportunities to succeed in this project. The enthusiasm and trust he has shown to support me is unparalleled. He has been a fantastic advisor.

I thank Rafael E. Banchs, my co-advisor, for the his valuable effort and energy put in advising me with research. He also played an instrumental part in hosting me at Institute for Infocomm Research (8 months - Singapore) and introducing me to the aspects of industrial research. I am also thankful to him for introducing me to the world of neural networks and helping me to take some critical decisions in terms of focus of this dissertation.

Thanks to Doug Oard, Lluis Marquez and Pablo Castell, who have given their feedback as external reviewers. Their constructive and insightful comments have greatly improved the quality of this dissertation. I also thank Eneko Agirre, Julio Gonzalo and Jaap Kamps to be on the thesis defense committee.

I feel lucky to work with colleagues at PRHLT research center and DSIC like Alberto Barrón-Cedeño, Enrique Flores, Marc Franco Salvador, Francisco Rangel, Irazu 
Hernández, Maite Giménez, Joan Puigcerver and Mauricio Villegas. I also enjoyed the discussions with Jon Ander Gomez. Roberto Paredes, Francisco Casacuberta and Enrique Vidal have been very helpful by providing constructive feedback.

This work has also benefited a lot from collaborations with Marta Ruiz CostaJussá from UPC. She has been very helpful and motivating with the aspects related to machine translation. I want to thank Claudio Giuliano for hosting me at FBK research center (2 months - Italy) and providing constructive feedback on experimental setup. Collaborations with Monojit Choudhury and Kalika Bali has been very helpful in this research especially with the mixed-script information retrieval aspect. They generously hosted me at Microsoft Research (3 months - India). It is also very important for me to express my gratitude to Doug Oard and Iadh Ounis for providing me insightful comments on this work in the framework of Doctoral Consortium at SIGIR (Australia). I am thankful to ACM for providing this opportunity through ACM Travel Grant. I am very thankful to Nicola Cancedda to mentor me at Microsoft Bing (4 months - London) and introducing me to the plethora of engineering challenges involved in research and web search. Jose Santos, Andrea Moro, Daniel Voinea, Panagiotis Tigas and Bhaskar Mitra at Microsoft Bing have also been very supportive. I also want to thank Paul Clough, Mark Stevenson, Prasenjit Majumder, Mandar Mitra for helping in organising CL!NSS and MSIR research tracks at FIRE. I am also thankful to Shobha L and Vasudev Varma for hosting me at AU-KBC research center (3 months - Chennai) and IIIT-Hyderabad (2 months) respectively in the framework of WIQ-EI project.

My sincere gratitude to Neha for believing in me and standing by my side throughout this journey. The love and support has always been a source of energy. I also want to thank my parents - Alokkumar Gupta and Mithilesh Gupta - and sister Nirzari Gupta - for all the love and blessings. I am very happy that you are proud of me. I want to thank Rosa and Oreste for making me feel like home in a foreign country. During this journey, I had a chance to enjoy the friendship of Joan Pastor, Marcos Calvo, Joaquin Planells, Fernando Sánchez-Vega, Dasha Bogdanova, Ismael 
Diáz. I am also thankful to David Aguilar, Sören Volgmann and Dishant Chauhan for their friendship. The staff at UPV has been tremendously supportive in every possible way. The list of people to thank is long and I am sure to have missed some names.

Finally, my thanks to the Universitat Politècnica de València for supporting the PhD thesis through Formación de Personal Investigador (FPI) grant (№ de registro - 3505). This research has been carried out in the framework of following projects: (i) WIQ-EI IRSES project (Grant No. 269180) within the FP 7 Marie Curie; (ii) Text-Enterprise 2.0 research project (TIN2009-13391-C04-03); and (iii) DIANAAPPLICATIONS - Finding Hidden Knowledge in Texts: Applications (TIN201238603-C02-01). I gratefully acknowledge the support of NVIDIA Corporation with the donation of the GeForce Titan GPU used for this research. 



\section{Contents}

\begin{tabular}{ll}
\hline Abstract & i
\end{tabular}

Resumen iii

Resum v v

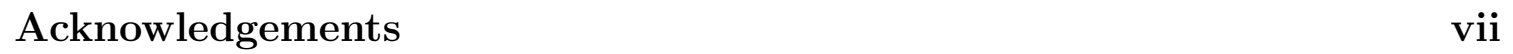

Contents $\quad x i$

List of Figures $\quad$ xvii

List of Tables $\quad$ xix

1 Introduction 1

1.1 Contributions . . . . . . . . . . . . . . . . . . . . . . . 3

1.1 .1 Mixed-script information retrieval . . . . . . . . . . . . . . 3

1.1 .2 Cross-view models . . . . . . . . . . . . . . . . 5

1.1.3 Critical bottleneck dimensionality . . . . . . . . . . . . . . 6

1.2 Research Questions (RQ) . . . . . . . . . . . . . . . . 6

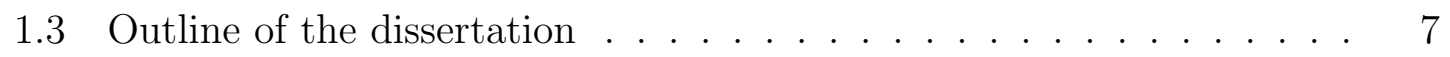


\begin{tabular}{|lll}
2 & Theoretical background & 9
\end{tabular}

2.1 Information retrieval (IR) . . . . . . . . . . . . . . . . 9

$2.1 .1 \quad$ Vector space model . . . . . . . . . . . . . . . . . 10

$2.1 .2 \quad$ Indexing and retrieval . . . . . . . . . . . . . . . . 11

2.1 .3 Evaluation . . . . . . . . . . . . . . . . . 12

2.1 .4 Semantic term-relatedness . . . . . . . . . . . . . . . 14

2.2 Dimensionality reduction . . . . . . . . . . . . . . . . . . . . . . 15

2.3 IR across languages $\ldots \ldots \ldots \ldots$. . . . . . . . . . . . . . . . . 18

2.4 IR across scripts . . . . . . . . . . . . . . . . . . . . . . . . . 20

2.5 Cross-view models . . . . . . . . . . . . . . . . . . . . . . . . 22

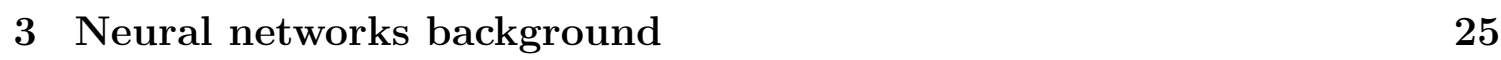

3.1 Neural networks . . . . . . . . . . . . . . . . . . . 25

3.2 Restricted Boltzmann machines (RBM) . . . . . . . . . . . . . . . . . 28

3.2.1 $\quad$ Stochastic binary RBM . . . . . . . . . . . . . . . . . . . . . 29

3.2 .2 Multinomial RBM . . . . . . . . . . . . . . . . . . . . . . . 29

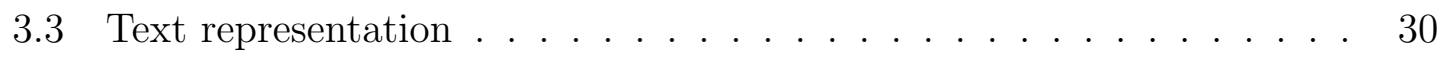

3.4 Autoencoder . . . . . . . . . . . . . . . . . . . . . . 31

3.5 Backpropagation . . . . . . . . . . . . . . . . . . 32

3.6 Optimisation . . . . . . . . . . . . . . . . 34

\begin{tabular}{lll}
\hline & Cross-view models & 37
\end{tabular}

4.1 Cross-view autoencoder (CAE) for mixed-script IR . . . . . . . . . . 38

$4.1 .1 \quad$ Formulation . . . . . . . . . . . . . . . . . . . . . . . . 39

4.1 .2 Closed feature set - finite $K$. . . . . . . . . . . . . . . 41

4.1 .3 Training . . . . . . . . . . . . . . . . . . . . . 42

(i) Layer-wise pre-training . . . . . . . . . . . . . 42

(ii) Fine tuning . . . . . . . . . . . . . . 43

$4.1 .4 \quad$ Finding equivalents . . . . . . . . . . . . . . . . . . . 44

4.2 External data composition neural networks . . . . . . . . . . . . . . . 44 
$4.2 .1 \quad$ Monolingual pre-initialisation . . . . . . . . . . . . . 46

4.2 .2 Cross-language extension . . . . . . . . . . . . . . . . . 49

5 Mixed-script information retrieval 53

$5.1 \quad$ MSIR: Definition \& challenges . . . . . . . . . . . . . . . . . . . 54

$5.1 .1 \quad$ Languages, scripts and transliteration . . . . . . . . . . . . 54

$5.1 .2 \quad$ Mixed-script IR . . . . . . . . . . . . . . . . . . . 55

$5.1 .3 \quad$ Mixed and transliterated queries \& documents . . . . . . . . . 56

$5.1 .4 \quad$ Challenges in MSIR . . . . . . . . . . . . . . . . . . . . . 57

5.2 Transliterated queries in web search . . . . . . . . . . . . . . . . 58

5.2 .1 Methodology . . . . . . . . . . . . . . . 58

Step-1: Language identification . . . . . . . . . . 59

Step-2: Query categories . . . . . . . . . . . . . 59

Step-3: Category assignment . . . . . . . . . . . . . . 59

5.2 .2 Observations . . . . . . . . . . . . . . . . . . 62

5.3 Experiments and results . . . . . . . . . . . . . . . 63

5.3 .1 Dataset . . . . . . . . . . . . . . . 63

5.3 .2 Experimental setup . . . . . . . . . . . . . . . 64

5.3 .3 Baseline systems . . . . . . . . . . . . . . 65

5.3 .4 Results and Analysis . . . . . . . . . . . . . . 66

$5.3 .5 \quad$ Scalability . . . . . . . . . . . . . . . . . . . . . 69

$\begin{array}{lll}6 & \text { Cross-language information retrieval } & 73\end{array}$

6.1 Cross-language text similarity . . . . . . . . . . . . . . . . . 74

(i) Lexical-based systems . . . . . . . . . . . . . . . 74

(ii) Thesauri-based systems. . . . . . . . . . . . . . . . 74

(iii) Comparable corpus-based systems . . . . . . . . . 74

(iv) Parallel corpus-based systems . . . . . . . . . . . 75

$(v)$ Machine translation-based systems . . . . . . . . . . 75

(vi) Translingual continuous space systems . . . . . . . 75 
$6.1 .1 \quad$ Cross-language character $n$-grams (CL-CNG) . . . . . . . . . 75

6.1 .2 Cross-language explicit semantic analysis (CL-ESA) . . . . . . 76

6.1.3 Cross-language alignment-based similarity analysis (CL-ASA) 76

6.1 .4 Cross-language knowledge graph analysis (CL-KGA) . . . . . 77

6.1 .5 Cross-language latent semantic indexing (CL-LSI) . . . . . . . 79

6.1 .6 Oriented principal component analysis (OPCA) . . . . . . . . 80

6.1.7 Similarity learning via siamese neural network (S2Net) . . . . 81

6.1 .8 Machine translation. . . . . . . . . . . . . . . . . . 82

6.1 .9 Hybrid models . . . . . . . . . . . . . . . . . 83

6.1.10 Continuous word alignment-based similarity analysis (CWASA) 84

6.2 Cross-language plagiarism detection . . . . . . . . . . . . . . . . . . . 85

6.2 .1 Problem statement . . . . . . . . . . . . . . . . . . 85

6.2 .2 Detailed analysis method . . . . . . . . . . . . . . . 86

6.2 .3 Dataset and experiments . . . . . . . . . . . . . . 87

6.2 .4 Results and analysis . . . . . . . . . . . . . . . . . 90

(i) CL-C3G . . . . . . . . . . . . . . 90

(ii) CL-ESA . . . . . . . . . . . . . . . 90

(iii) CL-ASA. . . . . . . . . . . . . . 90

(iv) CL-KGA . . . . . . . . . . . . . . . . 90

(v) S2Net, CAE, XCNN . . . . . . . . . . . . 90

6.2.4.1 Experiment A: Cross-language similarity ranking . . 91

6.2.4.2 Experiment B: Cross-language plagiarism detection . 92

6.3 Cross-language ad-hoc retrieval $\ldots \ldots$. . . . . . . . . . . . . . . . . . . . . 94

6.3 .1 Problem statement . . . . . . . . . . . . . . . . . . . 94

6.3 .2 Methods . . . . . . . . . . . . . . . . 95

$6.3 .3 \quad$ Datasets and experiments . . . . . . . . . . . . . . . . 95

6.3 .4 Results and analysis . . . . . . . . . . . . . . . . . 96

6.4 Cross-language parallel sentence retrieval . . . . . . . . . . . . . . . . 99

6.4 .1 Problem statement . . . . . . . . . . . . . . . . . . . . . . 99 
6.4 .2 Datasets and experiments . . . . . . . . . . . . . . . 99

6.4 .3 Results and analysis . . . . . . . . . . . . . . 100

6.5 Source context for machine translation . . . . . . . . . . . . . . . 100

6.5 .1 Source-context feature . . . . . . . . . . . . . . . . . 101

6.5 .2 Datasets and experiments . . . . . . . . . . . . 102

6.5 .3 Results and analysis . . . . . . . . . . . . . . . . . . 104

6.5 .4 Scalability . . . . . . . . . . . . . . . . 106

\begin{tabular}{lll}
\hline 7 & Bottleneck dimensionality for autoencoders & 109
\end{tabular}

7.1 Qualitative analysis and metrics . . . . . . . . . . . . . . 111

7.1 .1 Metrics . . . . . . . . . . . . . . . . 111

(i) Structure preservation index . . . . . . . . . . 112

(ii) Similarity accumulation index . . . . . . . . . . 112

$7.1 .2 \quad$ Comparative evaluation of models . . . . . . . . . . . . . . 113

$7.1 .3 \quad$ Analysis and discussion. . . . . . . . . . . . . . . 114

7.2 Critical bottleneck dimensionality . . . . . . . . . . . . . . . . . . 115

7.2 .1 Metric selection . . . . . . . . . . . . . . . 116

7.2 .2 Multilingual analysis . . . . . . . . . . . . . . . . . 118

7.3 Critical dimensionality and perplexity . . . . . . . . . . . . . . . 121

8 Conclusions 125

8.1 Conclusions . . . . . . . . . . . . . . . . . . . . 125

8.2 Limitations . . . . . . . . . . . . . . . . . . . . . . . . . . 128

Parallel/comparable data: . . . . . . . . . . . . 128

External relevance signals: . . . . . . . . . . . . . . . 128

Computational resources: . . . . . . . . . . . . . . 128

Mixed-script data: . . . . . . . . . . . . . . . . . . . . 129

Evaluation metrics for mixed-script terms: . . . . . . . 129

8.3 Code . . . . . . . . . . . . . . . . . . . . . . . . . . 129

8.4 Future work . . . . . . . . . . . . . . . . . . . . . . . . . . . . 129 
$8.4 .1 \quad$ Mixed-script IR . . . . . . . . . . . . . . . . . . . . . . . 129

8.4 .2 Composition neural networks . . . . . . . . . . . . 130

8.4 .3 Source context features . . . . . . . . . . . . . . . . 130

8.4 .4 Qualitative metrics . . . . . . . . . . . . . . 130

8.4 .5 More applications . . . . . . . . . . . . . . 131

\begin{tabular}{ll}
\hline Bibliography & 133
\end{tabular}

\begin{tabular}{ll}
\hline Appendix & 153
\end{tabular}

A. Gradient derivation . . . . . . . . . . . . . . . . . 153

B. Publications . . . . . . . . . . . . . . . . 156 


\section{List of Figures}

$2.1 \quad$ Inverted index. The example shows that term $t_{1}$ is contained in documents $d_{2}$ and $d_{27}$ with frequency 5 and 100 respectively. . . . . . . . 12

2.2 Documents and query represented in vector space. . . . . . . . . . . . 13

2.3 Framework for a cross-view task. . . . . . . . . . . . . . . . . . . . . 22

3.1 Architecture of a neuron . . . . . . . . . . . . . . . . . . 26

3.2 Multidimensional hidden layer neural network . . . . . . . . . . . . . 27

3.3 Sample architecture of a deep autoencoder. The binary and multinomial deep autoencoders are denoted as bDA and $\mathrm{rsDA}\left|\mathrm{Dim}_{i n}\right|$ is the dimensionality at the input layer. . . . . . . . . . . . . . . . 33

4.1 The architecture of the autoencoder (K-500-250-m) during (a) pretraining and (b) fine-tuning. After training, the abstract level representation of the given terms can be obtained as shown in (c). . . . . . 42

4.2 Contrastive divergence technique to pre-train RBM . . . . . . . . . . 43

4.3 System overview of training of XCNN model. . . . . . . . . . . . . . . 45

$4.4 \quad$ Embedding space before and after cross-lingual extension training. . . 46

4.5 Composition Model. . . . . . . . . . . . . . . . . . . . . . . . 47

$4.6 \quad$ Relevance backpropagation model for monolingual pre-initialisation of the latent space using monolingual relevance data. . . . . . . . . . . . 49

xvii 
4.7 Cross-lingual extension model. . . . . . . . . . . . . . . . . . . . 50

5.1 Average number of equivalents found in abstract space at similarity threshold $(\theta)$ (c.f. Section 4.1.4). . . . . . . . . . . . . . . . . . . . . 68

5.2 Impact of similarity threshold $(\theta)$ on retrieval performance. CCA $^{*}$ follows the ceiling X-axis range [0.999-0.99]. . . . . . . . . . . . . . 69

$5.3 \quad$ Snippet of mining lexicon projected in abstract space using CAE. . . . 70

6.1 Document-term matrix formulated from a parallel sentences corpus. . 80

6.2 Illustration of the proposed similarity feature to help choosing translation units. . . . . . . . . . . . . . . . . . . . . . . . . . . 102

6.3 Workflow of the system. . . . . . . . . . . . . . 103

7.1 Histogram of cosine similarity between test samples and their reconstructions for bDA and rsDA. . . . . . . . . . . . . . . . . . 115

7.2 Reconstruction error, SPI and SAI metrics when varying the bottleneck layer size from 100 to 10 are shown in (a), (b) and (c), respectively. 117

7.3 The percentage difference in slope of the SPI curve at each dimension. 120 


\section{List of Tables}

5.1 Classification of transliterated Hindi queries. Transliterated words are italicised. . . . . . . . . . . . . . . . . 60

5.2 The statistics of queries with query-categories in terms of the \% of unique queries and the $\%$ of total queries. . . . . . . . . . . . . . 61

5.3 Details of the dataset $\ldots \ldots \ldots$. . . . . . . . . . . . . . . 63

5.4 Example of query formulation for transliterated search. ${ }^{*}$ Note: $\$$ and , are added for readability. . . . . . . . . . . . . . . . . . . . . . . 64

5.5 The results of retrieval performance measured by MAP and MRR. Similarity threshold $\theta$ is tuned for best performance. . . . . . . . . . 67

5.6 The performance comparison of systems presented as $\mathrm{x} / \mathrm{y}$ where $\mathrm{x}$ denotes \% increase in MAP and y denotes $p$-value according to paired significance t-test. . . . . . . . . . . . . . . . . 67

5.7 Examples of the variants extracted using CAE with similarity threshold 0.96 (words beginning with! and ? mean "wrong" and "not sure" respectively). . . . . . . . . . . . . . . . 70

$6.1 \quad$ Statistics of PAN-PC-11 cross-language plagiarism detection partitions. 88 
6.2 ES-EN and DE-EN performance analysis in terms of $\mathrm{R} @ k$, where $k$ $=\{1,5,10,20\}$. Best results within each category are highlighted in bold-face. . . . . . . . . . . . . . . . . . . . . . . . . . 91

6.3 ES-EN and DE-EN performance analysis in terms of plagdet (Plag), precision (Prec), recall (Rec) and granularity (Gran). The best results within each category are highlighted in bold-face and $\dagger$ represents statistical significance, as measured by a paired t-test $(p$-value $<0.05)$. $\quad 93$

6.4 Results for the monolingual ad-hoc retrieval task measured in nDCG, MAP and MRR. . . . . . . . . . . . . . . . . . . . . . . . . . . 97

6.5 Results for the ad-hoc retrieval task measured in nDCG, MAP and MRR for title topic field. The best results are highlighted in bold-face and $\dagger$ represents statistical significance, as measured by a paired t-test

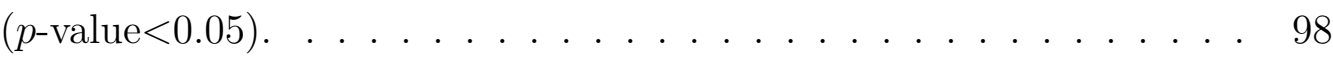

6.6 Results for the ad-hoc retrieval task measured in nDCG, MAP and MRR for title topic field considering only those queries for which more than $80 \%$ query-terms appear in the vocabulary. The best results are highlighted in bold-face and $\dagger$ represents statistical significance, as measured by a paired t-test $(p$-value $<0.05)$. . . . . . . . . . . . . . . 98

6.7 $\quad$ Results for the parallel sentence retrieval task measured in MRR. The best results are highlighted in bold-face and $\dagger$ represents statistical significance, as measured by a paired t-test $(p$-value<0.01). . . . . . . 101

6.8 BLEU scores for En2Es and En2Hi translation tasks. ${ }^{*}$ and ${ }^{\dagger}$ depicts statistical significance ( $p$-value $<0.05)$ wrt Baseline and LSA respectively. 104

6.9 Manual analysis of translation outputs. Adding the source context similarity feature allows for a more adequate lexical selection. . . . . 105

6.10 Probability values (conditional and posterior as standard features in a phrase-based system) for the word bands and two Spanish translations; and the word cry and two Hindi translations. . . . . . . . . . . . . . . 105 
7.1 The performance of bDA and rsDA in terms of different metrics. RC, SPI and SAI denote reconstruction error, structure preservation index and similarity accumulation index while $p t$ and $b p$ denote if the model is only pre-trained or fine-tuned after pre-training, respectively. .. . 113

7.2 Vocabulary sizes of the Bible dataset. . . . . . . . . . . . . . . . . . 118

7.3 The word trigram perplexities for each language considering tokens (PPL-T) and stems (PPL-S) along with critical bottleneck dimensionality. . . . . . . . . . . . . . . . . . . . . 121

7.4 The correlation between critical dimensionality for a language and its word trigram perplexity. The $p$-value represents the two-tailed TTest values. ${ }^{*}$ denotes the statistical significance $p<0.05$. . . . . . . . . . 122 
xxii

LIST OF TABLES 
casedect 1

\section{Introduction}

It has gone beyond the capabilities of a user to keep up with the information in the age of world wide web (WWW). Information sources on the web are heterogeneous such as web documents, tweets, news streams, videos, maps, images etc. Especially to search over these various sources of information, users typically rely on search technologies. The popularity of web search engines like Google $e^{1}$ and Bing ${ }^{2}$ is a clear example of this trend. Information retrieval is a field which studies search technologies.

With the advent of new input methods, multi-lingual content is increasing much faster on the web. This also increases the search traffic for multi-lingual content (Lazarinis et al., 2007; Hollink et al., 2004). Cross-language information retrieval (CLIR) approaches caters the task of information need in a language different to that of the collection. CLIR techniques have found many applications in real-world problems such as multilingual ad-hoc retrieval (Braschler et al., 1998, 1999; Braschler, 2004), cross-language plagiarism detection (Potthast et al., 2011; Barrón-Cedeño

\footnotetext{
${ }^{1}$ https://google.com
}

${ }^{2}$ https://bing.com 
et al. 2013; Franco-Salvador et al., 2013), parallel data compilation to aid statistical machine translation (Adafre and de Rijke, 2006; Fung and Cheung, 2004; Munteanu and Marcu, 2005) etc.

A large number of languages, including Arabic, Russian, and most of the South and South East Asian languages, are written using indigenous scripts. However, due to various socio-cultural and technological reasons, often the websites and the user generated content in these languages, such as tweets and blogs, are written using Roman script (Ahmed et al. 2011). Such content creates a monolingual or multi-lingual space with more than one scripts which we refer to as the mixed-script space.

Paired instances of data which provide the same information about each datum in different modalities are referred to as cross-view data. For example, parallel sentences are two different views of a sentence in different languages. A word and its transliteration 3 can be seen as two different views of the same word in different scripts. In cross-view tasks, instances of different views are not directly comparable. Under this terminology, CLIR and mixed-script information retrieval (MSIR) can be seen as cross-view retrieval tasks. Broadly, there are two approaches to cross-view tasks: $(i)$ translation; and ( $i$ ) cross-view projection. In translation approaches, oneview is translated into the other view using a translation model and the retrieval is carried using the other view. While, in cross-view projection approaches, data in both views are projected to an abstract common space using dimensionality reduction techniques, where they can be compared. Such representation is also referred to as embeddings. Though translation based approaches provide very rich representation of the data, such approaches are mainly devised for actual translation task such as machine translation (MT) of text from one language to the other. On the other hand, the projection methods provide a representation which may not be interpreted clearly, but provide more flexibility in obtaining representation pertaining to a particular task. For example, it is straight-forward to induce an objective function

\footnotetext{
${ }^{3}$ The process of phonetically representing the words of a language in a non-native script is called transliteration (Knight and Graehl, 1998).
} 
directly related to the task at hand in the learning mechanism e.g. increase cosine similarity between similar documents for a retrieval task. In this dissertation, we explore the cross-view embedding models for cross-view retrieval tasks.

The remaining of this chapter is structured as follows. First, the main contributions of the dissertation are listed in Section 1.1. We formulate main research questions investigated in this dissertation in Section 1.2. We present the outline of the thesis along with a brief chapter-wise description of the content in Section 1.3 .

\subsection{Contributions}

The contributions of this dissertation are many-fold. For the first time, we introduce the concept of MSIR formally (Gupta et al., 2014, Gupta, 2014). We also present the deep learning based cross-view models which provide the state-of-the-art performance for modelling mixed-script term equivalents for MSIR. The embedding based cross-view models: ( $i$ ) cross-view autoencoder; and (ii) external-data compositional neural network (XCNN) provide state-of-the-art performance for many cross-view tasks such as cross-language ad-hoc IR, parallel sentence retrieval, cross-language plagiarism detection, source context features for machine translation and mixedscript IR. This dissertation also provides insightful information about the structural properties of the autoencoder architecture, which helps to analyse the training process in a more intuitive way. We provide more details on each of this contributions in Sections 1.1.1, 1.1.2 and 1.1.3.

\subsubsection{Mixed-script information retrieval}

Information retrieval in the mixed-script space, which can be termed as mixed-script IR, is challenging because queries written in either the native or the Roman scripts need to be matched to the documents written in both the scripts. Transliteration, especially into Roman script, is used abundantly on the web not only for documents, but also for user queries that intend to search for these documents. Since there 
are no standard ways of spelling a word in certain non-native scripts, transliterated content almost always features extensive spelling variations; typically a native term can be transliterated into Roman script in very many ways (Ahmed et al., 2011). For example, the word pahala ("first" in Hindi and many other Indian languages) can be written in Roman script as pahalaa, pehla, pahila, pehlaa, pehala, pehalaa, pahela, pahlaa and so on.

This phenomenon poses a non-trivial term matching problem for search engines to match the native-script or Roman-transliterated query with the documents in multiple scripts taking into account the spelling variations. The problem of MSIR, although prevalent in web search for users of many languages around the world, has received very little attention till date. There have been several studies on spelling variation in queries and documents written in a single (native) script (Hall and Dowling, 1980; Zobel and Dart, 1996, French et al., 1997) as well as transliteration of named entities (NEs) in IR (Chen et al., 1998; Udupa and Khapra, 2010b; Zhou et al. 2012). However, as we shall see in Chapter 5, MSIR presents challenges that the current approaches for solving mono-script spelling variation and NE transliteration in IR are unable to address adequately, especially because most of the transliterated queries (and documents) belong to the long tail of online search activity, and hence do not have enough clickthrough evidence to rely on.

In this dissertation, we formally introduce the problem of MSIR and related research challenges (Gupta et al., 2014; Gupta, 2014). In order to estimate the prevalence of transliterated queries, analyses from a large query log of Bing consisting of 13 billion queries issued from India is also presented. As many as $6 \%$ of the unique queries have one or more Hindi words transliterated into Roman scripts, of which only $28 \%$ queries are pure NEs (people, location and organization). On the other hand, $27 \%$ of the queries belong to the entertainment domain (names of movies, song titles, parts of lyrics, dialogues, etc.), which provide complex examples of transliterated queries. Hindi music is also one of the most searched items in Indiaf and thus a

\begin{tabular}{cccc}
\hline 4Zitgeist 2010: & India & http://ww .google.com/intl/en \\
/press/zeitgeist2010/regions/in.html
\end{tabular}


practical case for MSIR.

\subsubsection{Cross-view models}

We present a principled solution to handle the mixed-script term matching and

spelling variation where the terms across the scripts are modelled jointly Gupta et al., 2014). We model the mixed-script features jointly in a deep learning architecture in such a way that they can be compared in a low-dimensional abstract space. The proposed method can find the equivalents of a given query term across the scripts; the original query is then expanded using the found equivalents. Through rigorous experiments on MSIR for Hindi film lyrics, we further establish that the proposed method achieves significantly better results compared to all the competitive baselines with 12\% increase in MRR and 29\% increase in MAP over the best performing baseline.

Although cross-view autoencoder provides a good way to model mix-script equivalents, it has some limitations in modelling text. In contrast to the most of the existing models which rely only on the comparable/parallel data, our model (externaldata compositional neural network - XCNN) takes the external relevance signals such as pseudo-relevance data to initialise the space monolingually and then, with the use of a small amount of parallel data, adjusts the parameters for different languages (Gupta et al. 2016a). There are a few approaches which go beyond the use of only parallel data. The framework also allows the use of clickthrough data, if available, instead of pseudo-relevance data. Our model, differently from other models, optimises an objective function that is directly related to an evaluation metric for retrieval tasks such as cosine similarity. These two properties prove crucial for XCNN to outperform existing techniques in the cross-language IR setting. We test XCNN on different tasks of CLIR and it attains the best performance in comparison to a number of strong baselines including machine translation based models. 


\subsubsection{Critical bottleneck dimensionality}

Although deep learning techniques are in vogue, there still exist some important open questions. In most of the studies involving the use of these techniques for dimensionality reduction, the qualitative analysis of projections is never presented. Typically, the reliability of the autoencoder is estimated based on its reconstruction capability.

The dissertation proposes a novel framework for evaluating the quality of the dimensionality reduction task based on the merits of the application under consideration: the representation of text data in low dimensional spaces. Concretely, the framework is comprised of two metrics, structure preservation index (SPI) and similarity accumulation index (SAI), which capture two different aspects of the autoencoder's reconstruction capability (Gupta et al., 2016c). More specifically, these two metrics focus on assessing the structural distortion and the similarities among the reconstructed vectors, respectively. In this way, the framework gives better insight of the autoencoder performance allowing for conducting better error analysis and evaluation. With the help of these metrics, we also define the concept of critical bottleneck dimensionality which refers to the adequate size of the bottleneck layer of an autoencoder.

We also conduct a comparative evaluation across different languages of the dimensionality reduction capabilities of deep autoencoders. With this empirical evaluation we aim at shedding some light on the adequacy of reducing different languages to a common bottleneck dimension, which is a common practice in the field.

\section{$1.2 \quad$ Research Questions (RQ)}

Here, we list the research questions that are investigated in this dissertation.

RQ1 To what extent mixed-script IR is prevalent in web-search and what is the best way to model terms for it? [Chapter 5 
RQ2 How effective is text representation obtained using external data composition neural network for cross-language IR applications? [Chapter 6]

RQ3 How cross-view autoencoder is useful for lexical selection issue in machine translation? [Chapter 6]

RQ4 How should the number of dimensions in the lowest-dimensional representation of a deep neural network autoencoder be chosen? [Chapter 7 ]

\subsection{Outline of the dissertation}

The dissertation is organised into four broad blocks: $(i)$ we first introduce the background of the main topics of the thesis (Chapters $2 \& 3$ ); (ii) we present the theoratical models proposed in this dissertation (Chapter 4); (iii) we present the evalu-

ation results and analyses for the proposed models on cross-view tasks (Chapters 5 \& 6); (iv) finally, we present analyses on structural properties for a proposed model (Chapter 7). More details about the organisation of each chapter is presented below.

Chapter 2 discusses the theoretical background on information retrieval and dimensionality reduction. It also presents the main challenges and current state-ofthe-art around these topics.

Chapter 3 presents necessary background on neural networks, Boltzmann machines, autoencoders and the optimisation methods to understand the technical details of the proposed models.

Chapter 4 presents the main technical contributions of the dissertation and explains the necessary details of the proposed models. We present the proposed crossview autoencoder based framework to model mixed-script terms and the details of the external-data compositional neural network (XCNN) model.

Chapter 5 presents the details of the mixed-script information retrieval. We first formally define the problem of mixed-script information retrieval with research challenges. We further analyse the query logs of the Bing search engine to understand 
better the mixed-script queries and their distributions. Finally, we present extensive performance evaluation of the proposed model based on cross-view autoencoder on a standard collection along with other state-of-the-art methods and present insightful analyses.

Chapter 6 presents the evaluation results of the proposed models on cross-language information retrieval tasks such as CL ad-hoc retrieval, parallel sentence retrieval, cross-language plagiarism detection and source context modelling for machine translation. For each application, we first give the description of the problem statement followed by the details of the existing methods. Finally, the comparative evaluation on standard benchmack collections is presented with necessary analysis.

In Chapter 7, we present two metrics, structure preservation index and similarity accumulation index. First, we define these metrics and present the underlying intuition capturing the different aspects of the autoencoder's reconstruction capabilities. With the help of these metrics we define the notion of critical bottleneck dimensionality for the autoencoder. Finally, through the multilingual analysis on a parallel data we show that different languages have different critical bottleneck dimensionalities, which happens to be closely associated with the language grammatical complexities, measured in terms of n-gram perplexities.

Finally in Chapter 8, we draw the conclusions from the dissertation, discuss limitations and outline the future work. 
Chapter 2

\section{Theoretical background}

This chapter aims at providing the necessary technical background for the work conducted in this dissertation as well as its related work in the literature. Being the central part of the dissertation, and in the interest of a wider audience, we first introduce the concepts related to information retrieval (IR) and dimensionality reduction in Section 2.1 and 2.2 respectively. Later we move to specific and related topics such as IR across languages and scripts in Section 2.3 and 2.4 respectively, which discuss the literature survey on the main topics of the dissertation. Finally, in Section 2.5, we introduce the terminology and framework of cross-view models.

\subsection{Information retrieval (IR)}

The formal definition of information retrieval as per Manning et al. (2008) is given below:

"Information retrieval is finding material (usually documents) of an unstructured nature (usually text) that satisfies an information need from within large collections 
(usually stored on computers)."

The reference to term "material" is quite broad and covers a lot of modalities and applications such as documents, images, videos, tweets, books, emails, music etc. In this work, we limit ourselves to text data. There are three different levels of information retrieval, based on the scale the retrieval is happening 1 .

1. Web search: The collection comprise of the web content which is enormous. A few examples are Google, Bing etc.

2. Personal search: In this case, the collection is typically a set of files on a personal computer of the user. For example, file search in operating systems.

3. Enterprise search: In this case, the collection comprises of a set of documents from a particular organisation or company. It can be domain specific. For example, intranet search.

Usually, the information need is described by the user in the form of query typically a few words long. Although it is assumed that the user always succeeds in describing the information need by means of a query, many times this is not necessarily true. There has been research in assisting users to formulate the query. The query auto-completion is a strong example of such methods (Bast and Weber, 2006: Bar-Yossef and Kraus, 2011). Lately, research has also focused on session-based models, which try to satisfy user information need by considering all the user input queries in the same search session (Raman et al., 2013; Carterette et al., 2014). The IR system satisfies the user information need in form of a ranklist of offerings.

\subsubsection{Vector space model}

In vector space model (VSM), documents and queries are represented as vectors in a high-dimensional space where each dimension is a term of the document (Salton

\begin{tabular}{l}
\hline${ }^{1}$ This categorisation is only meant for comparing the scale and it should not be confused as a \\
categorisation of IR applications.
\end{tabular}


et al. 1983). Let a document $d$ be represented as $\vec{d}=\left\{t w_{1}, t w_{2}, \cdots, t w_{n}\right\}$, where $t w_{i}$ denotes the term weight of $i^{t h}$ term in the document. All the terms present in the document will have a non-zero entry in $\vec{d}$. There are multiple ways to calculate these term-weights (Singhal et al. , 1996), one popular way is term frequency-inverse document frequency (TF-IDF).

$$
t w_{i}^{d}=t f_{i}^{d} * i d f_{i}
$$

where,

$$
\begin{gathered}
t f_{i}^{d}=\text { frequency of } i^{t h} \text { term in document } d \\
i d f_{i}=\log \left(\frac{\text { total number of documents in collection }}{\text { number of documents term i appears in }}\right)
\end{gathered}
$$

The $t f$ term captures the importance of the term in the document while $i d f$ captures the rareness of the term in the collection. In VSM, the definition of term is abstract as it can be single word, phrase or characters based on the application in hand. The total number of unique terms in the collection defines the dimensionality of the vector space.

\subsubsection{Indexing and retrieval}

The vectors in VSM are usually sparse and storing the complete vector is not always possible. Hence, only the non-zero entries are stored. It should also be noted that not all documents are needed to be processed for a particular query. One way to optimise the complete traversal is to process only those documents which contain at least one query term. The frequency statistics required for models like TF-IDF are stored in a data structure called an inverted index. An inverted index has two main components, the terms present in the index - term-index; and the list of documents they appear in with necessary statistics - posting-list. An example of inverted index is depicted in Fig. 2.1. Here, the term-index is a simple array which has time complexity of $O(n)$ 


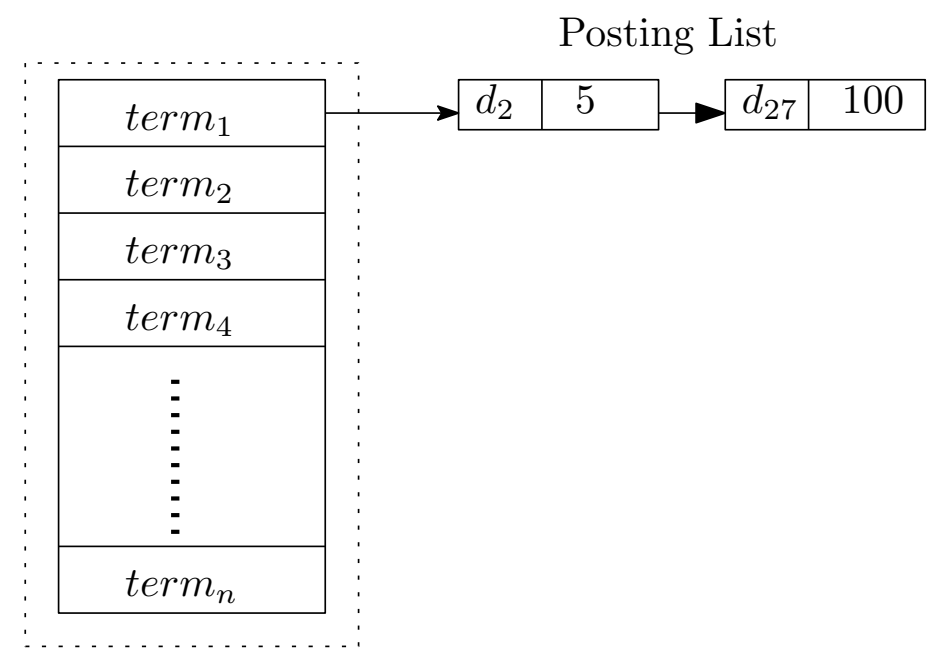

Term Index

Figure 2.1: Inverted index. The example shows that term $t_{1}$ is contained in documents $d_{2}$ and $d_{27}$ with frequency 5 and 100 respectively.

while the posting-lists are storing the frequency of the terms in the corresponding documents. There are many variants of the inverted index, mainly attributed by their (search) time and space complexity constraints (Zobel and Moffat, 2006).

At the time of retrieval, the similarity between a query and documents (both represented in the vector space) are estimated by means of the angular distance between them as shown in Fig. 2.2. The cosine angle provides a similarity metric which is estimated as described in Eq. 2.1.

$$
\operatorname{sim}(\vec{d}, \vec{q})=\cos (\vec{d}, \vec{q})=\frac{\vec{d} \cdot \vec{q}}{\|\vec{d}\|\|\vec{q}\|}
$$

\subsubsection{Evaluation}

Evaluation for IR systems has been a very active area of research because of the empirical nature of the field. There have been many evaluation metrics proposed which capture different aspects of the system performance (Manning et al., 2008). There are two types of performance evaluations related to effectiveness and efficiency. 


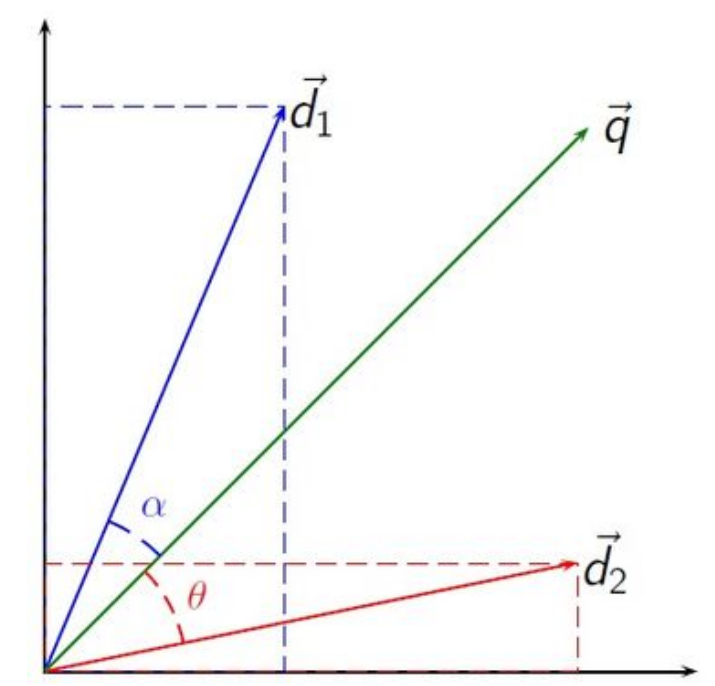

Figure 2.2: Documents and query represented in vector space.

The latter deals with the issues such as query latency and memory requirements. While the former, the effectiveness, attracts larger research attention. It mainly dwells around the concept of relevance. Although, relevance is a quite subjective and abstract concept, it is usually captured by the manual relevance judgements (qrels). Human judges are presented with a set of queries, document collection and corresponding relevance judgements and they assign a binary label to the document: relevant or non-relevant. The label relevant is assigned if the document satisfies the information need expressed by the query.

Precision captures the ratio of the relevant documents among the retrieved documents and Recall captures the ratio of retrieved relevant documents among all the relevant documents available in the collection. Most of the IR systems try to find a trade-off between Precision and Recall with an extra bias towards either of them, depending on the specific application. For example, web search engines are more keen on Precision, while medicine or legal aspects related system care more for Recall. $F_{\beta}$-measure is a popular way of combining Precision and Recall, where $\beta$ decides the bias towards precision or recall. $F_{1}$ gives equal weight to precision and recall while $\mathrm{F}_{2}$ gives higher weight to recall and $\mathrm{F}_{0} .5$ gives higher weight to precision. 


$$
F_{\beta}=\left(1+\beta^{2}\right) \cdot \frac{\text { precision } \times \text { recall }}{\left(\beta^{2} \cdot \text { precision }\right)+\text { recall }}
$$

Average precision (AP) calculates precision at every recall point. AP provides a way to estimate the quality of the ranklist. Sometimes, the relevance is labeled in higher levels (graded-levels) to quantify better than binary. As the discussed metrics so far work with binary relevance, we have used normalised discounted cumulative gain (NDCG) which uses graded relevance as a measure of the usefulness, or gain, from examining a document (Järvelin and Kekäläinen, 2002). Gain is calculated at each ranking position and accumulated over all positions with a discount element. The assumption is the relevant documents at lower position are less useful because they are quite likely will not be examined by the user. A typical discount function is $\frac{1}{\log _{2}(\mathrm{rank})}$. The cumulative gain $c g_{m}$ for a ranklist of size $m$ is calculated as:

$$
c g_{m}=\sum_{i=1}^{m} \operatorname{rel}\left(d_{i}\right)
$$

where, $\operatorname{rel}\left(d_{i}\right)$ is graded-relevance of document $d_{i}$. Adding the discount term, discounted cumulative gain $d c g_{m}$ becomes:

$$
d c g_{m}=\operatorname{rel}\left(d_{1}\right)+\sum_{i=2}^{m} \frac{\operatorname{rel}\left(d_{i}\right)}{\log _{2}(i)}
$$

$d c g_{m}$ is normalised by the ideal ranklist upto $m$ positions referred as $i d c g_{m}$ to obtain $n d c g_{m}$.

$$
n d c g_{m}=\frac{d c g_{m}}{i d c g_{m}}
$$

\subsubsection{Semantic term-relatedness}

Vector space models provide a way to compare documents and queries by means of keyword matching. However, such lexical matching can be inaccurate due to the fact that the relevance is often expressed by different vocabularies in documents and 
queries. One of the major hurdles in comparing text in VSM is to deal with problems like synonymy and polysemy. Usually, in vector space, the documents are composed of thousands of dimensions resulting in many meaningful associations between terms being shadowed by large dimensions. There are models which try to handle this problem in the vector space e.g. pseudo relevance feedback (PRF) (Rocchio, 1971; Manning et al., 2008) and explicit semantic analysis (ESA) (Xu and Croft, 1996 ; Gabrilovich and Markovitch, 2007, Anderka and Stein, 2009). PRF obtains top $m$ terms from top $n$ documents and adds them to the original query and the expanded query is used for the retrieval. ESA based approaches leverage on an external collection, such as Wikipedia, which is referred to as knowledge base. In ESA each word is represented in the retrieval collection by its corresponding vector of document scores in the knowledge base. Then, relatedness between two terms is calculated by the cosine similarity between the corresponding vectors. Word sense disambiguation for information retrieval has also been an active area of research (Sanderson, 1994; Liu et al., 2005).

\subsection{Dimensionality reduction}

A formal definition of dimensionality reduction as per Burges (2010) is given as:

"Dimensionality reduction is the mapping of data to a lower dimensional space such that uninformative variance in the data is discarded, or such that a subspace in which the data lives is detected."

Basically, it is a process of reducing the number of variables under consideration. Dimensionality reduction techniques are widely popular in learning representation of data in different modalities such as text, image, audio, video, etc Fodor, 2002, Burges, 2010). In this work we would focus concretely on approaches related to text data.

Dimensionality reduction techniques can be achieved in two ways: (i) feature selection; and (ii) feature extraction. The feature selection methods reduces the di- 
mensionality by selecting a subset of features from the set of original features. The feature selection methods of type filter, as defined in Guyon and Elisseeff (2003), computes the score of each feature as a preprocessing step and the subset of features are selected based on the scores assigned. In contrast to filter methods, wrapper methods use the learning algorithms to assign scores to the features. Feature selection techniques are widely used in machine learning based approaches, such as classification (Dash and Liu, 1997; Yang and Pedersen, 1997; Janecek et al., 2008) and ranking (Geng et al., 2007; Gupta and Rosso, 2012a).

On the other hand, the goal of the feature extraction based techniques is to transform high dimensional data $\left(\mathbb{R}^{n}\right)$ into a much lower dimension representation $\left(\mathbb{R}^{m}\right)$ pertaining the inherent structure of the original data where $m<<n$. Such low-dimensional space is commonly referred to as abstract space or latent space. One such widely used approach is latent semantic indexing (LSI), which extracts a low rank approximation of text data by means of singular value decomposition (SVD) (Deerwester et al. 1990).

Dimensionality reduction techniques can broadly be categorised in two classes: linear and non-linear. Usually, non-linear techniques can find more compact representations of the data compared to their linear counterparts Hinton and Salakhutdinov. 2006). If there exists statistical dependence among the principal components of PCA, or principal components have non-linear dependencies, PCA would require a larger dimensionality to properly represent the data when compared to non-linear techniques.

On the other hand, although non-linear projection methods such as multidimensional scaling (MDS) give a way to obtain much better representations for mono and cross-language similarity estimation; it is a transductive method (Cox and Cox 2000; Banchs and Kaltenbrunner, 2008). It means MDS does not provide an operator to project the unseen data into the target low dimensional space like the resulting projection matrix in the case of PCA.

Latent semantic models such as LSI are able to correspond queries and relev- 
ant documents at the semantic level where lexical matching often fails (Deerwester et al., 1990; Blei et al., 2003; Salakhutdinov and Hinton, 2009b; a; Platt et al., 2010; Huang et al. 2013). These latent semantic models represent the text in a dense low-dimensional semantic space, where semantically similar text fragments would be closer to each other despite the fragments do not share any term. The semantic representation is learned through the patterns of terms co-occurring in similar contexts. LSI extracts a low rank Gaussian approximation of a document-term matrix ${ }^{2}$ by means of singular value decomposition (SVD) (Deerwester et al., 1990). More advanced approaches like probabilistic latent semantic analysis (PLSA) and latent dirichlet allocation (LDA) observe the distribution of latent topics for the given documents (Hofmann, 1999; Blei et al., 2003).

Lately, dimensionality reduction techniques based on deep learning have become very popular, especially deep autoencoders (DA). Deep autoencoders can extract highly useful and compact features from the structural information of the data. Deep autoencoders have proven to be very effective in learning reduced space representations of the data for similarity estimation, i.e. similar documents tend to have similar abstract representations (Hinton and Salakhutdinov, 2006; Salakhutdinov and Hinton, 2009a). Deep-learning is inspired by biological studies, which state the brain has a deep architecture. Despite their high suitability to the task, deep learning did not find much audience because of convergence issues until Hinton and Salakhutdinov (2006) gave a way to initialise the network parameters in a good region for finding optimal solutions.

However, these models are trained to optimise an objective function which is only loosely related to the evaluation metric of the retrieval task. To overcome this limitation, a new family of latent semantic models have emerged that exploits the clickthrough data for semantic modelling Gao et al. (2010, 2011); Huang et al. (2013). These models take into account an explicit relevance signal in terms of the query and

\footnotetext{
${ }^{2}$ Such matrix is composed by the documents in the collection (rows) and all the unique terms in the collection (columns). Each entry in the matrix contains the weight of a particular term in a document e.g. term frequency.
} 
its clicked document.

\subsection{IR across languages}

Cross-language information retrieval (CLIR) is a special case of IR, where the query and the documents are in different languages. Due to the existing needs in different multi lingual scenarios, various CLIR applications became popular. Cross-language ad-hoc retrieval, cross-language plagiarism detection and parallel/comparable text discovery are examples of some popular and important problems.

In general, there are two ways to address the language mismatch between query and documents: $(i)$ translate either of them to the language of the other and perform monolingual IR; and ( $i$ ) obtain a language agnostic translingual space where both of them can be compared. The former takes the path of machine translation while the latter falls under the dimensionality reduction techniques ${ }^{3}$.

The translation approaches try to normalise language mismatch between query and documents using various resources such as bilingual dictionaries, multilingual thesaurus, multilingual semantic network etc. Machine translation systems leverage on a translation model (estimating segment-leve $]^{4}$ translation probabilities) that is combined with a target language model. The language model helps aligning the potential segment-level translations to form a valid sentence. Typically, machine translation based approaches for CLIR do not employ the full MT pipeline, instead they exploit the translation probabilities to formulate the translated query Gao et al., 2001; Ture and Lin, 2014). Moreover, the MT based approaches often employ lexical, syntactic and semantic linguistic analysis. Though such pipeline ensures the representation is rich, they are mainly deviced for the MT task and this representation may not be that helpful for the retrieval task.

Though the MT based language normalisation can be highly accurate, the re-

\footnotetext{
${ }^{3}$ Though one can use dimensionality reduction techniques on machine translated text, what we refer here is to obtain translingual representation using dimensionality reduction techniques.

${ }^{4}$ Including both single words and multi-word phrases.
} 
trieval suffers from the issues of VSM such as sparsity, synonymy and polysemy. Moreover, MT can be very slow, limiting its use on large training datasets (Platt) et al., 2010; Gupta et al. 2016b). Alternatively, the cross-language latent semantic models provide a way to model cross-language term associations in a latent space. Such models include LSA based cross-language latent semantic analysis (CL-LSA) (D11mais et al., 1997), in which a cross-language document-term matrix is constructed by concatenating the parallel data. Canonical correlation analysis (CCA) based methods find projections that maximise the correlation between the projected vectors of parallel data (Vinokourov et al. , 2002). Generative models, such as LDA, are used to represent bilingual data into hidden topical space (Mimno et al., 2009). Oriented principal component analysis (OPCA) introduces the noise covariance matrix and solves the generalised eigenvalue problem (Diamantaras and Kung, 1996; Platt et al., 2010). Deep bilingual autoencoders (BAE) are used to represent bilingual data in a low-dimensional joint space by optimising the reconstruction error (Lauly) et al., 2014, Chandar A. P. et al., 2014: Gupta et al., 2014). Siamese neural network based S2Net learns discriminatively the projection matrix from the pairs of related and unrelated documents (Yih et al. 2011). Except for the S2Net method, all these models derive cross-language representations in an unsupervised manner by optimising an objective function that is only loosely related to the evaluation metric for the retrieval task. Some of these models are reviewed in detail in Chapter 6. Another family of models for cross-language natural language processing applications require advanced syntactic information in the input, such as syntactic parse trees (Socher et al., 2012; Hermann and Blunsom, 2013). Similar models sometimes also require word-alignments during the training (Klementiev et al., 2012, Zou et al., 2013; Mikolov et al., 2013). Such requirements limit the use of these approaches to resource fortunate languages. 


\subsection{IR across scripts}

Although IR across scripts, which is referred to as mixed-script IR, has attained very little attention explicitly, many tangentially related problems like CLIR and transliteration for IR discuss some of the issues of MSIR. While languages like Chinese and Japanese use multiple scripts ( $\mathrm{Qu}$ et al., 2003), they may not illustrate the true complexity of the MSIR scenario described here because there are standard rules and preferences for script usage and well defined spellings rules. In Roman transliteration of Hindi, on the other hand, there are no standard rules, which leads to a large number of spelling variations for a single term. Furthermore, these texts are often mixed with English, which makes detection of transliterated text quite difficult.

CLIR typically involve translating queries from one language to another. However, it is often a reasonable choice to transliterate certain OOV words, especially NEs. There has been a large body of work that specifically targets the problem of named entity transliteration in CLIR.

However, training and testing transliteration systems requires data and, for Names Entities, data creation has been typically through mining text corpora. Shared tasks such as those conducted by NEWS 5 and FIRE[ ${ }^{6}$ have also been successful to an extent in both data sharing and bench-marking various machine transliteration techniques and systems.

In an analysis of the query logs for Greek web users, Efthimiadis et al. (2009) have shown that 90 percent of the queries are formulated using the Roman alphabet while only $8 \%$ use the Greek alphabet, and the reason for this (Efthimiadis, 2008) is that 1 in 3 Greek navigational queries fail due to the low level of indexing of the Greek web. Wang et al. (2008) employ a translation based method to classify non-English queries using an English taxonomy system. Though their method shows some promise, it is heavily dependent on the availability of translation systems for the language pairs in question. Ahmed et al. (2011) show that the problem of transliteration is

\footnotetext{
${ }^{5}$ http://translit.i2r.a-star.edu.sg/news2012/

${ }^{6}$ http://www.isical.ac.in/ fire/
} 
further challenging because of the fact that due to a lack of standardization in the way a local language is mapped to the Roman script, there is a large variation in spellings. In their work on query-suggestion for a Bollywood Song Search system Dua et al. (2011) also stress on the presence of valid variations in spelling Hindi words in Roman script. Related work by Gupta et al. (2012a) goes into the details of handling these variations while mining transliterated pairs from Bollywood song lyric data. Edit-distance based approaches have also been popular for the generation of such pairs; such as, for instance, English-Telugu (Sowmya and Varma, 2009) and Tamil-English (Janarthanam et al., 2008). Pal et al. (2008) propose a method for normalization of transliterated text that combines two techniques: (i) a stemmer based method that deletes commonly used suffixes (Oard et al., 2001) with rules for mapping variants to a single canonical form; (ii) a similar method that uses both stemming and grapheme-to-phoneme conversion is used by Raj and Maganti (2009) to develop a proof-of-concept for a multilingual search engine that supports 10 Indian languages. Thus, though there has been some interest in the past, especially with respect to handling variation and normalization of transliterated text, the challenge of IR in the mixed-script space is largely neglected.

For languages like Japanese, Chinese, Arabic and most Indian languages, the challenge of text input in native script has resulted in a proliferation of transliterated documents on the web. While the availability of more sophisticated and user-friendly input methods have helped resolving this for some of these languages (for example Japanese and Chinese), there is still a large number of languages for which the English keyboard (and hence the Roman script) remains the main input medium. Further, as a number of relevant documents are available in both the native script and its transliterated form, it also becomes important to deal with not only cross-language but mixed-script IR for such languages. Social media is another domain where the use of transliterated text is widespread. Here, text normalization is complicated further by the presence of SMS-like contractions, interjections and code-mixing (the switching between languages at phrase, word and morphological 
levels). As IR becomes more pervasive in social media, dealing with the complexities of transliteration will become more significant for a robust search engine.

\subsection{Cross-view models}

Retrieval in cross-view setting is central to this dissertation contribution. Many times the data is represented in two or more different instances, e.g. similar text in different languages (parallel text), words in different script (transliteration), data in different modalities (word and an image describing it or text and its audio representation). In the case two instances of data are available, the data is referred to as cross-view data, while in the case that more than two instances are available, it will be referred to as multi-view data. The proposed general framework for a cross-view task is depicted in Fig. 2.3. Retrieval in a cross-view setting is a task of retrieving similar data items given some input data from both types of instances.

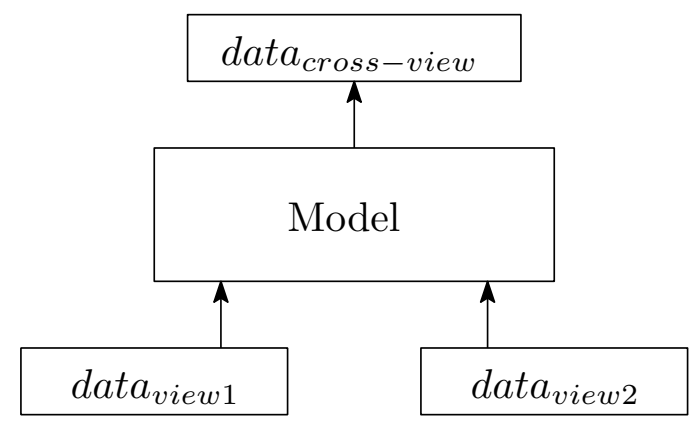

Figure 2.3: Framework for a cross-view task.

In this dissertation, we limit ourselves to text modality of the data but consider different applications of the cross-view setting such as:

1. cross-language ad-hoc retrieval: queries need to be matched with documents in different languages;

2. cross-language parallel sentence retrieval: sentences that are translations of each other in different languages need to be retrieved; 
3. cross-language plagiarism detection: plagiarised sections from the suspicious documents need to be identified from source documents in different languages;

4. mixed-script IR: queries need to be matched with documents written in the same language but different scripts.

The detailed description of these tasks and experimental framework are presented in Chapters 6 and 5 . 



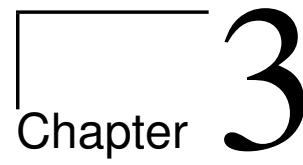

\section{Neural networks background}

This chapter covers the technical background needed to understand the proposed models in the thesis. First, we will provide an introduction to neural networks and restricted Boltzmann machines followed by autoencoders. We will also review the backpropagation algorithm and few relevant optimisation techniques.

\subsection{Neural networks}

Neural networks or artificial neural networks are a family of models inspired by biological neural networks. These models are used to approximate complex unknown functions that usually depend on a large number of inputs. The fundamental unit of a neural network is the artificial neuron. Fig. 3.1 shows the architecture of a single artificial neuron with input vector $v \in \mathbb{R}^{n}$, output $h \in \mathbb{R}^{m}$ and a parameter set of weights $w$ and bias $b$. Such basic processing unit, which is called an artificial neuron, encodes the input information into a real number output $h$, as shown in Eq. 3.1; where $g$ is a non-linear activation function. 


$$
h=g\left(\sum_{i} v_{i} * w_{i}+b\right)
$$

There are many variants of activation functions, being the most popular choices the sigmoid function (Eq. 3.2) and the hyperbolic tangent function (Eq. 3.3). It is important for an activation function to be differentiable, for reasons that will become clear in Sec. 3.5, where the backpropagation algorithm is discussed. It can be noticed that the sigmoid function maps any real number into the $[0,1]$ interval, while the hyperbolic tangent function does it into $[-1,1]$.

$$
\begin{gathered}
\operatorname{sigm}(x)=\frac{1}{1+e^{-x}} \\
\tanh (x)=\frac{e^{x}-e^{-x}}{e^{x}+e^{-x}}=\frac{1-e^{-2 x}}{1+e^{-2 x}}
\end{gathered}
$$

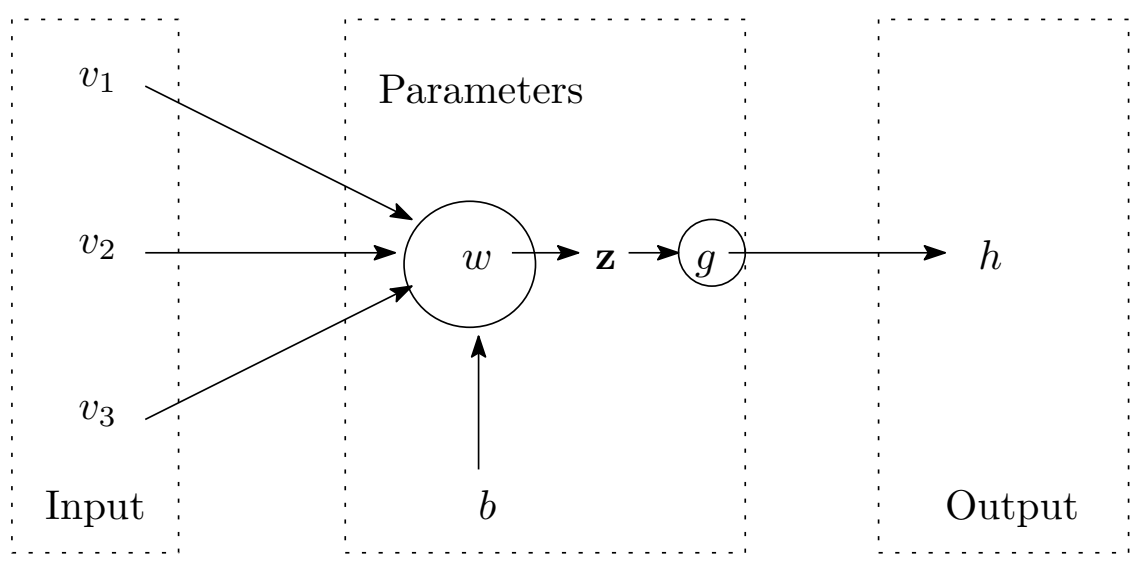

Figure 3.1: Architecture of a neuron

In practice, artificial neurons can be spatially arranged into layers to create a multi-dimensional processing arrays. These layers can be also combined in sequences to create a multi-layer processing structure. The input and output layers are generally referred to as visible layers and the all the intermediate layers are typically referred to as hidden layers. A simple example of multi-dimensional multi-layer 
neural network is shown in Fig. 3.2 .

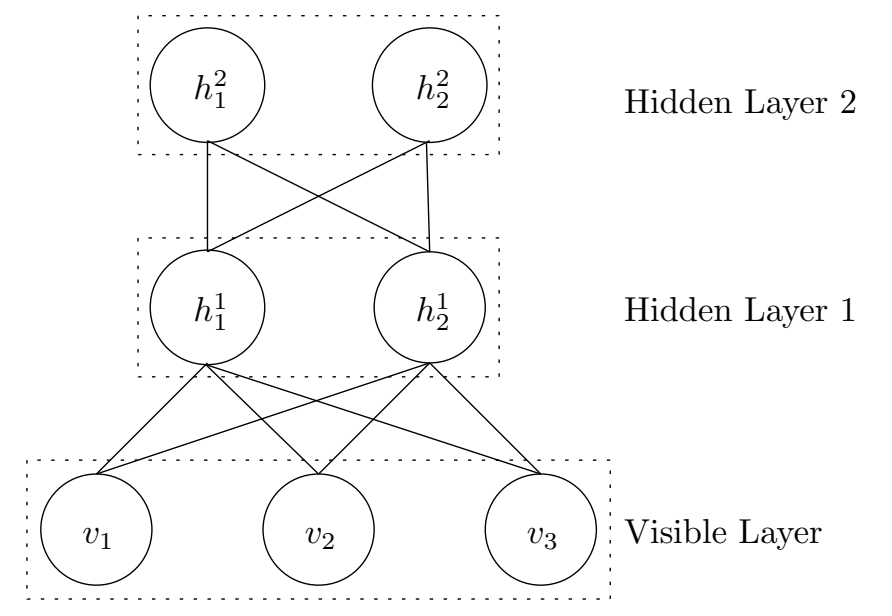

Figure 3.2: Multidimensional hidden layer neural network

For the neural network represented in Fig. 3.2, hidden neuron activities can be computed as shown in Eq. 3.4. Such network is also referred to as a feedforward neural network,

$$
\begin{aligned}
& \mathbf{z}^{(1)}=W^{(1)} * \mathbf{v}+\mathbf{b}^{(1)} \\
& \mathbf{h}^{(1)}=g\left(\mathbf{z}^{(1)}\right) \\
& \mathbf{z}^{(2)}=W^{(2)} * \mathbf{h}^{(1)}+\mathbf{b}^{(2)} \\
& \mathbf{h}^{(2)}=g\left(\mathbf{z}^{(2)}\right)
\end{aligned}
$$

where $\mathbf{h}^{(k)}$ represents the output of the $k^{\text {th }}$ hidden layer, $W^{(k)}$ is weight matrix for layer $k$ with $w_{i j}$ representing the weighting factor between unit $i$ in the previous layer and hidden unit $j$ in the current layer, $\mathbf{b}^{(k)}$ is the bias vector for layer $k$ and the activation function $g$ is applied to every neuron in the hidden layers.

The output layer of a neural network represents the solution space of the problem addressed by the network. In case of a binary classification problem, the output layer can be a single neuron with the sigmoid activation function. The output of such 
neuron can be interpreted as the probability $p$ for the corresponding input datapoint to belong to a certain class and $(1-p)$ as the probability to not belong. In case of a multi-class classification problem, the output layer is usually of size equal to the number of classes and, often, the softmax normalization function is applied at output layer. The softmax function allows the output layer of the network to model a probability distribution in which each neuron represents the probability of one of the considered classes. The softmax normalization function is shown in Eq. 3.5. For non-classification tasks such as regression, representation learning or projection, the output layer represents a multi-dimensional continuous space in which the input data is projected.

$$
\operatorname{softmax}\left(h_{j}\right)=\frac{e^{h_{j}}}{\sum_{k=1}^{K} e^{h_{k}}}, \text { for } j=1,2, . ., K
$$

\subsection{Restricted Boltzmann machines (RBM)}

Restricted Boltzmann machines have been used as generative models for many different types of data including images (Hinton and Salakhutdinov, 2006), speech (Mohamed et al., 2012), documents (Salakhutdinov and Hinton, 2009b), and user ratings (Salakhutdinov et al., 2007). A restricted Boltzmann machine is a two-layer bipartite network with a visible layer $(\mathbf{v})$ and a hidden layer $(\mathbf{h})$. Both layers are connected through symmetric weights $(\mathbf{w})$. In this kind of models the hidden units play the role of latent variables. Depending on the type of input data, two different variants of the visible layer can be selected: binary or multinomial. In case of binary RBM the visible layer is stochastic binary layer which accepts data in binary form. While, in case of multinomial RBM, visible layer is multinomial to accept data which follows multinomial distribution. The multinomial RBM is based on the replicated softmax model (RSM) (Salakhutdinov and Hinton, 2009b). Following, we present more details on the two RBM. 


\subsubsection{Stochastic binary RBM}

In a stochastic binary RBMs both, visible and hidden, layers are stochastic binary units with sigmoid non-linearity. Let visible units $\mathbf{v} \in\{0,1\}^{n}$ be binary input variables and hidden units $\mathbf{h} \in\{0,1\}^{m}$ be hidden latent variables. Energy-based models have an energy value associated to each configuration of the variables (i.e., parameters) of of the model. Parameters updated through the learning algorithm modify this energy and usually, the desirable parameters have low energy. RBMs are commonly explained using energy-based models (LeCun et al., 2006). The energy of the state $\{\mathbf{v}, \mathbf{h}\}$ is defined as follows:

$$
E(\mathbf{v}, \mathbf{h})=-\sum_{i=1}^{n} a_{i} v_{i}-\sum_{j=1}^{m} b_{j} h_{j}-\sum_{i, j} v_{i} h_{j} w_{i j}
$$

where $v_{i}, h_{j}$ are the binary states of visible unit $i$ and hidden unit $j, a_{i}, b_{j}$ are their biases and $w_{i j}$ is the weight between them.

Then, it is possible to obtain visible and hidden activities in both directions as shown below,

$$
\begin{aligned}
& p\left(v_{i}=1 \mid \mathbf{h}\right)=g\left(a_{i}+\sum_{j} h_{j} w_{i j}\right) \\
& p\left(h_{j}=1 \mid \mathbf{v}\right)=g\left(b_{j}+\sum_{i} v_{i} w_{i j}\right)
\end{aligned}
$$

where $g(x)=1 /(1+\exp (-x))$ is the logistic sigmoid function.

\subsubsection{Multinomial RBM}

The multinomial RBM is based on the Replicated Softmax model proposed by Salakhutdinov and Hinton (2009b).

Let $\mathbf{v} \in\{1, \ldots, K\}^{n}$, where $K$ is the number of trials parameter of multinomial distribution, $n$ is the input dimensionality and let $\mathbf{h} \in\{0,1\}^{m}$ be stochastic binary hidden latent variables. Considering an input with $K$ trials, the energy of the state 
$\{\mathbf{v}, \mathbf{h}\}$ is defined as:

$$
E(\mathbf{v}, \mathbf{h})=-\sum_{i=1}^{n} \hat{v}^{k} a^{k}-D \sum_{j=1}^{m} b_{j} h_{j}-\sum_{k, j} w_{i j}^{k} h_{j} \hat{v}^{k}
$$

where $v_{i}^{k}$ denotes the count $(k)$ data for the $i^{\text {th }}$ term.

In the RSM, the visible layer implements a softmax normalizing function. The resulting multinomial visible units represent the probability distribution of the wordcounts. In multinomial RBM, the visible and hidden units are updated as shown below,

$$
\begin{gathered}
p\left(v_{i}^{k}=1 \mid \mathbf{h}\right)=\frac{\exp \left(b_{i}^{k}+\sum_{j} h_{j} W_{i j}^{k}\right)}{\sum_{q=1}^{K} \exp \left(b_{i}^{q}+\sum_{j} h_{j} W_{i j}^{q}\right)} \\
p\left(h_{j}=1 \mid \mathbf{V}\right)=\sigma\left(a_{j}+\sum_{i=1}^{D} \sum_{k=1}^{K} v_{i}^{k} W_{i j}^{k}\right)
\end{gathered}
$$

\subsection{Text representation}

Text can be represented in vector form, popularly referred as bag-of-words. All possible words from the corpus constitute a vocabulary of unique words. These words in vocabulary form a high-dimensional vector space where each word refers to a dimension. A text can be represented as either a binary vector where non-zero dimension denotes existance of that word in the text or a count vector where nonzero dimension denotes the count of that word in the text. It is a common practice to remove stopwords from the vocabulary and apply stemming. 


\subsection{Autoencoder}

The autoencoder is a neural network architecture that approximates the identity function by replicating its input as its output. The input and output dimensions of the network are the same $(n)$. The autoencoder approximates the identity function in two steps: ( $i$ ) coding (reduction); and (ii) decoding (reconstruction). The coding step maps the input $x \in \mathbb{R}^{n}$ into an intermediate representation $y \in \mathbb{R}^{m}$ where $m<n$. The mapping can be seen as a function $y=g(x)$ with $g: \mathbb{R}^{n} \rightarrow \mathbb{R}^{m}$. On the other hand, the decoding step maps the intermediate representation $y$ into the output $\hat{x} \in \mathbb{R}^{n}$ in such a way that $\hat{x} \approx x$. The decoding step can be seen as a function $\hat{x}=f(y)$ with $f: \mathbb{R}^{m} \rightarrow \mathbb{R}^{n}$. The two-step autoencoder can be seen as the composition of an encoding function $g(x)$ and a decoding function $f(x)$, which approximates the identity function: $f(g(x)) \approx x$ using reconstruction error:

$$
\text { reconstruction error }=\frac{1}{2}\|f(g(x))-x\|^{2}
$$

In an autoencoder, the visible layer corresponds to the input $x$ and the hidden layer corresponds to the intermediate representation $y$. Autoencoders can have a single hidden layer or multiple hidden layers. If there is only one single hidden layer, the optimal solution remains the PCA projection (Bourlard and Kamp, 1988). In order to overcome some of the PCA limitations, a common practice is to stack multiple RBMs, constituting what is called a deep architecture. Those deep architectures have been proven to produce highly non-linear and powerful reduced space representation (Hinton and Salakhutdinov, 2006). Autoencoders made up from multiple RBMs are referred to as deep autoencoders. Both of the considered models differ in the way they model the text data. While the binary deep autoencoder (bDA) models the presence of the term into the document (binary), the multinomial deep autoencoder (rsDA) directly models the count of the term (i.e., term frequency) in the document. An example of deep architecture is shown in Fig. 3.3. 


\subsection{Backpropagation}

Backpropagation is an efficient method to train a multi-layered feedforward neural network (Rumelhart et al., 1986). It is typically used in conjunction with an optimisation method, such as gradient descent, which is discussed in more detail in Sec. 3.6. In backpropagation the gradient of a loss function is calculated with respect to the network parameters: weights and biases. The information of the gradient is recursively used to update the parameters in such a way that the specified loss function is reduced at each iteration step. Backpropagation is a supervised method because it needs to know the correct output in order to compute the loss function. An important condition for the use of backpropagation is that all transfer functions at each layer should be differentiable, otherwise the gradient calculation is not possible. Both, the errors calculated at the output layer and the corresponding gradient are propagated backwards through the entire network, hence the name of "backpropagation".

An important step in neural network training is the definition of the loss function $J(\theta)$, where $\theta$ represents the set of network parameters. One popular choice for loss function is the mean squared error (MSE), which is defined as $J(\theta)=\frac{1}{2}(y-h)^{2}$, where $y$ is label and $h$ is neural network output. Using the terminology introduced in Fig. 3.1 and considering a neural network with 2-hidden layers with sigmoid activation function and one single output, the gradient computation at the output layer is shown in Eq. 3.13 . 


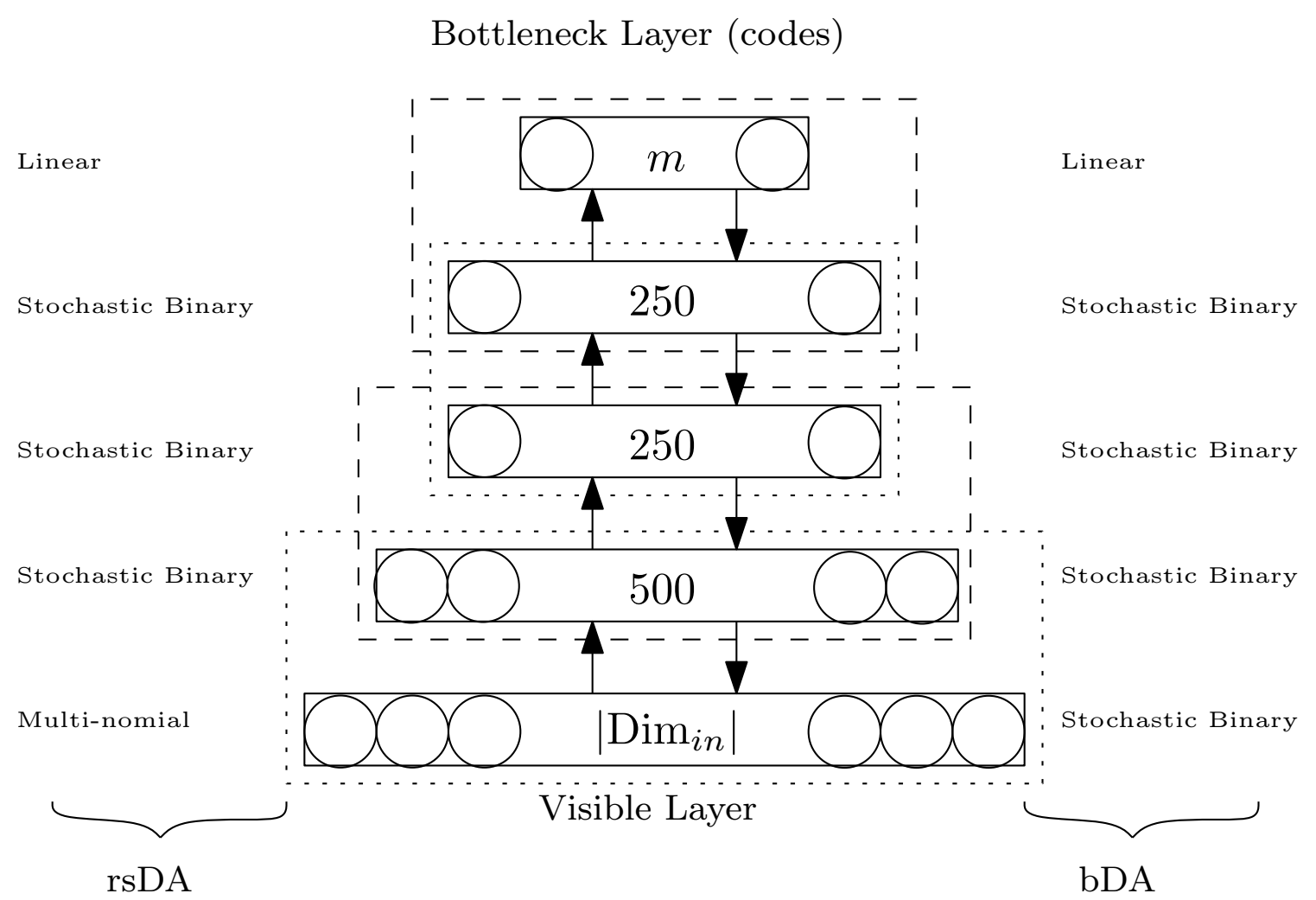

Figure 3.3: Sample architecture of a deep autoencoder. The binary and multinomial deep autoencoders are denoted as bDA and $\mathrm{rsDA} .\left|\mathrm{Dim}_{i n}\right|$ is the dimensionality at the input layer.

$$
\begin{aligned}
\frac{\partial J(\theta)}{\partial \theta} & =\frac{\partial J(\theta)}{\partial h} \quad \frac{\partial h}{\partial z} \quad \frac{\partial z}{\partial \theta} \\
& =\frac{\partial}{\partial h} \frac{1}{2}(y-h)^{2} \quad \frac{\partial h}{\partial z} \quad \frac{\partial z}{\partial \theta} \\
& =-(y-h) \quad \frac{\partial}{\partial z} \operatorname{sigm}(\mathbf{z}) \quad \frac{\partial z}{\partial \theta} \\
& =-(y-h) \quad h(1-h) \quad \frac{\partial}{\partial \theta} \mathbf{z} \\
& =-(y-h) \quad h(1-h) \quad \frac{\partial}{\partial \theta}(W * \mathbf{v}+\mathbf{b}) \\
& =-(y-h) \quad h(1-h) \quad * \mathbf{v} \quad \text { when } \theta=W \\
& =-(y-h) \quad h(1-h) \quad \text { when } \theta=\mathbf{b}
\end{aligned}
$$


These gradients for parameters $W$ and $\mathbf{b}$ can be backpropagated to the previous layers by using the chain rule. As shown in Eq. 3.14, the gradient at the $l^{\text {th }}$ layer can be computed in terms of the already known gradient of the $(l+1)^{t h}$ layer.

$$
\begin{aligned}
& \delta_{l}=\frac{\partial J(\theta)}{\partial \theta_{l}} \\
& =\frac{\partial J(\theta)}{\partial \mathbf{z}_{l+1}} \quad \frac{\partial \mathbf{z}_{l+1}}{\partial \mathbf{z}_{l}} \quad \frac{\partial \mathbf{z}_{l}}{\partial \theta_{l}} \\
& =\delta_{l+1} \quad \frac{\partial \mathbf{z}_{l+1}}{\partial \mathbf{h}_{l}} \quad \frac{\partial \mathbf{h}_{l}}{\partial \mathbf{z}_{l}} \quad \frac{\partial \mathbf{z}_{l}}{\partial \theta_{l}} \\
& =\delta_{l+1} \quad \frac{\partial}{\partial \mathbf{a}_{l}}\left(W_{l+1} * h_{l}+b_{l+1}\right) \quad \frac{\partial \mathbf{h}_{l}}{\partial \mathbf{z}_{l}} \quad \frac{\partial \mathbf{z}_{l}}{\partial \theta_{l}} \\
& =\delta_{l+1} \quad * \quad W_{l+1} \quad \mathbf{h}(1-\mathbf{h}) \quad \frac{\partial \mathbf{z}_{l}}{\partial \theta_{l}}
\end{aligned}
$$

\subsection{Optimisation}

Neural network parameters can be updated using an optimisation method called gradient descent. Gradient methods are first-order optimisation algorithms that converge towards a local optimum of an objective function by taking consecutive steps that are proportional to the gradient. If the algorithm moves towards local minima using steps that are proportional to the negative gradient, it is called gradient descent; and if it moves towards local maxima using steps that are proportional to positive gradient, it is called gradient ascent. Under gradient descent, parameters of a model at iteration $t$ are updated as shown in Eq. 3.15

$$
\theta^{(t+1)}=\theta^{(t)}-\alpha \delta^{(t)}
$$

where $\alpha$ is learning rate and $\delta$ denotes gradient.

Usually the gradients are accumulated over all the datapoints $(m)$ of the training set and the weights are updated as shown in Eq. 3.16 . 


$$
\theta_{w}^{(t+1)}=\theta_{w}^{(t)}-\alpha \sum_{i=1}^{m}\left(-\left(y_{i}-a_{i}\right) \cdot a_{i} \cdot\left(1-a_{i}\right) * \mathbf{v}_{i}\right)
$$

In practice, sometimes it is not possible to compute the sum of the gradient over all the datapoints in one pass before update due to computational issues, such as gradient might overflow, hence the updates are made at each datapoint. It is a common practice, when using gradient descent, that the dataset is randomly shuffled to avoid possible bias towards specific regions of the objective function. Then the updates are made as shown in Algorithm 1. This process is called stochastic gradient descent.

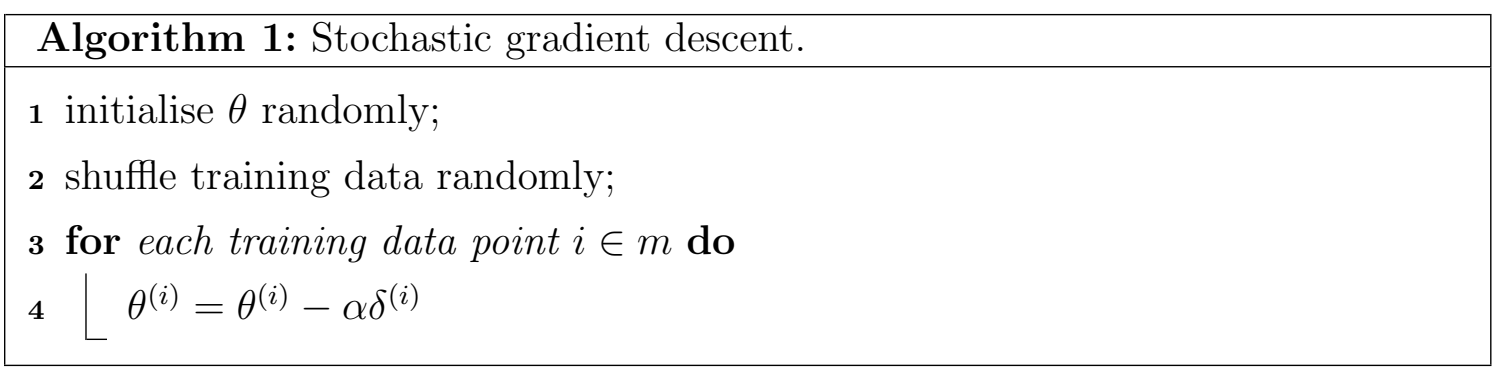

It is often shown that advanced optimisation methods such as conjugate gradient (Hestenes and Stiefel, 1952) or L-BFGS (Nocedal, 1980) are faster and are able to exhibit better convergence. Another common practice is to update the model parameters with gradients accumulated over a small batch of datapoints, instead of updating the model parameters after each datapoint or all datapoins. Such batches are called mini-batches. This kind of updates are also computationally efficient because they can better use multiple cores of CPUs or GPUs. 

$\Gamma_{\text {Chapter }}$

\section{Cross-view models}

This chapter aims at describing the main contributions of this thesis . Retrieval across different continuous space representations is central to the thesis contributions as described in Chapter 2. In recent years there is a surge and rapid growth in the neural network community around deep neural networks and deep learning. Data availability has grown large. This, along with a new break-through in computing research and especially in graphical processing units (GPUs), has allowed for often computationally expensive tasks such as training deep neural networks to become feasible from the experimental point of view. Most of the current research in deep learning is focused on single-embedding applications in different languages. This thesis focuses on the use of deep neural models for cross-embedding applications; more specifically two different cases are considered: cross-language and cross-script. In this chapter, we describe two novel techniques to capture similarities across different embeddings to aid cross-embedding retrieval: (i) cross-view autoencoder for mixed-script IR, and (ii) external data composition neural networks (XCNN) for cross-language IR. The cross-view autoencoder uses the RBM pretraining to initialise the network and 
then, the network is tuned using the autoencoder backpropagation. This architecture has shown promise in uncovering the meaningful representations (Hinton and Salakhutdinov, 2006). Hence, we take that architecture to cross-view setting, especially at character-level, to model mixed-script terms. On the other hand, there has been significant progress in terms of learning word-embeddings and representing text through composition (Mikolov et al., 2013; Hermann and Blunsom, 2014). In $\mathrm{XCNN}$, we learn bilingual word-embeddings through composition neural networks addressing some of the limitations of the present models.

\subsection{Cross-view autoencoder (CAE) for mixed-script IR}

As discussed in Chapters 2 and 5 , the primary challenge in mixed-script retrieval is to model and match terms across both scripts and spelling variations, which are especially common in the non-native scripts. We shall refer to the variants of the same word in the native and other scripts as term equivalent. The term matching problem can be addressed by using existing approaches such as approximate string matching (Hall and Dowling, 1980, Zobel and Dart, 1996) and transliteration mining (Udupa and Khapra, 2010a; Kumaran et al., 2010; Kumar and Udupa, 2011). The former is especially useful to handle spelling variations in a single script, while the latter can help in matching terms across the scripts. However, these methods cannot be directly used for term matching over a single and across multiple scripts at the same time.

In this section, we propose a framework for jointly modelling terms across scripts. We achieve this by learning a low-dimensional representation of terms in a common abstract space where term equivalents are close to each other. The concept of common abstract space for term equivalents is based on the fundamental observation that words are transliterated into a non-native script in such a way that its sound or pronunciation is preserved. Thus, if we can represent the pronunciation of words 
in an abstract space, it could faithfully embed terms written in any script in such a way that term equivalents are close to each other as far as they have similar pronunciations 1

\subsubsection{Formulation}

In order to build the intended models, we treat the phonemes as character-level "topics" within the terms. There is a good number of examples of word-level topic models using undirected graphical models like restricted Boltzmann machines (Gehler et al. 2006; Salakhutdinov and Hinton, 2009a b). These models are usually based on the assumption that each document is represented as a mixture of topics, where each topic defines a probability distribution over words. Similarly, in our proposed model, we consider the terms to be represented as a mixture of "topics", where each topic defines a probability distribution over character n-grams.

Phonemes can be captured by character n-grams. Consider the feature set $\mathcal{F}=$ $\left\{f_{1}, \ldots, f_{K}\right\}$ containing character grams of scripts $s_{i}$ for all $i \in\{1, ., r\}$ and $|\mathcal{F}|=K$. Let $t_{1}=\bigcup_{i=1 \ldots r} w_{1, i}$ be a datum from training data $T$ of language $l_{1}$, where $w_{1, i}$ represents a word $w$ written in language $l_{1}$ and script $s_{i}$, and $r$ is the number of scripts being modelled jointly. The datum can be represented as $K$-dimensional feature vector $\mathbf{x}$ where $x_{k}$ is the count of $k^{\text {th }}$ feature $f_{k} \in \mathcal{F}$ in datum $t_{1}$.

We assume that count of character grams within terms follow a Dirichlet-multinomial distribution. Consider $N$ independent draws from a categorical distribution with $K$ categories. In the present context, $N=\sum_{i}^{K} x_{i}$ and $\left\{f_{1}, \ldots, f_{K}\right\}$ are $K$ categories, where the number of times a particular feature $f_{k}$ occurs in the datum $t_{1}$ is denoted as $x_{k}$. Then $\mathbf{x}=\left(x_{1}, \ldots, x_{K}\right)$ follows a multinomial distribution with parameters $N$ and $\mathbf{p}$, where $\mathbf{p}=\left(p_{1}, \ldots, p_{K}\right)$ and $p_{k}$ is the probability that $k^{\text {th }}$ feature takes

\footnotetext{
${ }^{1}$ Not all scripts are used to represent the words according to their basic sounds or phonemes. For example, the Chinese script is a notable exception. However, most of the scripts (including Roman, Cyrillic, Arabic, Indic and other South Asian scripts) are more or less based on a phonemic orthography where words are broken down into syllables or phonemes and represented using letters. Hence, our method is applicable to these scripts.
} 
value $x_{k}$. The parameter $\mathbf{p}$ in our case is not directly available, hence we give it a conjugate prior distribution. Therefore, $\mathbf{p}$ is drawn from a Dirichlet distribution with parameter vector $\boldsymbol{\alpha}=\left(\alpha_{1}, \ldots, \alpha_{K}\right)$. The hyperprior vector $\boldsymbol{\alpha}$ can be seen as pseudocounts and $\alpha_{k}=x_{k} /\left(\sum_{i=1}^{K} x_{i}\right)$ in a reference collection. Such formulation can be expressed as follows:

$$
\begin{aligned}
\boldsymbol{\alpha} & =\left(\alpha_{1}, \ldots, \alpha_{k}\right)=\text { hyperprior } \\
\mathbf{p} \mid \boldsymbol{\alpha}=\left(p_{1}, \ldots, p_{K}\right) & \sim \operatorname{Dir}(K, \boldsymbol{\alpha}) \\
\mathbf{x} \mid \mathbf{p}=\left(x_{1}, \ldots, x_{K}\right) & \sim \operatorname{Mult}(K, \mathbf{p})
\end{aligned}
$$

The proposed model, CAE, is based on the non-linear dimensionality reduction method that uses a deep autoencoder (Hinton and Salakhutdinov, 2006). As already described in Sec. 3.4, in a deep autoencoder architecture, RBMs are stacked on top of each other. The bottom-most RBM of our model, which models the input terms, is a character-level variant of the replicated softmax model presented in (Salakhutdinov and Hinton, 2009b). Despite character n-grams follow a Dirichlet-multinomial distribution, we can model them by means of RSM because during the inference process, which uses Gibbs sampling, Dirichlet prior distributions are marginalised out. Let $\mathbf{v} \in\{0,1, \cdots, N\}^{K}$ represent visible multinomial units and let $\mathbf{h} \in\{0,1\}^{m}$ be stochastic binary hidden latent units. Let $\mathbf{v}$ be a $K$-dimensional input vector such as feature vector of features $x_{i}, \mathbf{h}$ an $m$-dimensional latent feature vector and $N=\sum_{i=1}^{K} x_{i}$. The energy of the state $\{\mathbf{v}, \mathbf{h}\}$ is defined as:

$$
E(\mathbf{v}, \mathbf{h})=-\sum_{i=1}^{K} v^{i} a^{i}-N \sum_{j=1}^{m} b_{j} h_{j}-\sum_{i, j} W_{j}^{i} h_{j} v^{i}
$$

where $v^{i}$ is the corresponding count for feature $x_{i}, W_{j}^{i}$ is the weight matrix entry between the $i^{\text {th }}$ visible node and the $j^{\text {th }}$ hidden node, while $a^{i}$ and $b_{j}$ are the bias terms of the visible and hidden layers respectively. The resulting conditional distributions 
are given by the softmax and logistic functions, are as below:

$$
\begin{gathered}
p\left(v^{i}=x_{i} \mid \mathbf{h}\right)=\frac{\exp \left(a^{i}+\sum_{j} h_{j} W_{j}^{i}\right)}{\sum_{i=1}^{K} \exp \left(a_{i}+\sum_{j} h_{j} W_{j}^{i}\right)} \\
p\left(h_{j}=1 \mid \mathbf{v}\right)=\sigma\left(b_{j}+N \sum_{i=1}^{K} v_{i} W_{j}^{i}\right)
\end{gathered}
$$

As argued in Salakhutdinov and Hinton (2009a), a single layer of binary features may not be the best way to capture complex structures in the data, then more layers are added to create a deep autoencoder (Hinton and Salakhutdinov, 2006). The further binary RBM's are stacked on top of each other in such a way that the output of the bottom RBM is the input to the above RBM. The conditional distributions of these binary RBMs are given by logistic functions as follows:

$$
\begin{aligned}
& p\left(v^{i}=1 \mid \mathbf{h}\right)=\sigma\left(a^{i}+\sum_{j} h_{j} W_{j}^{i}\right) \\
& p\left(h_{j}=1 \mid \mathbf{v}\right)=\sigma\left(b_{j}+\sum_{i} v^{i} W_{j}^{i}\right)
\end{aligned}
$$

\subsubsection{Closed feature set - finite $K$}

Topic models for documents are usually trained over a subset of vocabulary (top-n terms) and hence, they have to deal with the non-trivial problem of marginalising over unobserved terms. On the contrary, our proposed term level topic model is prune to this problem because the set of phonemes (more specifically, character ngrams) for a given language is finite and typically small. Hence, enough evidence for all the phonemes is found even in a small to moderate size training dataset, which increases the suitability of our approach to the problem.

For example, without loss of generality, consider the total number of scripts in 


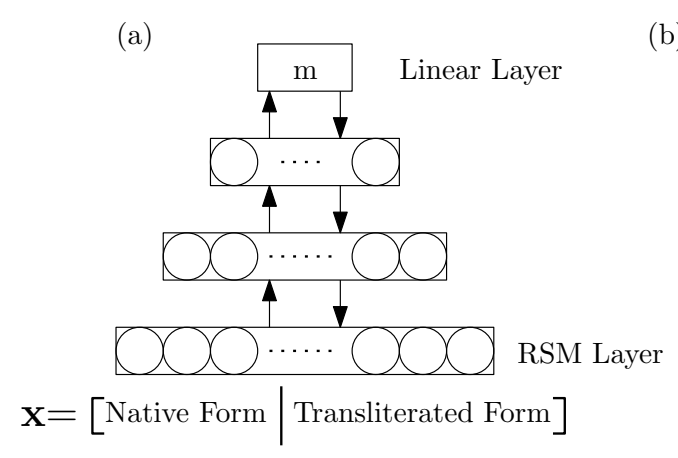

(b)

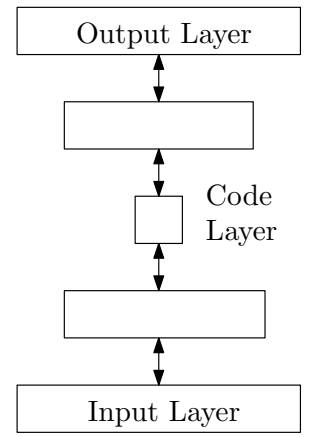

(c)

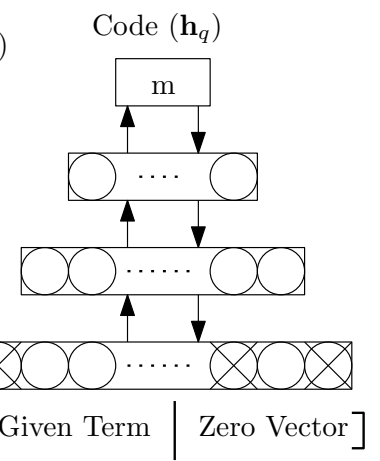

Figure 4.1: The architecture of the autoencoder (K-500-250-m) during (a) pre-training and (b) fine-tuning. After training, the abstract level representation of the given terms can be obtained as shown in (c).

datum being modelled $r=2$ for language Hindi where $s_{1}$ be the Devanagari script with 50 letters and $s_{2}$ be the Roman script (as used in English orthography) with 26 letters. Then, the size of the feature set $\mathcal{F}$, considering uni-gram and bi-gram character features, is upper bounded by $K=3252=\left(26+26^{2}+50+50^{2}\right)$.

\subsubsection{Training}

The architecture of the proposed mixed-script autoencoder is shown in Fig. 4.1 (a). The visible layer of the bottom-most RBM is a character level replicated softmax layer as described in Sec.4.1.1. The character uni-grams and bi-grams of the training datum $(r=2)$ constitute the feature space $\mathcal{F}$. The hidden layer of the top-most RBM is linear and represents the low-dimensional embedding of terms in the abstract space. As already described in autoencoder is trained in two phases: $(i)$ greedy layer-wise pre-training and; (ii) fine-tuning through backpropagation.

(i) Layer-wise pre-training Multilayer neural network training is known to suffer from the vanishing gradient problem, the gradient at the bottom layer becomes small because of many small-number multiplications. Greedy layer-wise pre-training brought a break-through in training deep neural network architectures, where each 


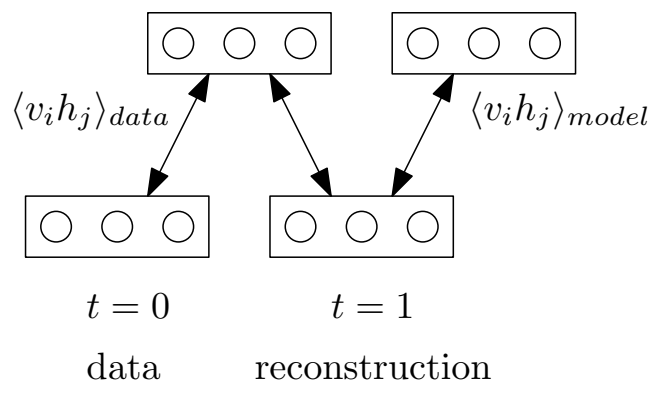

Figure 4.2: Contrastive divergence technique to pre-train RBM

layer is trained individually. Each new layer guarantees to increase the lower bound of the log-likelihood of the data, which in turn improves the model (Hinton and Salakhutdinov, 2006).

During pre-training, each RBM is trained using contrastive divergence (CD) learning with $n$ alternating Gibbs sampling (Hinton, 2002). Under this learning, the update rule becomes simple as shown in Eq. 4.6, where $\left\langle v_{i} h_{j}\right\rangle_{\text {data }}$ represents the expectations under the original data distribution and $\left\langle v_{i} h_{j}\right\rangle_{\text {model }}$ represents the expectations under the model distribution. In practice, a single alternating Gibbs sampling gives good results, which can be denoted as $\mathrm{CD}_{1}$ learning. The greedy pre-training is shown in Fig. 4.2. It is also noted that pre-training helps to initialise the network parameters in a region with high probability of finding global optimum (Erhan et al., 2010).

$$
\Delta w_{i j}=\epsilon\left(\left\langle v_{i} h_{j}\right\rangle_{\text {data }}-\left\langle v_{i} h_{j}\right\rangle_{\text {model }}\right)
$$

(ii) Fine tuning Once the network is pre-trained, the autoencoder is unrolled as shown in Fig. 4.1 (b) and the cross-entropy error (Eq. 4.7) between the input and its reconstruction (output) is minimised by using backpropagation to adjust the weights of the entire network.

$$
J(\theta)=-\mathbf{x} \log (\hat{\mathbf{x}})-(1-\mathbf{x}) * \log (1-\hat{\mathbf{x}})
$$


As shown in Fig. 4.1 (a), the autoencoder is jointly trained with a native form and its transliterated form. In this way, the model is able to learn character level "topic" distributions over the features of both scripts jointly.

\subsubsection{Finding equivalents}

Once the model is trained, equivalents discovery involves two steps: $(i)$ preparing the index of mining lexicon in the abstract space (offline) and; (ii) finding equivalents for a given query term (online). The lexicon of the reference collection (ideally crossscript), which is used to find term equivalents is referred to as the mining lexicon. The former step is a one-time offline process in which the $m$-dimensional abstract representation for each term in mining lexicon (of size $n$ ) is obtained as shown in Fig. 4.1 (c) $\left(\mathbf{x}_{1 \times K} \rightarrow \mathbf{h}_{1 \times m}\right)$. These representations are stored in an index against each term. This index can be seen as an $n \times m$ matrix $\mathbf{H}$ where $\mathbf{h} \in \mathbf{H}$. The

latter step involves projecting the query term into the abstract space $\left(\mathbf{x}_{q} \rightarrow \mathbf{h}_{q}\right)$ and calculating the similarity with all the terms in the index. The similarity calculation can be seen as a matrix multiplication operation $\mathbf{H} \mathbf{h}_{q}^{T}$, in which the cosine similarity function is considered. All the terms with $\operatorname{sim}\left(\mathbf{h}, \mathbf{h}_{q}\right)>\theta, \mathbf{h} \in \mathbf{H}$ are considered as term equivalents of the query word $w_{q}$, where $\theta$ is similarity threshold.

\subsection{External data composition neural networks}

The cross-view autoencoder described in the previous section can also be used to model cross-language documents, although it does not perform as strongly as it does for modelling mixed-script equivalents. There are other limitations: $(i)$ it does not provide an explicilt way to incorporate external relevance signals such as clickthrough data, which is very helpful for information retrieval tasks; and (ii) it learns crosslanguage representations by optimising identity function which is loosely related to the evaluation metric of retrieval. In this section we introduce external data composition neural networks, which is a novel method to learn term associations 
across languages in a distributed manner to aid cross-language information retrieval. In contrast to most of the existing models, which rely only on comparable and/or parallel data, our model takes into account external relevance signals such as pseudorelevance or clickthrough data. This external data is used to initialise monolingual embeddings and then, with the use of a small amount of parallel data, the parameters for the different languages are jointly adjusted. The proposed framework also allows for the use of clickthrough data, if available, instead of pseudo-relevance data. Our model, differently from other models, optimises an objective function that is directly related to an information retrieval evaluation metric, such as cosine similarity. These two properties prove crucial for our model to outperform existing techniques in crosslanguage IR setting.

Most prior work on learning low-dimensional semantic representations across languages relies completely on parallel data for training the models (Platt et al., 2010; Yih et al., 2011; Gupta et al., 2014). Our proposed framework removes this requirement by exploiting also monolingual data for model training purposes, and as such it can be more easily applied to low-resource languages.
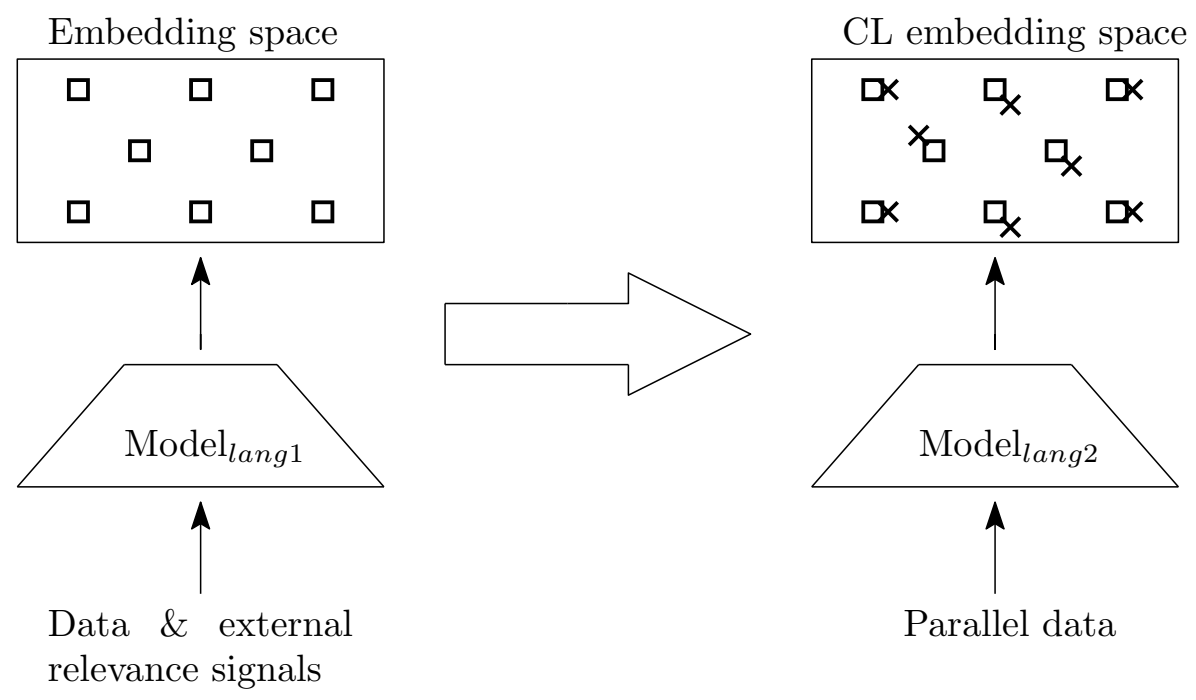

Figure 4.3: System overview of training of XCNN model.

In summary, we attempt to incorporate external relevance signals such as pseudo- 
relevance or clickthrough data into the learning framework. Such data might not be available across languages and is mostly restricted to the monolingual setting, as most of the present search engines do not perform cross-language retrieval explicitly. The main idea behind our proposal is that, monolingual models can be intialised from such largely available external data and then, with the help of a smaller amount of parallel data, the cross-language model can be trained. This property helps to gain more confidence for under-represented terms in the parallel data, i.e. terms with very low frequency. The overview of the XCNN model can be depicted as shown in Fig. 4.3 .

The low dimensional embedding space created through monolingual data and external relevance signal is then extended cross-lingually as shown in Fig. 4.4. In Fig. 4.4, text in language-1 ( lang $\left._{1}\right)$ is represented by symbol $\square$ while the corresponding parallel text in language-2 $\left(l_{a n g_{2}}\right)$ is represented by symbol $\times$ and the arrows show the parallel correspondance. Before training, the $\operatorname{lang}_{2}$ text is represented in the embedding space when the corresponding model is randomly initialised. The lang $_{2}$ model parameters are updated to obtain a cross-view embeddings space as shown in the right hand side of Fig. 4.4.

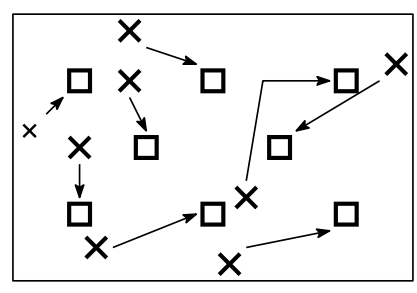

Before training

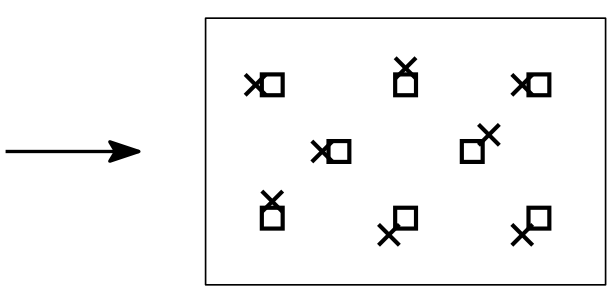

After training

Figure 4.4: Embedding space before and after cross-lingual extension training.

\subsubsection{Monolingual pre-initialisation}

The monolingual pre-initialisation can be performed by means of any monolingual latent semantic modelling approach. In our proposed method, we consider a model 
similar to the deep semantic structured model (Huang et al., 2013) with two modifications: $(i)$ we do not use word-hashing as we will extend this model to the crosslanguage framework and we are more interested in word associations, and (ii) we use a composition function to feed the text into the model rather than a standard bag-of-word vector representation.

Consider a function $f: x \rightarrow y \in \mathbb{R}^{d}$, which maps a document vector $x$ into a distributed semantic representation $y$. We use a simple additive vector composition function on top of the deep neural network output. The architecture of the composi-

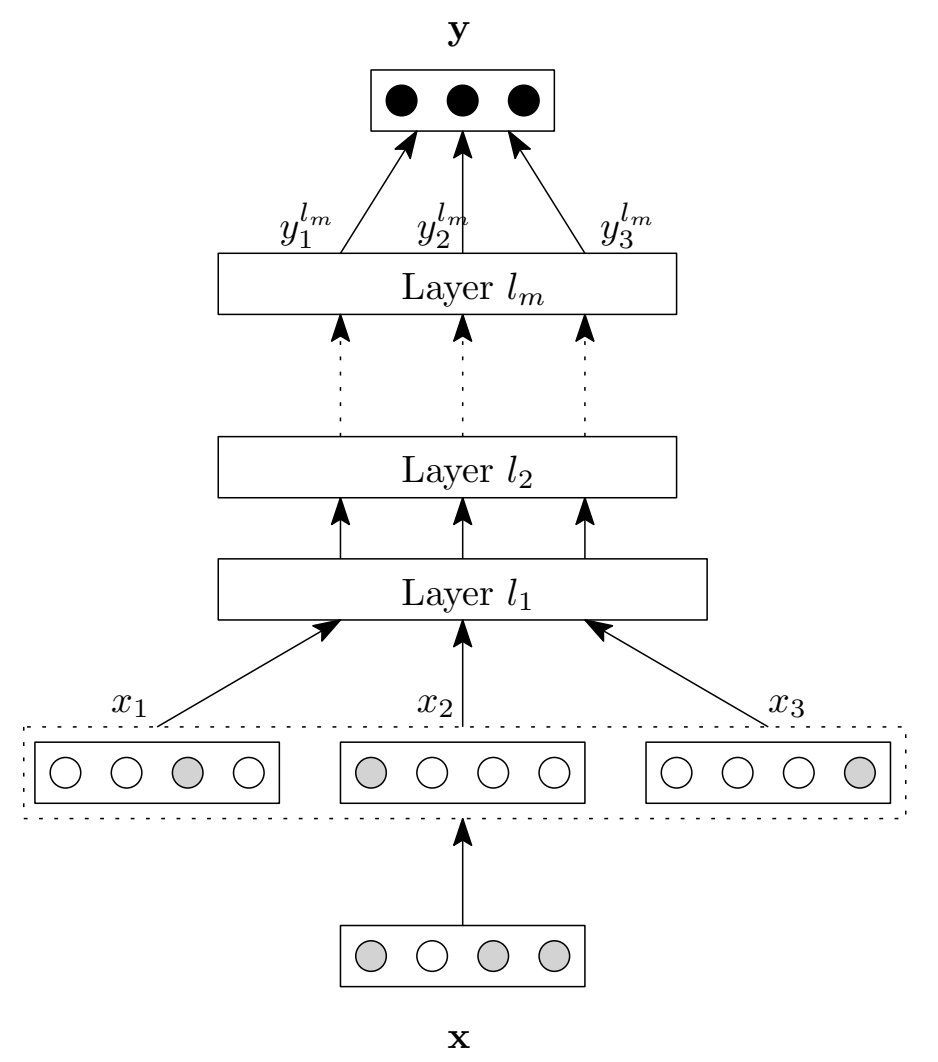

Figure 4.5: Composition Model.

tion model with $m$ layers is shown in Fig. 4.5. The input layer accepts the document vector $x$ and the output layer $\left(l_{m}\right)$ provides the semantic representation for the document vectors. In our approach, one-hot representation of each term $x_{i}$ is obtained 
from bag-of-words document vector $x$ as shown in Fig. 4.5. The hidden layer activities and the semantic representation $y$ are obtained by means of Eq. 4.8. As it can be noticed in Eq. 4.8, an additive composition is performed over the representation of terms in the output layer $\left(l_{m}\right)$.

$$
\begin{aligned}
y_{i}^{l_{1}} & =g\left(W_{1} * x_{i}+b_{1}\right) \\
y_{i}^{l_{j}} & =g\left(W_{j} * y_{i}^{l_{j-1}}+b_{j}\right), j=2, \ldots, m \\
y & =\sum_{i=1}^{n} y_{i}^{l_{m}}
\end{aligned}
$$

where $W_{j}$ and $b_{j}$ are the $j^{\text {th }}$ layer weights and biases respectively, $n$ is the total number of terms in the document and $g(z)$ is a non-linear activation function. In our approach we use the hyperbolic tangent for non-linearity:

$$
g(z)=\tanh (z)=\frac{1-e^{-2 z}}{1+e^{-2 z}}
$$

This composition framework is slightly different from the standard bag-of-words representation of documents used with feed-forward neural network because the terms are added after applying the non-linearity.

The architecture of the proposed monolingual pre-initialisation model is depicted in Fig. 4.6. This model is trained to maximise the following objective function:

$$
J(\theta)=\cos \left(y_{Q}, y_{D^{+}}\right)-\cos \left(y_{Q}, y_{D^{-}}\right)
$$

where $\cos \left(y_{Q}, y_{D}\right)$ denotes the cosine similarity between the semantic representations of query $(Q)$ and document $(D)$ as shown below:

$$
\operatorname{sim}\left(y_{Q}, y_{D}\right)=\cos \left(y_{Q}, y_{D}\right)=\frac{{\overrightarrow{y_{Q}}}^{T} \overrightarrow{y_{D}}}{\left\|\overrightarrow{y_{Q}}\right\|\left\|\overrightarrow{y_{D}}\right\|}
$$

Maximising the proposed objective function reinforces the cosine similarity between 


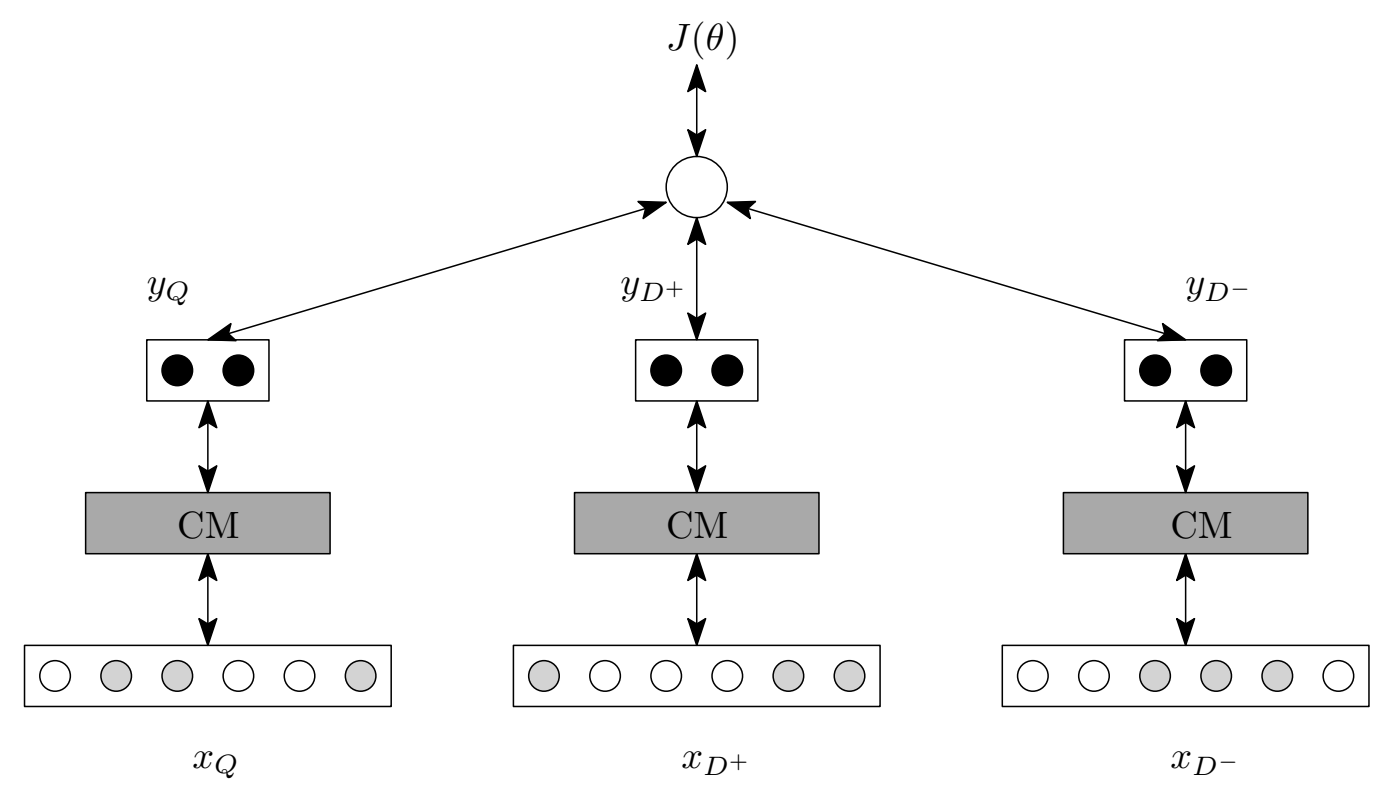

Figure 4.6: Relevance backpropagation model for monolingual pre-initialisation of the latent space using monolingual relevance data.

relevant document (positive sample, $D^{+}$) and query $(Q)$ to be high and the similarity between irrelevant document (negative sample, $D^{-}$) and the query $(Q)$ to be low. The noise-contrastive component $\left(\cos \left(y_{Q}, y_{D^{-}}\right)\right)$prevents the model from over-fitting and helps improving generalisation. During the training, the model parameters are updated using a gradient method, which was already described in Section 3.6. For brevity and consistency, the details of the gradient derivation for the objective function in Eq. 4.10 are given in Appendix A.1.

\subsubsection{Cross-language extension}

The main idea of the proposed framework is to implement a cross-language representation in a semi-supervised manner with a limited set of parallel data. To achieve this, we first project one side of the parallel data by using its corresponding monolingual model. Then, we tune the opposite monolingual model with the use of the other side of the parallel data. We call the tuned model the cross-language extension 
model.

Consider a 3 -tuple $\left(y_{l_{1}}, y_{l_{2}}^{+}, y_{l_{2}}^{-}\right)$, where $l_{1}$ is the language for which we are training the cross-language extension model, $y_{l_{1}}$ denotes the distributed representation of term vector $x$ in $l_{1}$. On the other hand, $y_{l_{2}}^{+}$denotes the distributed representation of the parallel counterpart of $x$ in $l_{2}$ and $y_{l_{2}}^{-}$is a noise component in $l_{2}$. The overall architecture of the model is depicted in Fig. 4.7 and the corresponding objective function is:

$$
J_{c l}(\theta)=\cos \left(y_{l_{1}}, y_{l_{2}}^{+}\right)-\cos \left(y_{l_{1}}, y_{l_{2}}^{-}\right)
$$

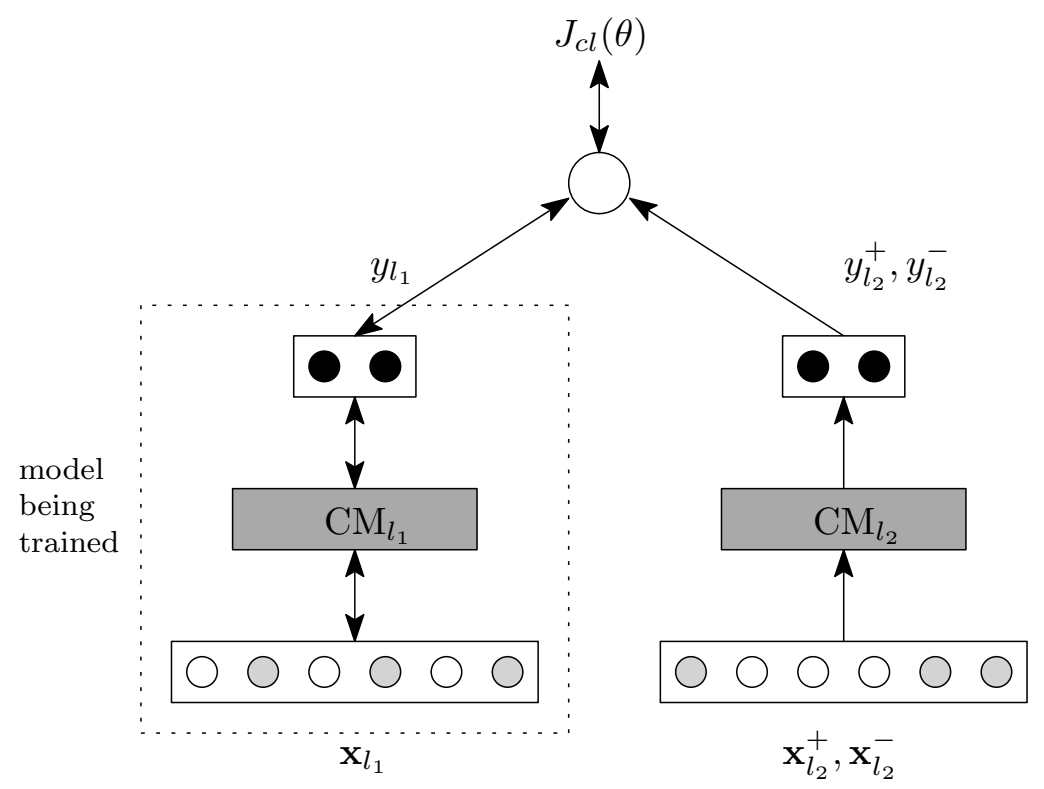

Figure 4.7: Cross-lingual extension model.

The composition models $\mathrm{CM}_{l_{2}}$ are obtained through monolingual pre-initialisation. In the cross-language extension phase, only the model parameters of $\mathrm{CM}_{l_{1}}$ are updated during the training. The details of the gradient derivation for the objective function presented in Eq. 4.12 are given in Appendix A.2. 



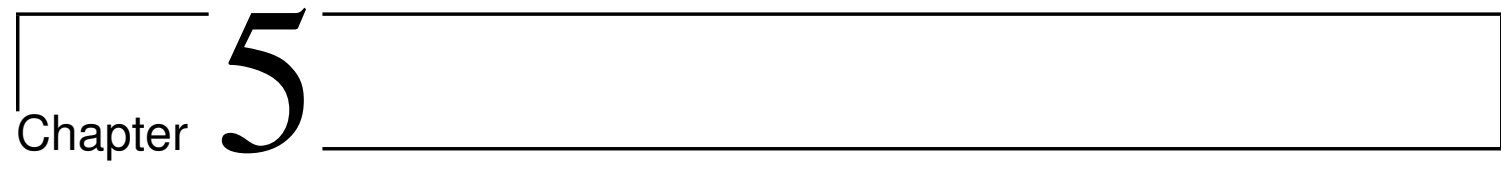

\section{Mixed-script information retrieval}

For many languages that use non-Roman indigenous scripts (e.g., Arabic, Greek and Indic languages), one can often find a large amount of transliterated user generated content on the web in the Roman script. Such content creates a monolingual or multi-lingual space with more than one script which we refer to as the mixed-script space. IR in this mixed-script space is challenging because queries written in either the native or the Roman script need to be matched to the documents written in both scripts. Moreover, transliterated content features extensive spelling variations. In this chapter, the concept of mixed-script IR is formally introduced (Section 5.1). Through analysis of the query logs of Bing search engine, the prevalence and importance of this problem is estimated (Section 5.2). Finally, the experiments and results on a standard dataset are reported with the proposed model in this thesis and compared to variety of strong baselines in Sec. 5.3 . 


\subsection{MSIR: Definition \& challenges}

In this section, the notion of mixed-script IR is formally defined along the lines of cross-lingual IR (Gupta et al., 2014; Gupta, 2014). A set of research challenges in the context of MSIR are also presented.

\subsubsection{Languages, scripts and transliteration}

Let $\mathcal{L}$ be a set of (natural) languages $\left\{l_{1}, l_{2}, \ldots, l_{n}\right\}$. Assuming that every language is generally written using a particular script, which is referred to as the native script of the language. Let $s_{i}$ be the native script for language $l_{i}$. Thus, the set of scripts $\mathcal{S}=\left\{s_{1}, s_{2}, \ldots, s_{n}\right\}$ has a one-to-one mapping to $\mathcal{L}$.

Any natural language word $w$ has two attributes: the language it belongs to and the script it is written in. The notation $w \in\left\langle l_{i}, s_{j}\right\rangle$ implies that $w$ is in language $l_{i}$, written using the script $s_{j}$. When $i=j$, word $w$ is considered to be in native script. Else, in transliterated form, where transliteration can be defined as the process of loosely or informally representing the sound of a word of one language, $l_{i}$ using a non-native script $s_{j}$.

Note that a particular language might be traditionally written in more than one script. For instance, Kurdish is written using the Roman, Cyrillic and Arabic scripts. However, such cases are rare. On the other hand, it is very common to use a script for writing several languages. For instance, the Roman script (with slight variations or additions of diacritics) is used to write English, French, German, Italian, Turkish and many other languages around the world. Similarly, the Devanagari script is used for writing Hindi, Sanskrit, Nepali and Marathi languages. Our definition does not preclude such a possibility, but we would like to emphasise that it is useful to treat the same script differently when used for writing different languages because the same sequence of letters might have different pronunciations in different languages. Consequently, transliterating a word of $l_{i}$ (say Hindi) into the scripts $s_{j}$ (say Roman script as used in English orthography) and $s_{k}$ (say Roman script as used in French 
orthography) could yield very different results, even though the two scripts use almost an identical alphabet.

\subsubsection{Mixed-script IR}

Given a query $q$ and a document pool $\mathcal{D}$, the task of an IR engine is to rank the documents in $\mathcal{D}$ such that the ones relevant to $q$ appear at the top of the ranked list. Depending on the language in which $q$ and $\mathcal{D}$ are presented, one can define two basic kinds of IR settings. Without loss of generality, let us assume that $q \in\left\langle l_{1}, s_{1}\right\rangle$. In monolingual IR, $\mathcal{D}=\left\{d_{1}, d_{2}, \ldots, d_{N}\right\}$ consists of only those documents that are in the same language and script as the query, i.e., for all $k, d_{k} \in\left\langle l_{1}, s_{1}\right\rangle$. This simple scenario is modified in the context of CLIR, where

$$
\mathcal{D}=\bigcup_{i=1 \ldots n} \mathcal{D}_{i}
$$

where $\mathcal{D}_{i}=\left\{d_{i}^{1}, d_{i}^{2}, \ldots, d_{i}^{N}\right\}$ are documents in language $l_{i}$, i.e., for all $k, d_{i}^{k} \in\left\langle l_{i}\right.$, $\left.s_{i}\right\rangle$. Note that all the documents in a typical CLIR setup are assumed to be written in the corresponding native scripts.

Based on this fundamental idea of CLIR, a corresponding mixed-script IR setup can be defined as follows. Let $q \in\left\langle l_{1}, s_{j}\right\rangle$ be a query, where $j$ may or may not be equal to 1 . The document pool,

$$
\mathcal{D}=\bigcup_{k=1 \ldots n} \mathcal{D}_{1}^{k}
$$

where $\mathcal{D}_{1}^{k}=\left\{d_{1}^{k, 1}, d_{1}^{k, 2}, \ldots, d_{1}^{k, N}\right\}$ are documents in language $l_{1}$ written in script $s_{k}$, i.e., for all $m, d_{1}^{k, m} \in\left\langle l_{1}, s_{k}\right\rangle$. In other words, in the MSIR setup, the query and the documents are all in the same language, say $l_{1}$, but they are written in more than one different scripts. The task of the IR engine is to search across the scripts.

In the literature, sometimes CLIR is distinguished from multilingual IR in the sense that the former refers to a case where $n=2$, whereas the latter is a gener- 
alization to any $n>2$. Likewise, for monolingual IR, $n$ can be assumed to be 1 . One could make a similar distinction between mono-script, cross-Script and mixedscript IR scenarios, where the query and the documents are in one language, but in 1, 2 or more than 2 scripts respectively. Nevertheless, we will refer to both latter cases as MSIR. All the experiments involve a single language, namely Hindi, and two scripts - Devanagari and the Roman script (English orthography) - but the proposed approach can be easily extended to a larger set of scripts.

One can also further generalise the setup to mixed-script multilingual IR, where $q$ as well as $\mathcal{D}$ can be in one of several languages and written in one of several scripts. This is also a useful and practical setup, though we will not discuss it any further in this work.

It should also be noted that, like in CLIR, in the MSIR setting it is possible that for $q \in\left\{l_{i}, s_{j}\right\}$, the information might be available only in a $d_{i}^{j^{\prime}, k}$ where $i \neq j^{\prime}$. In such cases, often the users issuing the query might be able to read and write both $s_{j}$ and $s_{j^{\prime}}$ and hence $d_{i}^{j^{\prime}, k}$ would have solved users information need. However, without MSIR this would not be possible to achieve.

\subsubsection{Mixed and transliterated queries \& documents}

The definition of the MSIR setup assumes that the entire query and each document are in a single language and single script. However, in practice, one can find queries or documents that contain text fragments written in more than one language or script or both. Furthermore, depending on whether the parts of a query or document are written in a language using the native or a non-native script, one can have native or transliterated queries and documents.

A practical way to address the issue of mixed-script within documents could be to split them into several sub-documents such that each of the sub-documents are in a single language and single script as discussed in Choudhury et al. (2012), given the mixing is not at the sub-sentence level which falls under the different case of codemixing. Mixed queries, however, cannot be handled through simple splitting because 
matching parts of a query to the documents does not make sense in the context of IR. Therefore, the MSIR setup is extended to include mixed queries. Let a query $q$ be defined as a string of words $w_{1} w_{2} \ldots w_{m}$, where $w_{1} \in\left\langle l_{i_{1}}, s_{j_{1}}\right\rangle, w_{2} \in\left\langle l_{i_{2}}, s_{j_{2}}\right\rangle$ and so on can all belong to different languages, scripts or both.

\subsubsection{Challenges in MSIR}

The two primary challenges in MSIR are: (i) how to tackle the extensive spelling variations in the transliterated queries and documents during the term matching phase, and (ii) how to identify, process and represent a mixed query (and also, the mixed and transliterated documents). In CLIR, there are broadly two approaches: (i) to model the cross-lingual space, either documents and queries are translated to bring all words into the same monolingual space, after which monolingual IR techniques and matching algorithms can be directly applied; or (ii) the cross-lingual space is modelled jointly as an abstract topic or semantic space, and documents and queries in all languages are mapped to this common space. Likewise, in MSIR one can "transliterate" the text to bring everything into a common space and then apply standard matching techniques in the single-script space, or one can jointly model an abstract orthographic space for representing the words written in different scripts. In this thesis, we explore the latter, which we believe is a more robust and generic solution to the mixed-script space modelling problem, as it can simultaneously handle spelling variations in a single script and across multiple scripts.

Mixed query processing is another interesting research challenge, which includes language identification of the query words, which can be either in native or transliterated scripts, and labeling those with semantic or other tags (e.g., entities, attributes). This is challenging mainly because, depending on the context of the query, the same string, say "man", could represent the English word man, a transliterated Hindi word man meaning "mind", or another transliterated Hindi word maan meaning "reputation". In addition, the same word with similar meanings are also used in many other Indian languages and can have different connotations in other languages (e.g., 
in Bengali this could also mean "to get offended"). Hence, language identification seems to be an extremely challenging problem in the MSIR setting, especially when multiple languages are involved. In this work, we limit our experiments to only two languages, namely English and Hindi, and describe some initial results with language identification for transliterated and mixed queries.

Apart from these basic challenges, result presentation in MSIR is also an interesting problem because this requires the information on whether the user can read all the scripts, or prefer some scripts over others. There are no user studies related to MSIR, which is ripe with several such open problems.

\subsection{Transliterated queries in web search}

Although the current web search engines do not support MSIR, they still have to handle a large traffic of mixed and transliterated queries from linguistic regions that use non-Roman indigenous scripts. To better understand the distribution of transliterated queries across various topics and domains, an analysis of mixed and transliterated queries extracted from a large query log of Bing is presented. This could provide deeper understanding of the MSIR space and its users. This analysis relies on automatic identification and classification techniques for mixed queries developed specifically for this task.

\subsubsection{Methodology}

The analysis is conducted on 13.78 billion queries sampled from the logs of Bing on real user searches conducted in India. India provides an interesting socio-linguistic context for studying mixed queries because of the abundance of Roman transliterations and the multiplicity of languages and scripts. This dataset consists of 30 million unique queries with an average length of 4.32 words per query. Almost all the queries (99.998\%) are in Roman script (but not necessarily in English language). For ease of computation, we randomly sampled $1 \%$ (i.e., 300,000 ) of the unique queries and 
conducted the study on this smaller sample. The analysis is carried out in successive steps, which are explained below.

Step-1: Language identification In order to identify the mixed-script queries, a language identification classifier was trained. The classifier is based on a maximum entropy model, which uses character $n$-gram features $(n=1$ to 5$)$. Training was carried out with 5000 labelled words for each language. Hindi words were top transliterated words from Bollywood songs lyrics obtained through Gupta et al. (2012a) and English words from the Leipzig Corpus ${ }^{1}$. On 2500 unseen words, the accuracy of the classifier was measured to be $97 \%$. The language identification was carried out based on a similar word-level identification task in King and Abney (2013). With this classifier, a query $q$ is considered to be mixed-script or transliterated if it contains more than $40 \%$ words classified as Hindi.

Step-2: Query categories After analysing the transliterated queries identified in Step-1, six broad categories or topics were identified: Named Entities, Entertainment, Information Source, Culture, Recipe and Research. Each of these were further refined into a set of sub-categories; e.g., Named Entities can be of three types people, location and organisation. Besides, we also observed a few interesting subcategories, which we put together under a catch-all seventh category - Others. Table 5.1 lists all these categories and sub-categories along with example queries.

Step-3: Category assignment In order to automatically classify the queries into these categories, we resort to a simple minimally supervised approach. Through manual inspection of the transliterated queries, five representative and reasonably frequent examples for each sub-category were selected. All the queries from the dataset that have at least one word in common with at least one of the five representative queries were extracted. Then, the top 100 most frequent words in this set of queries were populated. The standard English stopwords were removed from these 100

\footnotetext{
${ }^{1}$ http://corpora.uni-leipzig.de/
} 


\begin{tabular}{|c|c|c|c|}
\hline Category & |Sub-categories & Cue words & Example query \\
\hline Named Entity & $\begin{array}{l}\text { People } \\
\text { Organisation } \\
\text { Location }\end{array}$ & $\begin{array}{l}\text { mr, ji, guru, dr, swami } \\
\text { ltd, university, bank } \\
\text { nagar, garh, chowk, hotel }\end{array}$ & $\begin{array}{l}\text { harmohinder singh } \\
\text { gogia } \\
\text { gandharva } \\
\text { mahavidyalaya ddu } \\
\text { marg } \\
\text { rajdhani train } \\
\text { timings chappra to } \\
\text { guwhati }\end{array}$ \\
\hline Entertainment & $\mid \begin{array}{l}\text { Movie } \\
\text { Song/Lyrics/Dialogues } \\
\text { Tv soaps/serials }\end{array}$ & s $\begin{array}{l}\text { movie, film, torrent, video } \\
\text { album, tune, lyrics, audio } \\
\text { song, lyrics, tv, serial }\end{array}$ & $\begin{array}{l}\text { himmatwaala remake } \\
\text { ik din ayega lyrics } \\
\text { colors madhubala } \\
\text { ishq ek junoon }\end{array}$ \\
\hline $\begin{array}{l}\text { Information } \\
\text { Source }\end{array}$ & $\begin{array}{l}\text { Books } \\
\text { magazines/news } \\
\text { websites }\end{array}$ & $\begin{array}{l}\text { book, pustak, kitab } \\
\text { patrika, times, } \\
\text { blog, com, net, http }\end{array}$ & $\begin{array}{l}\text { bade ghar } k i \text { beti } \\
\text { premchand } \\
\text { vasundhara eenadu } \\
\text { swayamvaram info }\end{array}$ \\
\hline Culture & $\begin{array}{l}\text { Religion } \\
\text { Art/Literature } \\
\text { Astrology } \\
\text { Attire }\end{array}$ & $\begin{array}{l}\text { festival, god, lord } \\
\text { yoga, natyam, raaga } \\
\text { rashi, horoscope, kundali } \\
\text { saree, sherwani, lehenga }\end{array}$ & $\begin{array}{l}\text { ahoi ashtami } 2011 \\
\text { bharatanaytam dance } \\
\text { kalakshetra } \\
\text { ashwini nakshatra } \\
\text { mesha raashi } \\
\text { silk bandhni } \\
\text { chaniya choli }\end{array}$ \\
\hline Recipe & Recipe/Dish/Food & curry, biryani, paneer & $\begin{array}{l}\text { matar panir by } \\
\text { tarala dalal }\end{array}$ \\
\hline Research & Economic/Agriculture & arthik, samaj & $\begin{array}{l}\text { vishwa arthik mandi } \\
\text { mein bharat }\end{array}$ \\
\hline Others & |- & meaning & vibhaa meaning \\
\hline
\end{tabular}

Table 5.1: Classification of transliterated Hindi queries. Transliterated words are italicised.

words. The remaining words constitute what we refer as the cue words for the particular subcategory. A total of 180 cue words were obtained for each sub-category, with very few overlaps. Some example cue words for each of the sub-categories are 
reported in Table 5.1 .

Let $c_{1}^{j}$ to $c_{m_{j}}^{j}$ be the cue words associated with the $j^{\text {th }}$ sub-category. For each of the transliterated queries $q=w_{1} w_{2} \ldots w_{n}$ that we want to categorise, we remove all the stopwords and cue words. For each of the remaining words in the query, say $w_{i}$, we count the number of queries, $f_{i, k}^{j}$, in the log where $w_{i}$ co-occurs with the cue-word $c_{k}^{j}$. Also, let $f_{i}$ be the number of queries in which $w_{i}$ occurs. We compute the score of $q$ with respect to a sub-category $j$ as:

$$
\operatorname{score}(q, j)=\sum_{i=1}^{k} \sum_{k=1}^{m_{j}} f_{i, k}^{j} / f_{i}
$$

where, $q$ is assigned to the sub-category $j^{*}$ for which this score is maximum.

\begin{tabular}{l|l|r|r}
\hline Category & Sub-categories & \% of Unique & \% of Total \\
\hline \multirow{3}{*}{ Named Entity } & People & $6 \%$ & $1.04 \%$ \\
& Organisation & $14 \%$ & $2.8 \%$ \\
& Location & $8 \%$ & $2.13 \%$ \\
\hline \multirow{3}{*}{ Entertainment } & Movie & $7 \%$ & $19.56 \%$ \\
& Song/Lyrics/Dialogues & $18 \%$ & $12.8 \%$ \\
& Tv soaps/serials & $2 \%$ & $0.62 \%$ \\
\hline \multirow{2}{*}{ Information } & Books & $0.005 \%$ & $0.02 \%$ \\
Source & Magazines/news & $3 \%$ & $14.52 \%$ \\
& Websites & $22 \%$ & $44.18 \%$ \\
\hline \multirow{3}{*}{ Culture } & Religion & $0.4 \%$ & $0.02 \%$ \\
& Art/Literature & $0.3 \%$ & $0.01 \%$ \\
& Astrology & $0.3 \%$ & $0.2 \%$ \\
& Attire & $0.3 \%$ & $0.04 \%$ \\
\hline Recipe & Recipe/Dish/Food & $1.2 \%$ & $0.16 \%$ \\
\hline Research & Economic/Agriculture & $0.04 \%$ & 0.01 \\
\hline Others & - & $0.01 \%$ & $0.01 \%$ \\
\hline
\end{tabular}

Table 5.2: The statistics of queries with query-categories in terms of the $\%$ of unique queries and the $\%$ of total queries. 


\subsubsection{Observations}

In our dataset, as much as $6 \%$ of the unique queries were identified as transliterated, which means that at least $40 \%$ of the words in these queries are Roman transliterations of Hindi words. The average query length for the transliterated queries is 2.86, which is less than 4.32 - the average query length of all queries. The frequency of the transliterated queries are in general less than that of the non-transliterated ones. Hence, they only constitute about $0.011 \%$ of all the queries in our dataset. However, their frequency distribution follows the same power-law pattern as the regular queries, albeit spanning mainly the medium and low frequency spectra. This also implies that a large number of transliterated and mixed queries belong to the long tail of the overall query distribution and may not have enough clickthrough data to help a search engine process them accurately. Because of this, they must be processed differently, recognizing the fact that they are rare, but together they do form a sizeable mass of the search traffic.

Table 5.2 presents the distribution statistics of the transliterated queries in each of the identified sub-categories. The numbers do not add to $100 \%$ because a small fraction of queries, $18 \%$ of unique but only $2 \%$ of all, could not be mapped to any of the categories. It is not surprising that a large fraction of the queries are NEs. Along with Websites, NEs form $50 \%$ of the unique queries, though when query frequencies are taken into account NEs only constitute $6 \%$ of all queries. Consequently, processing of transliterated NEs has received some attention from the IR researchers (Kumaran et al. 2010). Entertainment is the second largest category (27\%), of which movies and songs are the most searched categories. These queries are typically longer and more complex than NE queries, and constitute more than $32 \%$ of the transliterated query traffic. Yet, this category has hardly received any special attention from the researchers (Dua et al., 2011; Gupta et al., 2012a). We believe that Entertainment is a rich and practically important domain for MSIR, and hence we conduct our MSIR experiments on Hindi song lyrics dataset (Saha Roy et al. 2013). 


\subsection{Experiments and results}

Now we describe the experimental set up for evaluating the effectiveness of the proposed cross-view autoencoder for retrieval in mixed-script space (Gupta et al., 2014).

\subsubsection{Dataset}

We used the FIRE 2013 shared task collection on Transliterated Search Saha Roy et al., 2013) for experiments and training. The dataset comprises of a collection of documents $\left(\mathcal{D}_{1}\right)$, a queryset $(\mathcal{Q})$ and their corresponding relevance judgments. The collection contains 62,888 documents having song title and lyrics in Roman, Devanagari and mixed-scripts. Some of the Roman-script documents are in ITRANS 2 format, which is an ASCII transliteration scheme for Indic scripts. Statistics of the document collection is given in Table 5.3 (a). The $\mathcal{Q}$ contains 25 lyrics search queries for Bollywood songs in Roman script with mean query length of 4.5 words. Table 5.3 (b) lists a few examples of queries from $\mathcal{Q}$. The binary Qrels were created by manually evaluating a pool of runs generated from different systems submitted to the track. On an average, there were 47.92 relevance judgments and 6.72 relevant documents per query. The song lyrics documents were created by crawling several popular lyrics domains like dhingana, musicmaza and hindilyrix.

\begin{tabular}{|l|r|}
\hline No. of & \\
\hline Documents & 62,888 \\
Tokens & $12,738,191$ \\
Vocabulary & 135,243 \\
\hline
\end{tabular}

(a) Corpus statistics

\begin{tabular}{|l|}
\hline Sample queries \\
\hline tumse milke aisa laga \\
wah tera kya kehna \\
zindagi ke safar mein \\
\hline
\end{tabular}

(b) Example of queries

Table 5.3: Details of the dataset.

\footnotetext{
${ }^{2}$ http://en.wikipedia.org/wiki/ITRANS
} 


\subsubsection{Experimental setup}

The experimental setup is a standard ad-hoc retrieval setting. The document collection is first indexed to create an inverted index and the index lexicon is used as mining lexicon. Being this a lyrics retrieval set up, the sequential information among the terms is crucial for effectiveness evaluation, e.g. "love me baby" and "baby love me" are completely different songs. In order to capture the word-ordering, we consider word 2-grams as a unit for indexing and retrieval.

The non-trivial part of MSIR is query-expansion to handle the challenges described in Sec. 5.1.4. In order to enrich the query with equivalents, we find the equivalents of the query terms as described in Section 4.1.4 and the word 2-gram query is formulated as shown in Table 5.4. The code for the CAE is publicly available at: http://www.dsic.upv.es/ pgupta/mixed-script-ir.html

\begin{tabular}{|l|l|}
\hline Original query & ik din ayega \\
\hline $\begin{array}{l}\text { Variants of ik } \\
\text { Variants of din } \\
\text { Variants of ayega }\end{array}$ & $\begin{array}{l}\text { ik, ikk, एक, इक } \\
\text { din, didn, diin, दिन } \\
\text { आएग, ayegaa, आयेगा, }\end{array}$ \\
\hline Formulated query* & $\begin{array}{l}\text { ik\$din, ik\$didn, } \\
\text { ik\$din, ikk\$din, } \\
\text { ikk\$didn, ikk\$diin, } \\
\text { din\$ayega, din\$ayegaa, } \\
\text { didn\$ayega, } \\
\text { didn\$ayegaa, } \\
\text { diin\$ayega, } \\
\text { diin\$ayegaa, एक\$दिन, } \\
\text { इक\$दि, दिन\$आयेगा, } \\
\text { दिन\$आएगा }\end{array}$ \\
\hline
\end{tabular}

Table 5.4: Example of query formulation for transliterated search. ${ }^{*}$ Note: $\$$ and, are added for readability. 


\subsubsection{Baseline systems}

We consider a variety of baseline systems and compared them with the proposed method. The query formulation is similar for all the systems including the retrieval settings like inverted index, retrieval model and mining lexicon, except the method for finding the equivalents.

1. Naïve: The original query terms are used for the query formulation without any query-enrichment step.

2. Naïve + Trans: The original query terms and their automatic back-transliteration obtained from a commercial transliteration engin ${ }^{3}$ are used for query formulation.

3. CL-LSI: In this system, linear dimensionality reduction technique known as cross-language latent semantic indexing (Dumais et al., 1997) is used to learn the low-dimensional embedding of the terms across the scripts. Consider matrix $A_{n \times K}$ where $a_{i j}$ is the count data of $j^{\text {th }}$ feature $f_{j} \in \mathcal{F}$ in $i^{\text {th }}$ training word-pair. Such matrix $A$ is factored using CL-LSI to learn projection matrix $\left(V_{K \times m}\right)$ such that $\mathbf{h}_{q}=\mathbf{x}_{q} V$. The equivalents of the query term $t$ are obtained from 50-dimensional abstract space as described in Section 4.1.4. Thus found equivalents, along with original query terms, are used for query formulation.

4. Editex: An approximate string matching algorithm for IR proposed in Zobel and Dart (1996) is used to get equivalents of the query term. Editex uses advanced Phonix and Soundex information to normalise the pronunciation differences. The distance between such normalised strings is calculated as edit distance. Editex can handle strings only in Roman alphabet. Therefore, only Roman script equivalents of the query terms are found using Editex.

5. CCA: In this case, the problem of finding equivalents is formulated as search problem across the different views by learning hashing functions Kumar and 
Udupa, 2011). The problem of learning hash functions is formulated as a constrained minimisation problem over the training data. The training terms are represented as character bi-gram features and the learning algorithm tries to minimise the distance between similar terms in a common geometric space. In the absence of the affinity matrix (i.e., no prior information about similarity between objects is available) the learning of hash functions becomes a generalised eigenvalue formulation of the canonical correlation analysis (CCA). An inverted index of hashcodes is prepared for terms in the mining lexicon. The equivalents for the query term are found from this index according to the score given by the graph matching algorithm of Udupa and Khapra (2010a).

\subsubsection{Results and Analysis}

We evaluate the effectiveness of the proposed method, referred as CAE and compare it with all the baseline systems. The retrieval performance is measured in terms of mean average precision (MAP) and mean reciprocal rank (MRR). For each query, we evaluated the ranklist composed of the top 10 documents. The used ranking model is parameter free divergence from randomness (unsupervised DFR) as described in Amati (2006) which is shown to be suitable for short queries. The results averaged over $\mathcal{Q}$ are presented in Table 5.5. For CAE, the dimensionality selection was based on the concept of critical bottleneck dimensionality described in Chapter 7. For CL-LSI, we tried different dimensionalities in the range of $[50,200]$ with step size of 50 , but did not observe any statistical significant difference in performance. For CCA, we used the implementation from the original authors, optimised for the English-Hindi language pair. The code for CAE has been made publicly available $\AA^{4}$

The results in Table 5.5 are presented after tuning the parameter $\theta$, which is better explained later in this section. The high MRR score achieved by CAE describes its ability to fetch the first relevant document at very high ranks, which is a desirable feature for Web search in addition to better overall ranking measured by MAP.

${ }^{4}$ http://www.dsic.upv.es/ pgupta/mixed-script-ir.html 


\begin{tabular}{|l||cc||c|}
\hline Method & MRR & MAP & $\theta$ \\
\hline \hline Naïve & 0.6857 & 0.2910 & NA \\
Naïve+Trans & 0.6590 & 0.3560 & NA \\
CL-LSI & 0.7533 & 0.3522 & 0.92 \\
Editex & 0.7767 & 0.3788 & NA \\
Editex+Trans & 0.7433 & 0.4000 & NA \\
CCA & 0.7640 & 0.3891 & 0.997 \\
CAE-Mono & $\mathbf{0 . 8 0 0 0}$ & $\mathbf{0 . 4 1 5 3}$ & 0.96 \\
CAE & $\mathbf{0 . 8 7 4 0}$ & $\mathbf{0 . 5 0 3 9}$ & 0.96 \\
\hline
\end{tabular}

Table 5.5: The results of retrieval performance measured by MAP and MRR. Similarity threshold $\theta$ is tuned for best performance.

\begin{tabular}{l|cccccc}
\multicolumn{1}{l}{} & N+T & CL-LSI & Editex & CCA & Editex+T & CAE \\
\cline { 2 - 7 } Naïve & $22.5 \% / 0.09$ & $21 \% / 0.12$ & $30.1 \% / 0.03$ & $33.7 \% / 0.06$ & $37.45 \% / 0.047$ & $73.1 \% / 0.0006$ \\
N+T & - & $-0.01 \% / 0.47$ & $6.2 \% / 0.34$ & $9.1 \% / 0.27$ & $12.2 \% / 0.19$ & $41.3 \% / 0.009$ \\
CL-LSI & - & - & $7.5 \% / 0.24$ & $10.5 \% / 0.22$ & $13.57 \% / 0.12$ & $43.1 \% / 0.0004$ \\
Editex & - & - & - & $2.7 \% / 0.42$ & $5.6 \% / 0.28$ & $33.0 \% / 0.002$ \\
CCA & - & - & - & - & $2.8 \% / 0.391$ & $29.5 \% / 0.007$ \\
Editex+T & - & - & - & - & - & $26.0 \% / 0.009$ \\
\cline { 2 - 8 }
\end{tabular}

Table 5.6: The performance comparison of systems presented as $\mathrm{x} / \mathrm{y}$ where $\mathrm{x}$ denotes $\%$ increase in MAP and y denotes $p$-value according to paired significance t-test.

Although Editex is devised for English and able to operate only in the Roman script space, it performs comparably to CCA and CL-LSI. In order to make a fair comparison, we report two more configurations: CAE-Mono which considers only Roman script equivalents and Editex+Trans, in which automatic transliteration of terms are added to enrich Editex. The results clearly outline the superiority of our method for query enrichment. When compared with linear methods such as PCA and CCA, which have linear objective functions, the strong performance of CAE suggests that non-linear and non-convex objective functions are better suited for modelling terms in mixedscript spaces. A statistical comparison of methods is presented in Table 5.6. There is no significant difference in performance of Naïve+Trans, CL-LSI, Editex and CCA, while CAE significantly outperforms all the baselines, as shown with dark-gray background, which clearly shows that term equivalents found by CAE are better than 
the other methods.

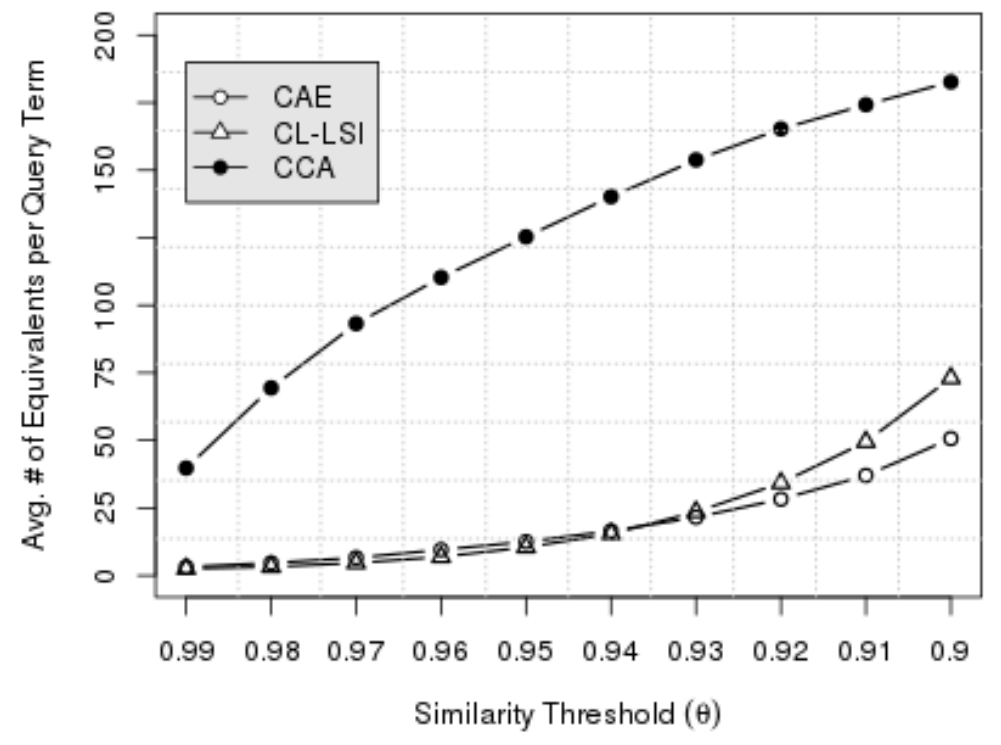

Figure 5.1: Average number of equivalents found in abstract space at similarity threshold $(\theta)$ (c.f. Section 4.1.4).

Finally, we present an analysis on the impact of $\theta$ on the resulting number of equivalents, which is directly related to the query latency. Fig. 5.1 depicts the average number of equivalents for each query term with respect to corresponding $\theta$. As can be noticed in Fig. 5.1, CCA shows a steep increase in number of equivalents. This suggests that CCA has a very dense population in the abstract space and, therefore, has around $\sim 40$ equivalents even at a strict threshold of 0.99. On the other hand, CAE and CL-LSI show a moderate increase in the number of equivalents with respect to $\theta$ value.

The effect of $\theta$ on the retrieval performance is shown in Fig. 5.2, where the parameter sweep for $\theta$ is [0.99-0.90] with step of 0.01. CAE exhibits the best performance throughout the tuning range. For CCA we also considered $\theta$ between [0.999-0.99] with step size of 0.001 to better capture its peak performance as shown in Fig. 5.2 with CCA*

We illustrate the potential of CAE for finding equivalents by showing a snippet 


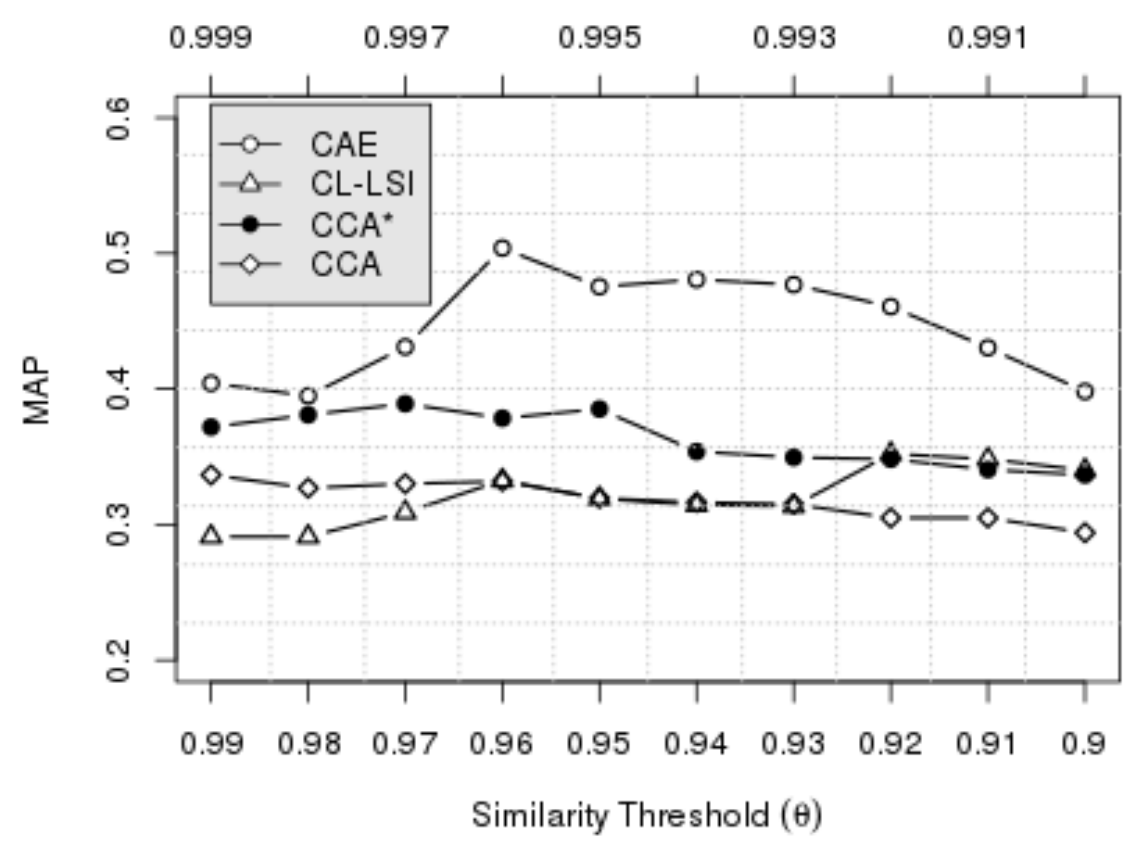

Figure 5.2: Impact of similarity threshold $(\theta)$ on retrieval performance. CCA* follows the ceiling $\mathrm{X}$-axis range [0.999-0.99].

of $20 \mathrm{D}$ abstract space as a 2D-view in Fig. 5.3. It can be noticed that mixed-script equivalents of the terms are very close to each other in small clusters and such clusters are well separated from each other. The $2 \mathrm{D}$ representation is achieved using the $\mathrm{t}$ SNE algorithm 5 . We show equivalents of a few terms found using CAE with $\theta=0.96$ in Table 5.7. The category "not sure" depicts the cases where the terms are quite close to the desired term but not correct, which may be due to a typo e.g. ehaas vs. ehsaas where the former is not a valid Hindi word.

\subsubsection{Scalability}

Among the two steps involved in finding equivalents listed in Sec. 4.1.4, the indexing step, being one-time and offline, is not a major concern. On the contrary, the real time similarity estimation during the online step while searching for equivalents is

\footnotetext{
${ }^{5}$ http: //homepage.tudelft.nl/19j49/t-SNE.html
} 


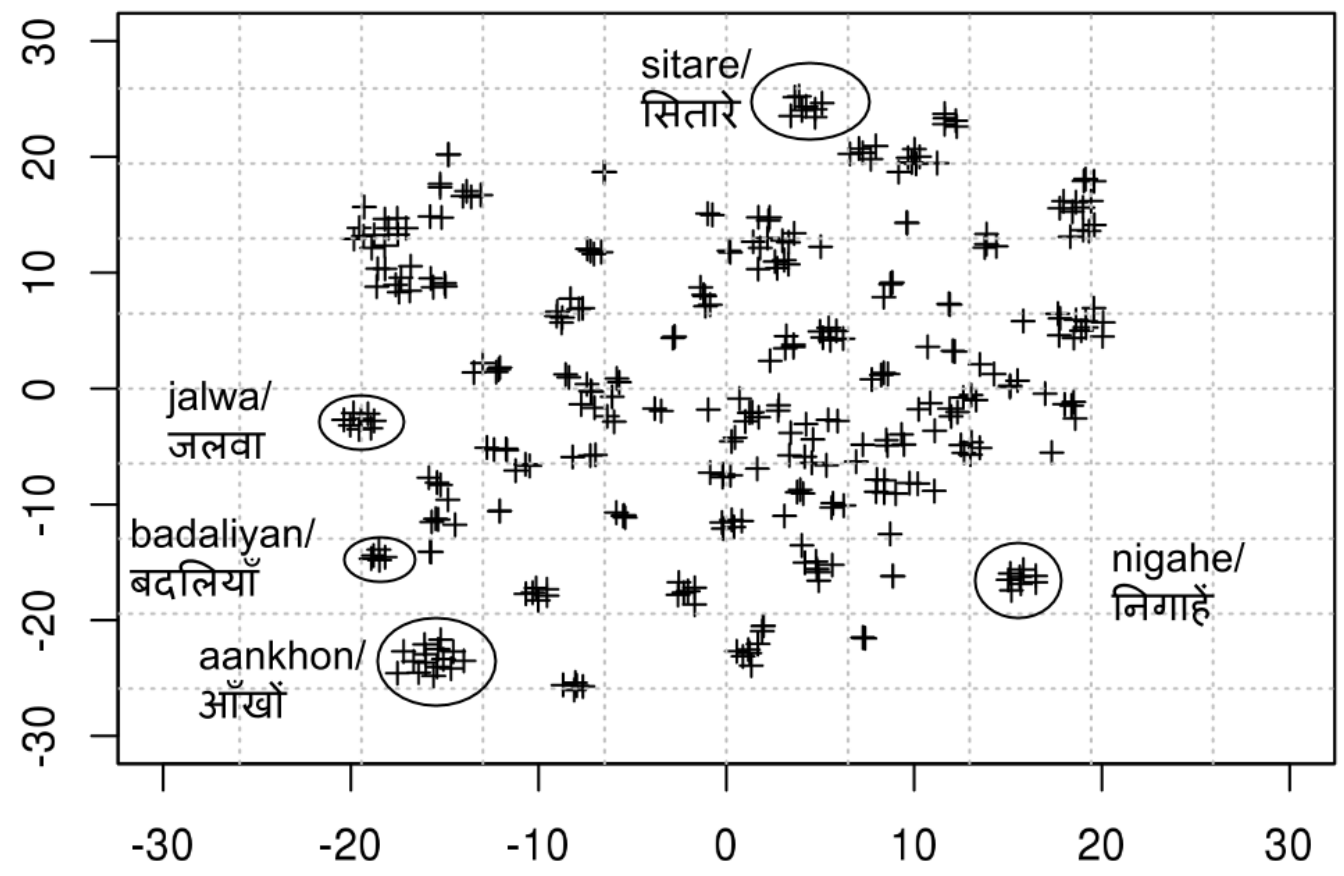

Figure 5.3: Snippet of mining lexicon projected in abstract space using CAE.

\begin{tabular}{|l|l|}
\hline Term & Variants \\
\hline \hline ehsaas & ehsas, ehasas, ehsaass, एहसास, \\
& ehasaas, ? ehaas, ehsaaas \\
mujhe & muhjhe, !mujhme, ?mujhea, मुझे, \\
& !mujheme, mujhee, muhje, muujhe, \\
& !मुझमें \\
bawra & bawara, baawra, bavra, !बरवा, \\
& bawaraa, baawara, baavra, बावरा, \\
& barava, !बिरवा \\
pe & पे, !परे, pee, !ऊपे, ?पए \\
\hline
\end{tabular}

Table 5.7: Examples of the variants extracted using CAE with similarity threshold 0.96 (words beginning with! and? mean "wrong" and "not sure" respectively).

very crucial for timely retrieval. As the similarity estimation step is essentially a matrix multiplication operation, it can be easily parallelised using multi-core CPUs or GPUs. In our case, the size of the mining lexicon was $n=135,243$ and the abstract 
space dimensionality was $m=20$. Using a multi-threading framework for matrix multiplication under normal CPU load, it takes on average 0.238 second® $\S^{6}$ for step (ii) to find equivalents for each query word. The time taken is directly proportional to the mining lexicon size $n$, dimensionality $m$ and the number of CPU/GPU cores.

\footnotetext{
${ }^{6}$ We used Intel Xeon CPU E5520 @ 2.27GHz with 4 cores, 8 processors and 12GiB memory.
} 



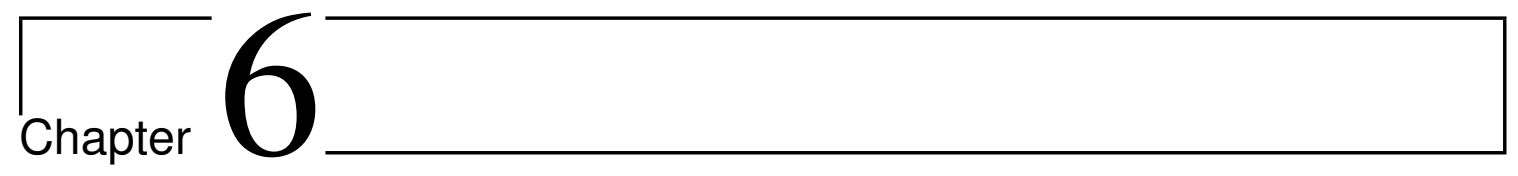

\section{Cross-language information retrieval}

Cross-language information retrieval refers to the scenario of accepting information need in one language and retrieving relevant information in a different language. An information need can be in form of a document, a natural language question or simply a search query.

This chapter aims at applying the CAE (c.f. Section 4.1) and XCNN (c.f. Section 4.2 models to various task of cross-language information retrieval. First, we give an overview of a set of existing cross-language text similarity assessment strategies and then explain a few models that are used in this dissertation in Section 6.1. The problem statement, experimental setup and results for different CLIR tasks are presented in the successive sections: cross-language plagiarism detection (Section 6.2), cross-language ad-hoc retrieval (Section 6.3), parallel sentence retrieval (Section 6.4 and source sentence retrieval for machine translation (Section 6.5). 


\subsection{Cross-language text similarity}

In general, cross-language text similarity methods can be categorised into following six categories.

(i) Lexical-based systems rely on vocabulary similarity (e.g. English-French) and linguistic influence (e.g. English computer $\rightarrow$ Spanish computadora) between languages. Similarities across words in different languages can be reflected when composing short terms; e.g. character $n$-grams or prefixes. Probably two of the first similarity models of this kind are cognateness - based on prefixes and other tokens - (Simard et al. 1992) and dot-plot - based on character 4-grams (Church, 1993). While originally proposed to align bitexts, these models are useful to measure similarity across languages (Potthast et al., 2011), but still with some limitations (BarrónCedeño et al., 2010).

(ii) Thesauri-based systems map words or concepts, such as named entities, into a common representation space by means of a multilingual thesaurus, such as Eurovoc (Steinberger et al., 2002; Gupta et al., 2012b) or EuroWordnet (Vossen, 1998). However, multilingual thesauri are not always a viable solution. For instance, Ceska et al. (2008) found that the incompleteness of the thesaurus (in that case EuroWordnet) may limit the detection capabilities. Multilingual semantic network - BabelNet (Navigli and Ponzetto, 2012) based cross-language knowledge graph analysis (CL-KGA) provides a framework to estimate these similarities in the graph space (Franco-Salvador et al., 2013).

(iii) Comparable corpus-based systems are trained over comparable corpora. One example is cross-language explicit semantic analysis (Potthast et al., 2008). Given documents $\left(d_{q}\right.$ and $\left.d^{\prime}\right)$ are represented by a vector of similarities to the documents of a so-called CL index collection $C_{I}$, i.e., $\overrightarrow{d_{q}}=\left\{\operatorname{sim}\left(d_{q}, c_{1}\right), \ldots \operatorname{sim}\left(d_{q}, c_{I}\right)\right\}$, $\overrightarrow{d^{\prime}}=\left\{\operatorname{sim}\left(d^{\prime}, c_{1}^{\prime}\right), \ldots \operatorname{sim}\left(d^{\prime}, c_{I}^{\prime}\right)\right\}$, where, $\left(c_{i} \in L\right.$ and $c_{i}^{\prime} \in L^{\prime}$ are comparable docu- 
ments). Here, sim is a monolingual similarity model, such as the cosine measure. $\vec{d}_{q}$ and $\overrightarrow{d^{\prime}}$ are then compared to compute $\operatorname{sim}\left(d_{q}, d^{\prime}\right)$.

(iv) Parallel corpus-based systems are trained on parallel corpora, either to find cross-language co-occurrences (Littman et al., 1998) or to obtain translation modules. The principles and resources of machine translation are used, but no actual translations are computed. Cross-language alignment-based similarity analysis is one of such models and it is discussed in more detail in Section 6.1.3.

(v) Machine translation-based systems simplify the problem by turning it into a monolingual problem. The main approach is as follows: $(i)$ a language detector is applied to determine the most likely language of the documents at hand; (ii) if not written in the comparison language, one of the documents is translated; and (iii) a monolingual comparison is carried out between the two documents.

(vi) Translingual continuous space systems learn continuous space representation for text across languages and measure similarity in this space. Models such as cross-language latent semantic indexing (Dumais et al., 1997) and oriented principle component analysis (Platt et al. 2010 ) learn linear projections for text through matrix factorization methods. While S2Net (Yih et al., 2011) uses a Siamese neural network to learn the projections. The models proposed in this thesis, CAE and $\mathrm{XCNN}$, also fall in this category.

Now, we present a few models that can be used for cross-language text similarity.

\subsubsection{Cross-language character $n$-grams (CL-CNG)}

Cross-language character $n$-grams was originally proposed by Mcnamee and Mayfield (2004) for cross-language information retrieval. It is a very simple model that decomposes the text from two language sources into smaller units such as character n-grams. Standard normalisation techniques are applied such as lower-casing 
and diacritics removal. Following Potthast et al. (2011), we used $n=3$ for our experiments. The similarity between text representations is computed using cosine similarity.

\subsubsection{Cross-language explicit semantic analysis (CL-ESA)}

Cross-language explicit semantic analysis (Potthast et al. 2 2008) extends the explicit semantic analysis model (Gabrilovich and Markovitch, 2007) to work in a crosslanguage scenario. This model represents each text by means of its similarities with a document collection $D$. Even though the indexing with $D$ is performed at monolingual level, using a multilingual document collections with comparable documents across languages, e.g. Wikipedin allows for the resulting vectors from different languages to be compared. Formally, having a matrix $D_{L}$ where rows represent documents of a collection in a language $L$, a document $d_{L}$ is indexed as follows:

$$
d_{D_{L}}=D_{L} \cdot d_{L}^{T}
$$

where $d_{D_{L}}$ denotes the resulting indexed vector of document $d_{L}$ in $D_{L}$. Documents represented in $d_{L}$ and $D_{L}$ use a vector representation such as VSM with term frequency-inverse document frequency (TF-IDF) weighting (Salton et al., 1983). The similarity between two documents $d_{L}$ and $d_{L^{\prime}}^{\prime}$ is estimated as $\varphi\left(d_{D_{L}}, d_{D_{L^{\prime}}}^{\prime}\right)$, where $\varphi$ is a vector similarity function, and $D_{L}$ and $D_{L^{\prime}}$ are comparable document collections between $L$ and $L^{\prime}$.

\subsubsection{Cross-language alignment-based similarity analysis (CL- ASA)}

Cross-language alignment-based similarity analysis (Barrón-Cedeño et al., 2008) measures the similarity between two documents by on the lines of the Bayes's rule for

\footnotetext{
1 https://es.wikipedia.org/
} 
machine translation - composition of language model and translation model. It computes the likelihood of $d^{\prime}$ to be a translation of $d$ as shown in Eq. 6.2.

$$
\mathrm{S}\left(d, d^{\prime}\right)=\varrho\left(d^{\prime}\right) \mathrm{p}\left(d \mid d^{\prime}\right) .
$$

CL-ASA uses $\varrho\left(d^{\prime}\right)$ component as length model which captures the translation length factor as defined in (Pouliquen et al., 2003). The translation model depicted by conditional probability $\mathrm{p}\left(d \mid d^{\prime}\right)$ in Eq. 6.2 is replaced by a statistical bilingual dictionary score and computed as shown in Eq. 6.3.

$$
\rho\left(d \mid d^{\prime}\right)=\sum_{x \in d} \sum_{y \in d^{\prime}} \mathrm{p}(x, y),
$$

where $\rho\left(d \mid d^{\prime}\right)$ no longer represents a probability measure and the dictionary $\mathrm{p}(x, y)$ defines the likelihood of word $x$ of being a valid translation of $y$. The CL-ASA model is trained according to the parameters reported in Barrón-Cedeño et al. (2013).

\subsubsection{Cross-language knowledge graph analysis (CL-KGA)}

Cross-language knowledge graph analysis (Franco-Salvador et al., 2013; Franco-Salvador et al. 2016) represents documents in a semantic graph space by means of knowledge graphs. A knowledge graph is created as a subset of a multilingual semantic network, e.g. BabelNet (Navigli and Ponzetto, 2012), focused on the concepts belonging to a text. As stated in Franco-Salvador et al. (2016), these graphs have several interesting characteristics that can be exploited for cross-language similarity estimation. Note, for instance, that concepts are represented in BabelNet by means of multilingual sets of synonyms. Therefore, knowledge graphs created from documents in different languages can be directly compared. Formally, having a pair of graphs $\left(G, G^{\prime}\right), G \in d_{L}$ and $G^{\prime} \in d_{L^{\prime}}^{\prime}$, the similarity $S_{g}\left(G, G^{\prime}\right)$ between them can be estimated for concepts and relations independently from each other. The similarity between the concepts is calculated using the Dice's coefficient (Jackson et al., 1989): 


$$
\mathrm{S}_{c}\left(G, G^{\prime}\right)=\frac{2 \cdot \sum_{c \in \mathrm{V}(G) \cap \mathrm{V}\left(G^{\prime}\right)} \mathrm{w}(c)}{\sum_{c \in \mathrm{V}(G)} \mathrm{w}(c)+\sum_{c \in \mathrm{V}\left(G^{\prime}\right)} \mathrm{w}(c)},
$$

where $\mathrm{V}(G)$ is the set of concepts in the graph and $\mathrm{w}(c)$ is the weight of a concept c. Likewise, the similarity between the relations is calculated as:

$$
\mathrm{S}_{r}\left(G, G^{\prime}\right)=\frac{2 \cdot \sum_{r \in \mathrm{E}(G) \cap \mathrm{E}\left(G^{\prime}\right)} \mathrm{w}(r)}{\sum_{r \in \mathrm{E}(G)} \mathrm{w}(r)+\sum_{r \in \mathrm{E}\left(G^{\prime}\right)} \mathrm{w}(r)},
$$

where $\mathrm{E}(G)$ is the set of relations in the graph and $\mathrm{w}(r)$ is the weight of a semantic relation $r$. Finally, the two above measures of conceptual $\left(\mathrm{S}_{c}\right)$ and relational $\left(\mathrm{S}_{r}\right)$ similarity are interpolated to obtain an integrated measure $\mathrm{S}_{g}\left(G, G^{\prime}\right)$ between knowledge graphs:

$$
\mathrm{S}_{g}\left(G, G^{\prime}\right)=a \cdot \mathrm{S}_{c}\left(G, G^{\prime}\right)+b \cdot \mathrm{S}_{r}\left(G, G^{\prime}\right),
$$

where $a$ and $b$ (with $a+b=1$ ) are the parameters depending on the relevance of concepts and relations respectively $!^{2}$

Concepts are weighted using their graph outdegree (Navigli and Ponzetto, 2012). In contrast, relations are weighted using the original weights between relations provided in BabelNet. These weights were calculated using an extension of the extended gloss overlap measure (Banerjee and Pedersen, 2003) which weights semantic relations between WordNet (Fellbaum, 1998) and Wikipedia concepts. For more details about the CL-KGA model please refer to the original works from (Franco-Salvador et al. 2013; Franco-Salvador et al., 2016).

\footnotetext{
${ }^{2}$ In this work we used the optimal values provided in Franco-Salvador et al. (2016) for concepts and relations: $a=b=0.5$.
} 


\subsubsection{Cross-language latent semantic indexing (CL-LSI)}

CL-LSI is cross-language extension of latent semantic indexing, which performs the singular value decomposition (SVD) of a document-term matrix D (Dumais et al. 1997). The matrix $\mathbf{D}$ is constructed from a parallel corpus, where each parallel counterparts are concatenated as shown in Fig. 6.1. A standard approach to constructing D is using $\log (\mathrm{TF})$-IDF weighting scheme as shown in Eq. 6.7.

$$
D_{i j}=\log _{2}\left(1+\mathrm{tf}_{i j}\right) * \log \left(\frac{n}{d_{j}}\right)
$$

where, $\mathrm{tf}_{i j}$ represents frequency of term $j$ in document $i, n$ is the total number of documents in the collection and $d_{j}$ represents document frequency of term $j$.

CL-LSI obtains a decomposition of $\mathbf{D}$ into the so called singular vectors and singular values. The top $k$ singular vectors or, principal components, of $\mathbf{D}$ form a projection space (as shown in Eq. 6.8) in which documents can be compared on a semantic basis. This decomposition, know as SVD, factorizes D into three matrices - an $m \times r$ term-concept vector matrix $U$, an $r \times r$ singular values matrix $\Sigma$, and a $n \times r$ document-concept vector matrix $V$ where $r$ is the rank of the matrix, i.e. $r \leq \min (m, n)$. Then, the resulting decomposition is reduced to rank $k \ll r$ keeping only the $k$ largest principal components. The inherent idea is that semantically similar terms across languages will be mapped into space representations that are closer to each other. According to this, semantically similar documents will appear close to each other in the reduced comparison space.

$$
\begin{aligned}
\mathbf{D} & =U \Sigma V^{T} \\
\mathbf{D} & \approx \mathbf{D}_{k} \\
\mathbf{D}_{k} & =U_{k} \Sigma_{k} V_{k}^{T}
\end{aligned}
$$

A text fragment is represented as $\vec{y}$ in the latent space as shown in Eq. 6.9. 


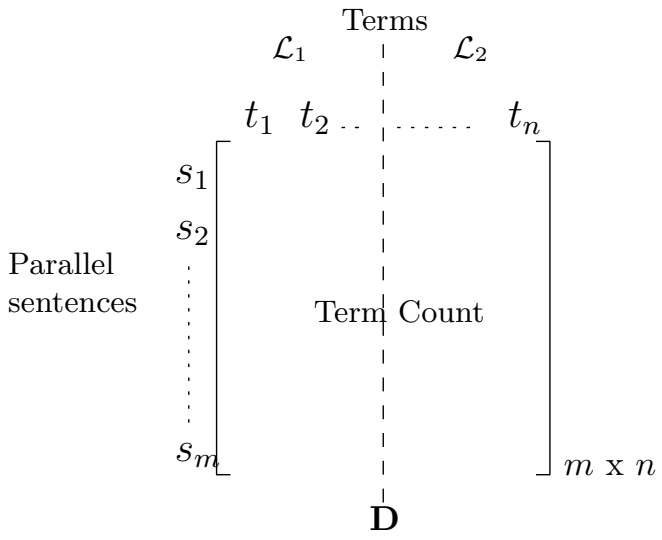

Figure 6.1: Document-term matrix formulated from a parallel sentences corpus.

$$
\vec{y}=\vec{x} * V
$$

where $\vec{x}$ is vector space representation of text fragment with TF-IDF weighting scheme (as shown in Eq. 6.7) and $V$ is document-concept vector matrix.

\subsubsection{Oriented principal component analysis (OPCA)}

OPCA extends CL-LSI and formulates the problem in a more extended way by introducing a noise component. It solves the generalised eigenproblem, which maximises the signal-to-noise ratio (Platt et al. 2010$)$ :

$$
\mathbf{S} v_{j}=\lambda_{j} \mathbf{N} v_{j}
$$

where, $\mathbf{S}$ is the covariance matrix of the documents in different languages and $\mathbf{N}$ is the covariance matrix of the differences among parallel documents which are considered noise.

Specifically, OPCA creates a weighted document-term matrix $\mathbf{D}_{m}$ for each language, where $m \in\{1,2\}$ for a cross-language case. The signal covariance matrix $\mathbf{S}$, is defined as follows: 


$$
\mathbf{S}=\sum_{m} \frac{\mathbf{D}_{m}^{T} \mathbf{D}_{m}}{n}-\vec{\mu}^{T} \vec{\mu}
$$

where, $\vec{\mu}$ is the mean of each $\mathbf{D}_{m}$ over its columns. In order to make each $\mathbf{D}_{m}$ of equal size, their columns refer to the total vocabulary inclusive of all languages.

The noise covariance matrix $\mathbf{N}$, is defined as follwos:

$$
\mathbf{N}=\sum_{m} \frac{\left(\mathbf{D}_{m}-\mathbf{D}\right)^{T}\left(\mathbf{D}_{m}-\mathbf{D}\right)}{n}+\gamma \mathbf{I}
$$

where, $\mathbf{D}$ is the mean across all language:

$$
D=\frac{1}{M} \sum_{m} \mathbf{D}_{m}
$$

The term $\gamma \mathbf{I}$ acts as a regularisation term.

Theoretically, OPCA tries to minimise the distance between the parallel documents at the same time of maximising the overall variance of the data. The overall variance of the data refers to variance among different non-parallel documents. The parameters of OPCA are tuned according to Platt et al. (2010).

\subsubsection{Similarity learning via siamese neural network (S2Net)}

Following the general Siamese neural network architecture (Bromley et al., 1993), S2Net trains two identical neural networks concurrently. The S2Net takes in parallel data with binary or real-valued similarity score and updates the model parameters accordingly (Yih et al. 2011). It optimises a dynamic objective function which is directly modelled by using cosine similarity. The projection operation can be described as follows:

$$
y_{d}=W * x_{d}
$$


where, $x_{d}$ is the input term vector for document $d, W$ is the learnt projection matrix (represented by the model parameters) and $y_{d}$ is the latent representation of document $d$. The parameters of the S2Net are tuned according to the details provided in Yih et al. (2011).

\subsubsection{Machine translation}

Given a source string $s_{1}^{J}=s_{1} \ldots s_{j} \ldots s_{J}$ to be translated into a target string $t_{1}^{I}=t_{1} \ldots t_{i} \ldots t_{I}$, a phrase-based statistical MT system aims at choosing, among all possible target strings, the string that maximises the conditional probability:

$$
\tilde{t}_{1}^{I}=\underset{t_{1}^{I}}{\operatorname{argmax}} P\left(t_{1}^{I} \mid s_{1}^{J}\right)
$$

where $I$ and $J$ are the number of words in the target and source sentences, respectively.

The phrase-based system segments the source sentence into phrases, then translates each phrase by using bilingual dictionary (also referred to as translation table) containing source and target phrase pairs and their estimated probabilities $\left(s_{1} . . s_{n}|| \mid t_{1} . . t_{m}\right)$. Incrementally, the system composes the target sentence by exploring different combinations of phrase pairs. Standard implementations of the phrase-based system use several features, or probabilistic models, to estimate the overall translation probability in Eq. 6.15. The most common features used by phrase-based translation systems include: relative frequencies, target language model, word and phrase bonus, sourceto-target and target-to-source lexical models, and reordering model (Koehn et al. 2007).

In the translation-based approach to CLIR, we train a phrase-based machine translation system to perform query translations. The translation system is trained on domain-related parallel data using the standard state-of-the-art Moses toolkit3 with default parameters (Koehn et al. 2007). For the CLIR system implementation,

\footnotetext{
$\sqrt[3]{\text { http://www.statmt.org/moses/ }}$
} 
the query is translated into the language of the document collection and then similarity is calculated using the BM25 measure in a monolingual setting Although we consider this system to be a baseline, we do not expect all cross-language latent approaches to necessarily outperform it, because this system operates in a monolingual full-dimensional vector space in contrast to latent semantic models, which operate in a cross-language low dimensional abstract space.

\subsubsection{Hybrid models}

The knowledge-based similarity analysis (KBSim) model (Franco-Salvador et al. 2014) extends CL-KGA in order to combine the benefits of both, the knowledge graph and the multilingual vector-based representations. Key to this approach is the weighted combination of these two representations according to the relevance of the knowledge graphs. This allows to increase the contribution of the multilingual vector-based representations in case of non-informative knowledge graphs. Given a source document $d$ and a target document $d^{\prime}$, we calculate the similarities between the respective knowledge graphs and multilingual vector representations, and combine the two resulting similarities to obtain a knowledge-based similarity as follows:

$$
\mathrm{S}\left(d, d^{\prime}\right)=\alpha * \mathrm{~S}_{g}\left(G, G^{\prime}\right)+(1-\alpha) * \mathrm{~S}_{v}\left(\vec{v}, \vec{v}^{\prime}\right),
$$

where $\mathrm{S}_{g}\left(G, G^{\prime}\right)$ is the knowledge graph similarity of Eq. 6.6, $\mathrm{S}_{v}\left(\vec{v}, \vec{v}^{\prime}\right)$ is the vectorbased similarity, and $\alpha$ is an interpolation factor that is calculated as the edge density of the knowledge graph $G$ :

$$
\alpha=\frac{|\mathrm{E}(G)|}{|\mathrm{V}(G)|(|\mathrm{V}(G)|-1)}
$$

Note that, by using the factor $\alpha$ to interpolate the two similarities in Eq. 6.16, the relative importance of each model is determined.

\footnotetext{
${ }^{4}$ We tried different retrieval models like BM25 and divergence from randomness based but the difference in performance was not statistically significant
} 
The vector-based similarity $\mathrm{S}_{v}\left(\vec{v}, \vec{v}^{\prime}\right)$ computes the cosine similarity between vectors in the continuous space. We adopt this framework to include information provided by the continuous representation into similarity estimation. We evaluate the performance of KBSim (S2Net), KBSim (CAE) and KBSim (XCNN) in Section 6.2.4 for cross-language plagiarism detection task.

\subsubsection{Continuous word alignment-based similarity analysis (CWASA)}

The aforementioned models allow for learning a real-valued continuous space representation of texts. All of them combine basic word level representations by summing over terms in order to model sequences of words. The method presented in this section provides an alternative way to combine word level vectors by means of alignments to represent text. The continuous word alignment-based similarity analysis model is based on the text-to-text relatedness proposed by (Hassan and Mihalcea, 2011). It estimates the similarity between documents by efficiently aligning their continuous word representations using directed edges. Formally, the similarity $\mathrm{S}\left(d, d^{\prime}\right)$ between two documents $d$ and $d^{\prime}$ is estimated as follows:

$$
\mathrm{S}\left(d, d^{\prime}\right)=\frac{1}{|\Phi|} \sum_{c_{k} \in \Phi} c_{k},
$$

where $d=\left(x_{1}, \ldots, x_{n}\right)$ and $d^{\prime}=\left(y_{1}, \ldots, y_{m}\right)$ are represented as lists of continuous word vectors, and $\Phi$ is generated from the list $\Phi^{\prime}=\left\{c_{1}^{\prime}, \ldots, c_{n+m}^{\prime}\right\}$ that satisfies Eq. 6.19.

$$
c_{k}^{\prime}= \begin{cases}\max _{i=k, x_{i} \in d, y_{j} \in d^{\prime}} \varphi\left(x_{i}, y_{j}\right), & \text { if } k \leq n \\ \max _{j=k-n, x_{i} \in d, y_{j} \in d^{\prime}} \varphi\left(x_{i}, y_{j}\right), & \text { otherwise }\end{cases}
$$

where $1 \leq i \leq n, 1 \leq j \leq m, 1 \leq k \leq n+m, \varphi$ is the cosine similarity function, and being $\Phi=\left\{c_{1}, \ldots, c_{z} \mid \max (n, m) \leq z \leq n+m\right\}, \Phi \subseteq \Phi^{\prime}$, the set of cosine similarities 
without pairing repetition: 5 that represents the strongest semantic pairing between the continuous word representations of documents $d$ and $d^{\prime}$.

Basically, in Eq. 6.19 each word in $d$ is aligned with the closest one in $d^{\prime}$ and vice versa using directed relationships. Next, the duplicated alignments are removed, i.e., those equally aligned in both directions. Finally, the similarity score between $d$ and $d^{\prime}$ is estimated by Eq. 6.18 as the average of the different alignments. More details on CWASA can be found in (Franco-Salvador et al., 2016).

\subsection{Cross-language plagiarism detection}

Automatic plagiarism detection entails identifying plagiarised text fragments and their corresponding original source. The task is defined in Sec. 6.2.1] and also popularly used in PAN ${ }^{6}$ track on plagiarism detection at CLEF (Potthast et al. 2009). There have been many approaches to plagiarism detection (Potthast et al. 2009 , 2010, 2011; Barrón-Cedeño, 2012; Barrón-Cedeño et al., 2013; Franco-Salvador et al. 2013), but, as far as we know, latent semantic methods have not been explored for this problem yet. We believe semantic similarity assessed by means of latent features can provide a new interest approach to plagiarism detection.

\subsubsection{Problem statement}

Let $d_{q}$ be a suspicious document and $D$ a set of potential source documents. The core problem of plagiarism detection is to identify the set of all fragment pairs $\left\{s_{q}, s\right\}$ such that fragments $s_{q} \in d_{q}$ have a high chance to be borrowed from fragments $s \in d$ (with $d \in D$ ). After $\left\{s_{q}, s\right\}$ are identified, an expert can determine whether each fragment pair is indeed a case of plagiarism (no proper citation is provided). From a cross-language (CL) perspective, $d_{q} \in L$ and $d^{\prime} \in L^{\prime}$, where $L \neq L^{\prime}$, represent different languages. This problem is referred to as cross-language plagiarism detection

\footnotetext{
${ }^{5}$ The same pair of words are not allowed to be aligned twice. 6 http://pan.webis.de/
} 
(CLPD).

We follow the general framework of cross-language plagiarism detection introduced in Potthast et al. (2011). The process is divided into the three steps described below:

(i) Candidate retrieval. A set of candidate documents $D^{*}$ is retrieved from $D^{\prime}$ (with $\left|D^{*}\right| \ll\left|D^{\prime}\right|$ ). $D^{*}$ contains the most similar documents to $d_{q}$ and, therefore, the most likely to contain potential cases of re-use.

(ii) Detailed analysis. $d_{q}$ is compared against every $d^{\prime} \in D^{*}$ section-wise. If a pair $\left\{s_{q}, s^{\prime}\right\}$ is identified to be more similar than expected for independently generated texts, it is selected as a candidate of plagiarism.

(iii) Heuristic post-processing. Plagiarism candidates that are not long or do not have similarity above a threshold are discarded. Additionally, heuristics are applied to merge nearby candidates $]^{7}$

Based on this framework, most of the research done on CL similarity estimation is used for the candidate retrieval and detailed analysis steps, while heuristic postprocessing step mostly incorporates the domain knowledge for the CLPD task.

\subsubsection{Detailed analysis method}

The step of identifying plagiarised sections in suspicious document $d_{q}$ from source document $d^{\prime}$ is referred to as detailed analysis. A framework for detailed analysis is presented in Algorithm 2 which is also a contribution of this work (Barrón-Cedeño et al. 2013). In the detailed analysis, $d_{q}$ and $d^{\prime}$ are split into chunks of certain length $w$ and step size $t$. We select $w=5$ and $t=2$ sentences aiming at considering chunks close to paragraphs (Barrón-Cedeño et al., 2013); $\operatorname{sim}\left(s_{q}, s^{\prime}\right)$ computes the similarity between the text fragments based on a similarity estimation algorithm discussed

\footnotetext{
${ }^{7}$ This step had been originally intended to filter false positives, such as cases of borrowing with proper citation (Stein et al. 2007 ).
} 


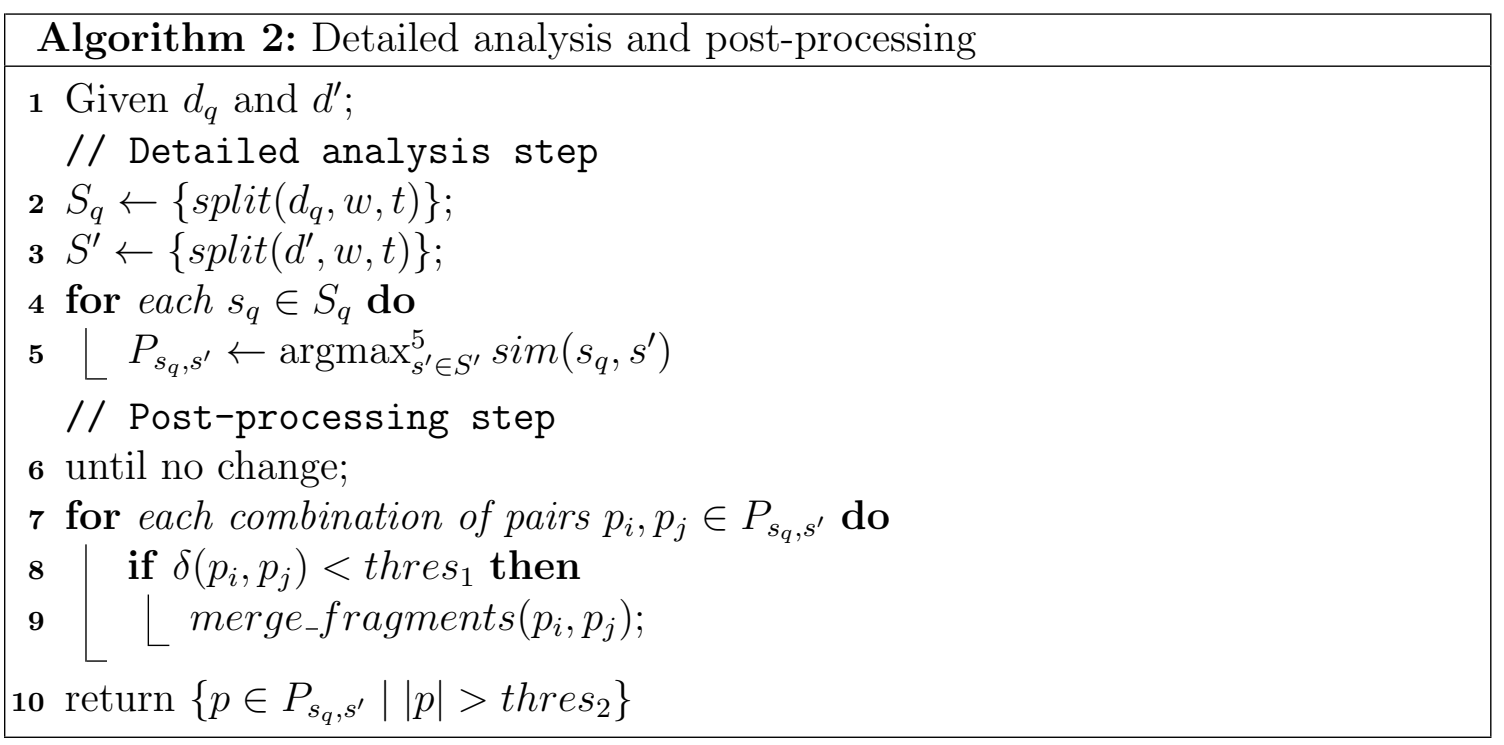

later in this section. Expression $\operatorname{argmax}_{s \in S}^{5}$ retrieves the 5 most similar fragments $s \in S$ with respect to $s_{q}$. The resulting candidate pairs $\left\{s_{q}, s\right\}$ are stored into pairset $P_{s_{q}, s^{\prime}}$, which constitutes the input for the post-processing step. If the distance in characters between two (highly similar) candidate pairs $\delta\left(p_{i}, p_{j}\right)$ is lower than a predefined threshold thres $s_{1}=1,500, p_{i}$ and $p_{j}$ are merged. Only those candidates that are composed of at least three of the identified fragments (thres $)_{2}$ ) are considered potentially plagiarised (thresholds are defined empirically). This algorithm has been used for evaluating all the models that are compared in the second experiment of Sec. 6.2.4.2. The code for this algorithm is publicly available at: https://github. com/parthg/clpd-kbs

\subsubsection{Dataset and experiments}

The experimental evaluation of cross-language plagiarism detection is carried out with the PAN-PC-11 8 dataset. It was created for the 2011 plagiarism detection competition of PAN at CLEF'? ${ }^{9}$. The dataset consists of Spanish-English (ES-EN)

8 http://www.uni-weimar.de/en/media/chairs/webis/corpora/corpus-pan-pc-11/ ${ }^{9}$ http://www.clef-initiative.eu/ 


\begin{tabular}{|c|c|c|c|}
\hline \multicolumn{2}{|c|}{ Spanish-English documents } & \multicolumn{2}{|l|}{ German-English documents } \\
\hline Suspicious & 304 & Suspicious & 251 \\
\hline Source & 202 & Source & 348 \\
\hline \multicolumn{4}{|c|}{ Plagiarism cases $\{$ Spanish,German\}-English } \\
\hline $\begin{array}{l}\text { Case length } \\
\text { - Long length cases } \\
\text { - Medium length cases } \\
\text { - Short length cases }\end{array}$ & $\begin{array}{l}1,506 \\
2,118 \\
1,951\end{array}$ & $\begin{array}{l}\text { Obfuscation } \\
\text { - Translated automatic obfuscation } \\
\text { - Translated manual obfuscation }\end{array}$ & $\begin{array}{l}5,142 \\
433\end{array}$ \\
\hline
\end{tabular}

Table 6.1: Statistics of PAN-PC-11 cross-language plagiarism detection partitions.

and German-English (DE-EN) partitions for CL plagiarism detection. The crosslanguage plagiarism cases were generated using with Google translate service ${ }^{10}$. In addition, PAN-PC-11 contains also cases of plagiarism with manual obfuscation after automatic translation which includes paraphrasing. Table 6.1 presents the statistics of the dataset.

The models are evaluated through two different experimental setup: A \& B. In the experiment $\mathrm{A}$, the whole document $d_{q}$ is plagiarised using document $d^{\prime}$ and the task is to find $d^{\prime} \in D^{\prime}$ for each $d_{q}$. This setting aims at assessing the power of models for candidate retrieval. The performance for this experiment is measured in terms of Recall at position $k(R @ k)$ where $k=\{1,5,10,20\}$. In the experiment $\mathrm{B}$, for given $d_{q}$ and $d^{\prime}$ the task is to find the plagiarism fragments of $d_{q}$ from $d^{\prime}$. This setting aims at assessing the power of models for the detailed analysis step. The performance of experiment $\mathrm{B}$ is evaluated in terms of the standard plagiarism detection metrics in the PAN shared task: precision, recall, granularity, and plagdet (Potthast et al. 2010), described below.

Let $S$ denote the set of plagiarism cases in the suspicious documents, and let $R$ denote the set of plagiarism detections that the detector reports for these documents. A plagiarism case $s \in S$ is represented by the subset of contiguous characters that forms it, which is defined in terms of offsets with respect to the beginning of the document. Likewise, $r \in R$ represents a plagiarism detection. Based on these repres-

\footnotetext{
10 https://translate.google.com/
} 
entations, the precision and the recall at character level of $R$ under $S$ are measured as follows:

$$
\begin{aligned}
& \operatorname{precision}(S, R)=\frac{1}{|R|} \sum_{r \in R} \frac{\left|\bigcup_{s \in S}(s \sqcap r)\right|}{|r|} ; \\
& \operatorname{recall}(S, R)=\frac{1}{|S|} \sum_{s \in S} \frac{\left|\bigcup_{r \in R}(s \sqcap r)\right|}{|s|},
\end{aligned}
$$

where $s \sqcap r=s \cap r$ if $r$ detects $s$ and $\emptyset$ otherwise. Note that these definitions of precision and recall do not account for the fact that plagiarism detectors sometimes report overlapping or multiple detections for a single plagiarism case. To address this issue, we also measured the detector's granularity:

$$
\operatorname{granularity}(S, R)=\frac{1}{\left|S_{R}\right|} \sum_{s \in S_{R}}\left|R_{s}\right|,
$$

where $S_{R} \subseteq S$ are cases detected by detectors in $R$, and $R_{s} \subseteq R$ are detections of $S$, i.e., $S_{R}=\{s \mid s \in S \wedge \exists r \in R: r$ detects $s\}$ and $R_{s}=\{r \mid r \in R \wedge r$ detects $s\}$. Granularity can take on value larger than one which indicates one plagiarism case is identified in multiple parts, which is not ideal. The three previous metrics can be combined in order to obtain an overall score for plagiarism detection, which is referred to as plagdet:

$$
\operatorname{plagdet}(S, R)=\frac{F_{1}(S, R)}{\log _{2}(1+\operatorname{granularity}(S, R))} .
$$

where $F_{1}$ is harmonic mean of precision and recall, popularly known as $F_{1}$ score as described below:

$$
F_{1}=2 * \frac{\text { precision } * \text { recall }}{\text { precision }+ \text { recall }}
$$




\subsubsection{Results and analysis}

In this section we present the results and the corresponding analysis for the experimental evaluation on cross-language plagiarism detection. First, details on specific experimental settings for each model are presented, followed by the experiments and their corresponding results.

(i) CL-C3G It is CL-CNG using character 3-grams, as recommended in Potthast et al. (2011).

(ii) CL-ESA We used 10,000 Spanish-German-English comparable Wikipedia pages as document collection. All pages contain more than 10,000 characters and were represented using TF-IDF weighting. The similarities are computed using the cosine similarity and the IDF of the words is calculated from the complete Wikipedia in each language.

(iii) CL-ASA We used a statistical dictionary trained using the word-alignment model IBM-1 Och and Ney, 2003) on the JRC-Acquis corpus (Steinberger et al. 2006).

(iv) CL-KGA We used the multilingual semantic network BabelNet (Navigli and Ponzetto, 2012) to construct the graph and parameter tuning is as per (FrancoSalvador et al. 2013).

(v) S2Net, CAE, XCNN We trained these models as described in Section 6.3.2. We present experimental results for each of these models alone and in two different settings: $(i)$ when CWASA composition model is applied on embeddings learnt through continuous models; and (ii) hybrid models using the KBSim framework.

For the readability and ease of comparison of the results, the models are grouped according to their category: (a) vector space approaches, (b) continuous space mod- 


\begin{tabular}{|c|c|c|c|c|c|c|c|c|c|}
\hline \multirow{2}{*}{\multicolumn{2}{|c|}{ Model }} & \multicolumn{4}{|c|}{ Spanish-English } & \multicolumn{4}{|c|}{ German-English } \\
\hline & & R@1 & R@5 & $R @ 10$ & R@20 & $\mathrm{R@1}$ & R@5 & $R @ 10$ & $\mathrm{R} @ 20$ \\
\hline & CL-KGA & 0.917 & 0.946 & 0.956 & 0.961 & 0.786 & 0.865 & 0.893 & 0.911 \\
\hline & CL-ASA & 0.663 & 0.787 & 0.819 & 0.853 & 0.523 & 0.693 & 0.755 & 0.806 \\
\hline & CL-ESA & 0.677 & 0.784 & 0.824 & 0.858 & 0.481 & 0.611 & 0.666 & 0.720 \\
\hline & CL-C3G & 0.497 & 0.672 & 0.743 & 0.805 & 0.204 & 0.393 & 0.489 & 0.593 \\
\hline \multirow[t]{3}{*}{ (b) } & S2Net & 0.637 & 0.763 & 0.809 & 0.852 & 0.508 & 0.675 & 0.744 & 0.799 \\
\hline & $\mathrm{XCNN}$ & 0.468 & 0.648 & 0.721 & 0.786 & 0.362 & 0.561 & 0.647 & 0.728 \\
\hline & CAE & 0.509 & 0.717 & 0.784 & 0.836 & 0.308 & 0.513 & 0.607 & 0.697 \\
\hline \multirow[t]{3}{*}{ (c) } & CWASA (XCNN) & 0.881 & 0.921 & 0.937 & 0.946 & 0.739 & 0.823 & 0.849 & 0.873 \\
\hline & CWASA (S2Net) & 0.859 & 0.909 & 0.921 & 0.936 & 0.601 & 0.731 & 0.779 & 0.818 \\
\hline & CWASA $(\mathrm{CA}$ & 0.536 & 0.695 & 0.754 & 0.803 & 0.543 & 0.701 & 0.760 & 0.806 \\
\hline \multirow[t]{3}{*}{ (d) } & KBSim (S2Net) & 0.920 & 0.949 & 0.956 & 0.961 & 0.809 & 0.878 & 0.901 & 0.921 \\
\hline & KBSim & 0.917 & 0.945 & 0.956 & 0.962 & 0.791 & 0.870 & 0.893 & 0.911 \\
\hline & KBSim (XCNN) & 0.858 & 0.907 & 0.924 & 0.935 & 0.741 & 0.843 & 0.872 & 0.897 \\
\hline
\end{tabular}

Table 6.2: $\quad$ ES-EN and DE-EN performance analysis in terms of R@ $k$, where $k=\{1,5$, $10,20\}$. Best results within each category are highlighted in bold-face.

els, (c) continuous space models with CWASA composition, and (d) model combinations using KBSim.

\subsubsection{Experiment A: Cross-language similarity ranking}

In this experiment, the models are evaluated using $\mathrm{R} @ k$, which captures the recall of plagiarism cases within $k$ positions in the rank-list. The results for ES-EN and DE-EN language pairs are presented in Table 6.2. In general, results for DE-EN are lower than its ES-EN counterpart but the overall ranking of the models does not change. The coverage of the vocabulary is calculated by finding the average number of words in a document present in the vocabulary, and averaged over the corpus. English has around $82 \%$ of coverage and Spanish and German have $72 \%$ and $42 \%$, respectively. Low word coverage in German is mainly due to its agglutinative nature and justifies the overall low results for German.

Compared to vector space models in group (a), the continuous space models of group (b) offered sub-optimal performance. Among group (b), the S2Net obtained 
the best results. It should be noted that S2Net and CAE directly learn representations of text using the bag-of-words approach. In contrast, XCNN learns word-level embeddings and hence when modeling documents, which are large fragments of text ( $\sim 1000$ words), the summation of such a large number of word-level representations affects the discriminative power of the model, affecting XCNN performance. The advantages of XCNN will be more clear in Experiment B, where the fragments being compared are small and this effect is less severe.

The use of a different composition method with continuous representation, such as CWASA, boosts the performance of all continuous space models. Particularly, $\mathrm{XCNN}$ benefits the most and becomes the best performing model in the corresponding category (c). This also reaffirms the fact that XCNN can learn discriminative representations. However, the simple addition of word-level representations limits its full potential, especially when the text fragment is large. It should also be noted that CWASA (XCNN) is not comparable to CL-KGA. While the former is trained on a limited parallel corpus and operates on 20k dimensional vocabulary, CL-KGA leverages on a large sophisticated multilingual resource such as BabelNet with more than 9M concepts.

The hybrid models in group (d) which combine knowledge graphs and continuous space models, produce the best results; even outperforming a strong model such as CL-KGA. This gives evidence that continuous space models are a good complement to discrete models. The high performance of these models suggests that latent semantic models provide powerful features for the candidate retrieval task of plagiarism detection.

\subsubsection{Experiment B: Cross-language plagiarism detection}

This second experiment aims at evaluating the detection of plagiarism cases at the fragment level. Different cross-language similarity estimation models are used in Algorithm 2 for fragment identification. The performance is evaluated on standard metrics for plagiarism detection task, such as precision, recall, granularity and 


\begin{tabular}{ll|llll|llll}
\hline & \multicolumn{3}{|c|}{ Spanish-English } & \multicolumn{4}{c}{ German-English } \\
Model & Plag & Prec & Rec & Gran & Plag & Prec & Rec & Gran \\
\hline (a) & CL-KGA & $\mathbf{0 . 6 2 0}$ & $\mathbf{0 . 6 9 6}$ & $\mathbf{0 . 5 5 8}$ & $\mathbf{1 . 0 0 0}$ & $\mathbf{0 . 5 2 0}$ & 0.601 & $\mathbf{0 . 4 6 0}$ & $\mathbf{1 . 0 0 4}$ \\
& CL-ASA & 0.517 & 0.690 & 0.448 & 1.071 & 0.406 & $\mathbf{0 . 6 0 4}$ & 0.344 & 1.113 \\
& CL-ESA & 0.471 & 0.535 & 0.448 & 1.048 & 0.269 & 0.402 & 0.230 & 1.125 \\
& CL-C3G & 0.373 & 0.563 & 0.324 & 1.148 & 0.115 & 0.316 & 0.080 & 1.166 \\
\hline (b) & S2Net & $\mathbf{0 . 5 1 4}$ & 0.734 & $\mathbf{0 . 4 4 0}$ & $\mathbf{1 . 0 9 8}$ & $\mathbf{0 . 3 7 9}$ & $\mathbf{0 . 6 6 9}$ & $\mathbf{0 . 3 0 4}$ & 1.148 \\
& XCNN & 0.386 & $\mathbf{0 . 7 3 8}$ & 0.310 & 1.189 & 0.270 & 0.664 & 0.196 & 1.174 \\
& CAE & 0.440 & 0.736 & 0.360 & 1.142 & 0.212 & 0.482 & 0.150 & $\mathbf{1 . 1 2 0}$ \\
\hline (c) & CWASA (XCNN) & $\mathbf{0 . 6 0 9}$ & 0.686 & $\mathbf{0 . 5 4 7}$ & $\mathbf{1 . 0 0 1}$ & $\mathbf{0 . 4 9 2}$ & $\mathbf{0 . 6 1 1}$ & $\mathbf{0 . 4 3 0}$ & $\mathbf{1 . 0 3 7}$ \\
& CWASA (S2Net) & 0.607 & $\mathbf{0 . 6 9 3}$ & 0.542 & 1.002 & 0.408 & 0.585 & 0.353 & 1.111 \\
& CWASA (CAE) & 0.354 & 0.546 & 0.296 & 1.121 & 0.237 & 0.478 & 0.176 & 1.122 \\
\hline (d) & KBSim (XCNN) & $\mathbf{0 . 6 4 4}{ }^{\dagger}$ & $\mathbf{0 . 7 6 5}$ & 0.556 & $\mathbf{1 . 0 0 0}$ & $\mathbf{0 . 5 6 1} \mathbf{1}^{\dagger}$ & $\mathbf{0 . 7 2 3}$ & 0.463 & 1.010 \\
& KBSim (S2Net) & 0.623 & 0.701 & $\mathbf{0 . 5 6 0}$ & $\mathbf{1 . 0 0 0}$ & 0.536 & 0.614 & $\mathbf{0 . 4 7 7}$ & $\mathbf{1 . 0 0 2}$ \\
& KBSim (CAE) & 0.622 & 0.704 & 0.557 & $\mathbf{1 . 0 0 0}$ & 0.521 & 0.592 & 0.468 & 1.004 \\
\hline
\end{tabular}

Table 6.3: ES-EN and DE-EN performance analysis in terms of plagdet (Plag), precision (Prec), recall (Rec) and granularity (Gran). The best results within each category are highlighted in bold-face and $\dagger$ represents statistical significance, as measured by a paired t-test $(p$-value $<0.05)$.

plagdet. These metrics were already described in Section 6.2.3.

The overall results are presented in Table 6.3. Similar to Experiment A, in general, performances over the DE-EN task are lower than the performances over the ES-EN task. Among the vector space models, grouped in category (a), CL-KGA produced the best results.

The continuous models grouped in category (b) interestingly exhibit very high precision. S2Net is the best among them as evidenced by plagdet, which combines precision, recall and granularity. As discussed before, the CWASA composition method in group (c) enhances the performance, especially for XCNN, making CWASA (XCNN) comparable to CL-KGA. Interestingly, CWASA (CAE) is worse than CAE, which suggests that CWASA is best suitable for models like XCNN that inherently produce word embeddings.

Finally, the hybrid models grouped in category $(d)$ produce the best results. Specifically, KBSim (XCNN) performs the best among all models for both language 
pairs. This observation (similar to the corresponding one in Experiment A) confirms that knowledge graphs and continuous space models capture different aspects of text and complement each other.

\subsection{Cross-language ad-hoc retrieval}

Cross-language ad-hoc retrieval addresses the situation where a system is presented with an information need in form of a few keywords. The system has to produce a ranked list of documents that are relevant to the provided information need. The CAE and XCNN models are evaluated on the standard ad-hoc retrieval task in the cross-language setting. Current search engines do not employ CLIR systems for web search because of several user-experience aspects such as presentation of results and query formulation. The most suitable use-cases are the following:

1. A bilingual user issuing a query in one language and assessing the results in a different language, where the relevant information is only available in the latter language.

2. A mono-lingual user issuing a query in one language and assessing results in the same language with the help of automatic translation systems, where the relevant information is only available in a different language.

An example of these scenarios are: a Spaniard with a limited knowledge of Eng-

lish, who visits U.K. and formulates a query in Spanish; or a Briton (who only knows English) who visits Spain and formulates a query in English. In case the user is not acquainted with the language of the retrieved documents, an automatic machine translation system can be used to present results in the language of user preference.

\subsubsection{Problem statement}

Let $\mathcal{D}$ denote a collection of documents in language $\mathcal{L}_{1}$ and information need is expressed by query $q$ in language $\mathcal{L}_{2}$. The task is to generate a ranked list $(\mathcal{R})$ of 
documents from $\mathcal{D}$ in decreasing order of relevance.

\subsubsection{Methods}

There are two main approaches to cross-language information retrieval: (i) machinetranslation, and (ii) cross-language latent semantic projections. In machine translation based approaches, an MT system from $\mathcal{L}_{2} \rightarrow \mathcal{L}_{1}$ is used to represent query $q$ in $\mathcal{L}_{1}$, and then, mono-lingual IR is carried out. In latent semantic projection based approaches, a cross-language projection function is used to represent both $\mathcal{D} \in \mathcal{L}_{1}$ and $q \in \mathcal{L}_{2}$ in a low-dimensional abstract, where semantic similarity is estimated.

\subsubsection{Datasets and experiments}

Our CLIR experimental evaluation is carried out on the FIRE 2011-12 En-Hi CLIR track corpu: $[1]$ It contains 100 English queries (topics), 331,599 news articles in Hindi and their corresponding relevance judgments (qrels). The corpus contains news articles that cover different domains including entertainment, politics, business, popular culture etc. The topics are formulated by browsing the corpus and refined further based on initial retrieval results to ensure the minimum number of relevant documents per query. This is to make a balance between easy, medium and hard queries. The collection contains binary relevance judgments generated through a pool of submitted runs (Palchowdhury et al., 2011). The retrieval results are evaluated by the standard IR metrics, more specifically, we used mean reciprocal rank (MRR), mean average-precision (MAP) and normalised discounted cumulative gain (nDCG) (Järvelin and Kekäläinen, 2002). MRR is described in Eq. 6.25 and nDCG is described in Sec. 2.1.3,

$$
\operatorname{MRR}=\frac{1}{|Q|} \sum_{i=1}^{|Q|} \frac{1}{\operatorname{rank}_{i}}
$$

\footnotetext{
$1 1 \longdiv { \text { http://www.isical.ac.in/ fire/ } }$
} 
where $Q$ is the query-set and $\operatorname{rank}_{i}$ is the rank of the first relevant document for query $i$.

For training the latent semantic models and the MT system, the En-Hi parallel corpus available from WMT 2014 (Bojar et al., 2014b) was used. In total, 100k parallel sentences from the corpus were used, which at least contained 3 terms from the selected vocabulary. The selected vocabulary consisted of $20 \mathrm{k}(10+10)$ most frequent terms, which were selected after removing stopwords. A stemmer is used applied to represent text.

\subsubsection{Results and analysis}

Here, we present the results and analysis of XCNN for the task of cross-language adhoc retrieval (Gupta et al., 2016a). In order to step-by-step analyse XCNN learning, the monolingual pre-initialisation is evaluated first, because that makes the basis for the cross-language extension. We compare its performance with a standard BM25 baseline to ensure reliability of the model, referred as mono-XCNN. At this stage mono-XCNN is not expected to outperform the vector space model because the final objective of the model is to learn cross-language associations. Naturally, the FIRE collection does not provide click-through data. In absence of click-through data, we use pseudo-relevance data as positive samples for training data. Concretely, the sentence with the highest BM25 score w.r.t. the input sentence is chosen as positive sample for it. The document collection is in Hindi, hence we report results with Hindi queries in monolingual setting. The code for the XCNN model is publicly available at: https://github.com/parthg/jDNN

The results using Hindi queries are presented in Table 6.4. In the table, BM25 and mono-XCNN are evaluated using a limited vocabulary of size 10k. Interestingly, for the top rank-position related metrics like nDCG@1 and MRR, mono-XCNN performs better than BM25. For other metrics which involve lower rank positions, the performance of mono-XCNN is sub-optimal to the VSM approach. This is not sur-

\footnotetext{
${ }^{12}$ ACL 2014 ninth workshop on statistical machine translation http://www . statmt . org/wmt14/.
} 
prising because, in our experimental setting, pseudo relevance data comes from the BM25 scores and mono-XCNN is trained to optimise it; hence it is ought to be upper-bounded by the BM25 scores for lower dimensions. We also expect this gain to be much higher if clickthrough data is used instead of pseudo relevance data. This result is also consistent with other works, in which it is shown that using only latent models in monolingual setting might hurt the ranking performance, especially for the case of very low dimensional latent space (Manning and Schütze, 1999; Gao et al. 2011).

\begin{tabular}{|l|c|c|c|c|c|}
\hline Method & nDCG@1 & nDCG@5 & nDCG@10 & MAP & MRR \\
\hline BM25 & 0.2800 & 0.2814 & 0.2758 & 0.0957 & 0.3851 \\
mono-XCNN & 0.3000 & 0.2472 & 0.2233 & 0.0794 & 0.4173 \\
\hline
\end{tabular}

Table 6.4: Results for the monolingual ad-hoc retrieval task measured in nDCG, MAP and MRR.

For the cross-language setting, the retrieval performance is presented in Table6.5. It can be noticed that XCNN outperforms all the models. The difference between $\mathrm{XCNN}$ results and those from the rest of the models is statistically significant, as measured by a paired t-test $(p$-value $<0.05)$. The linear projection based techniques: CL-LSI, OPCA and S2Net, perform close to each other with non-significant differences. Also, as seen from the table, the overall results for this task are low. This is mainly because of two reasons: $(i)$ the selected vocabulary does not cover all the query and document terms, resulting in many out-of-vocabulary (OOV) terms in both the queries and the articles, and (ii) the parallel training data is not large enough $\mathrm{h}^{13}$ and contains a mixture of domains different from the one of the FIRE corpus. However, this situation affects equally all the models, which provides a fair ground for comparison.

In order to alleviate this problem, new experiments are conducted by considering only those queries for which at least $80 \%$ of terms are present in the vocabulary ${ }^{14}$.

\footnotetext{
${ }^{13}$ Hindi is a resource-constrained language and the largest parallel corpus is a few hundred thousand sentences, while other resource rich languages have parallel data of a few millions sentences.

${ }^{14}$ In total, there are 80 such queries out of 100 .
} 
The results are presented in Table 6.6.

\begin{tabular}{|l|c|c|c|c|c|}
\hline Method & nDCG@1 & nDCG@5 & nDCG@10 & MAP & MRR \\
\hline CL-LSI & 0.1200 & 0.0544 & 0.0420 & 0.0062 & 0.1471 \\
OPCA & 0.1300 & 0.0806 & 0.0663 & 0.0254 & 0.1573 \\
S2Net & 0.1263 & 0.0823 & 0.0734 & 0.0278 & 0.1837 \\
CAE & 0.1588 & 0.1136 & 0.1057 & 0.0310 & 0.2136 \\
MT & 0.1800 & 0.1333 & 0.1273 & $\mathbf{0 . 0 4 1 8}$ & 0.2537 \\
XCNN & $\mathbf{0 . 2 2 0 0}^{\dagger}$ & $\mathbf{0 . 1 5 2 5}^{\dagger}$ & $\mathbf{0 . 1 3 1 2}^{\dagger}$ & 0.0386 & $\mathbf{0 . 3 1 2 8}^{\dagger}$ \\
\hline
\end{tabular}

Table 6.5: Results for the ad-hoc retrieval task measured in $\mathrm{nDCG}, \mathrm{MAP}$ and MRR for title topic field. The best results are highlighted in bold-face and $\dagger$ represents statistical significance, as measured by a paired t-test $(p$-value $<0.05)$.

\begin{tabular}{|l|c|c|c|c|c|}
\hline Method & nDCG@1 & nDCG@5 & nDCG@10 & MAP & MRR \\
\hline CL-LSI & 0.1463 & 0.0591 & 0.0416 & 0.0069 & 0.1639 \\
OPCA & 0.1524 & 0.0914 & 0.0762 & 0.0291 & 0.1790 \\
S2Net & 0.1603 & 0.1003 & 0.0826 & 0.0334 & 0.2103 \\
CAE & 0.1690 & 0.1129 & 0.1067 & 0.0354 & 0.2332 \\
MT & 0.1707 & 0.1278 & 0.1224 & 0.0411 & 0.2538 \\
XCNN & $\mathbf{0 . 2 6 8 3}^{\dagger}$ & $\mathbf{0 . 1 7 8 7 ^ { \dagger }}$ & $\mathbf{0 . 1 5 3 5}^{\dagger}$ & $\mathbf{0 . 0 4 5 9}^{\dagger}$ & $\mathbf{0 . 3 7 1 1}^{\dagger}$ \\
\hline
\end{tabular}

Table 6.6: Results for the ad-hoc retrieval task measured in nDCG, MAP and MRR for title topic field considering only those queries for which more than $80 \%$ query-terms appear in the vocabulary. The best results are highlighted in bold-face and $\dagger$ represents statistical significance, as measured by a paired t-test $(p$-value $<0.05)$.

It has been reported that S2Net parameters can be initialised randomly or from CL-LSI or OPCA projection matrices (Yih et al., 2011). Similarly, it is possible to initialise XCNN parameters easily with the parameters obtained through autoencoders. In this work, we initialised these models' param eters randomly. This is done because of two reasons: $(i)$ we are primarily interested in comparing XCNN with S2Net and we wanted to study the abilities of these models to learn semantically plausible representations without dependence on any external method, and $(i i)$ the complexity of computing the matrix factorization required by CL-LSI and OPCA scales quadratically with the vocabulary size, which makes such dependence com- 
putationally impractical for high dimensional applications such as ad-hoc retrieval. Interestingly, as seen in the tables, XCNN is also able to outperform the MT based method. This confirms that XCNN is able to capture useful cross-language semantic representations within a very low dimensional space.

\subsection{Cross-language parallel sentence retrieval}

With the advent of the web, cross-language information retrieval becomes important not only to satisfy the information need across languages but to mine resources across multiple languages, such as for example parallel or comparable documents. Such mined resources aid training machine translation systems (Munteanu and Marcu, 2005: Türe and Lin, 2012). In this sense, the aim of cross-language parallel sentence retrieval is to find parallel counterparts across different languages for a given sentence, or text fragment.

\subsubsection{Problem statement}

Let $\mathcal{S}$ denote a collection of sentences in language $\mathcal{L}_{1}$ and $q$, an input sentence in language $\mathcal{L}_{2}$. The task of parallel sentence retrieval is to find potential translations for input sentence $q$ in $\mathcal{S}$.

\subsubsection{Datasets and experiments}

The En-Hi parallel corpus available from WMT 2014 $4^{15}$ is used for training and evaluation. The parallel sentences come from various sources like news articles, commentaries, Wikipedia, TED talks etc. More details on the corpus is available in Bojar et al. (2014b). The corpus contains a total of $274 \mathrm{k}$ parallel sentences. The working vocabulary was extracted by removing stopwords, applying stemming and keeping the most frequent 20k words (10k for each language). Finally, 122k parallel sentences,

\footnotetext{
${ }^{15}$ ACL 2014 ninth workshop on statistical machine translation http://www . statmt .org/wmt14/.
} 
which at least contained 3 terms from the vocabulary, were used for training (100k) and evaluation (the remaining 22k) ${ }^{16}$ For a fair comparison, all the models were trained and evaluated over the same training and evaluation partitions and with the same vocabulary.

\subsubsection{Results and analysis}

The results for the sentence retrieval task are presented in Table 6.7. The retrieval quality for each test sentence is assessed by considering its parallel counterpart's reciprocal rank in the rank-list. For this, we have used the MRR as evaluation metric.

In general, the models including a noise-contrastive component outperform the ones without it; e.g. OPCA vs. CL-LSI, and $\{\mathrm{XCNN}, \mathrm{S} 2 \mathrm{Net}\}$ vs. CAE. It suggests that having such component lead to better representation learning. It should also be noted that models such as S2Net and XCNN, which directly optimise the evaluation metric (cosine similarity) outperform the rest of latent space models such as CL-LSI, OPCA and CAE. It can be noticed from Table 6.7, that the proposed method clearly outperforms the other methods. Moreover, the observed difference is statistically significant ( $p$-value less than 0.01 ) according to the paired t-test. It should also be noted that each non-linear model outperforms its corresponding linear counterparts; e.g. CAE vs. $\{\mathrm{CL}-\mathrm{LSI}, \mathrm{OPCA}\}$, and XCNN vs. S2Net.

\subsection{Source context for machine translation}

In this section, we present the problem of lexical selection in machine translation. Such problem is handled with source context features. First, we describe the source context features and then show how the continuous space model CAE (c.f. Sec. 4.1) is used to provide such feature (Gupta et al., 2016b).

Source context is usually very relevant when translating texts. However, standard

\footnotetext{
${ }^{16}$ Many sentences were just one word being named entities extracted from Wikipedia page titles.
} 


\begin{tabular}{l|c}
\hline Method & MRR \\
\hline CL-LSI & 0.2620 \\
OPCA & 0.4349 \\
CAE & 0.4789 \\
S2Net & 0.4638 \\
MT & 0.4876 \\
XCNN & $\mathbf{0 . 5 3 2 8}^{\dagger}$ \\
\hline
\end{tabular}

Table 6.7: Results for the parallel sentence retrieval task measured in MRR. The best results are highlighted in bold-face and $\dagger$ represents statistical significance, as measured by a paired t-test $(p$-value $<0.01)$.

phrase-based statistical machine translation systems use a source context that is limited to the span of the used translation units. The source context information becomes specially necessary when using the same translation system for translating texts from different domains. Also, the source-context information is important for dealing with both polysemy and morphology, in which the source language has words with the same form (spelling) that can be translated into different forms in the target language. In this task, our CAE model is used to provide source context information to a standard phrase-base machine translation system. The proposed feature is explained in Section 6.5.1 and the effectiveness of the method is evaluated on a machine translation task.

\subsubsection{Source-context feature}

The main idea behind the proposed source context feature is an extended concept of translation unit or phrase $(p)$, which is defined by a unit of three elements: phrasesource $(p s)$, phrase-target $(p t)$ and source-sentence $(s s)$.

$$
p=\{p s|||p t||| s s\}
$$

From this definition, identical source-target phrase pairs that have been extracted from different training sentence pairs are regarded as different translation units. 
According to this, the relatedness of contexts can be considered as an additional feature function $(s c f)$ for each phrase and input sentence.

The source-context feature function consists of a similarity measurement between the input sentence to be translated and the source context component of the available translation units as illustrated in Fig. 6.2.

S1: it was difficult to park the car

T1:es difícil aparcar el coche

S2: the flowers are beautiful in the park

T2:las flores son hermosas en el parque

Input: children are playing in the park

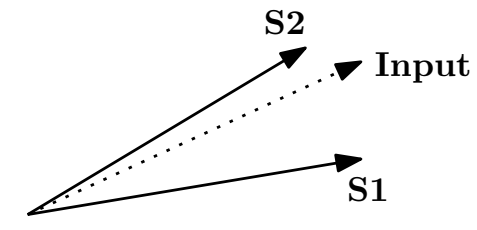

park : parque $\checkmark$

park : aparcar $X$

Figure 6.2: Illustration of the proposed similarity feature to help choosing translation units.

This $s c f$ is included for each phrase within the translation table in addition to the standard feature functions: conditional $(c p)$ and posterior $(p p)$ probability, lexical weights $(l 1, l 2)$ and phrase bonus $(p b)$. This schema was originally proposed by Banchs and Costa-jussà (2011). In our proposed implementation, the calculation of $s c f$ is carried with our model CAE. The work-flow of our proposed implementation is depicted in Fig. 6.3.

\subsubsection{Datasets and experiments}

We used an English-to-Spanish parallel corpus extracted from the Bible. It constitutes an excellent corpus for experimenting with and testing the proposed methodology as it provides a rich variety of contexts. The corpus contains around 30,000 


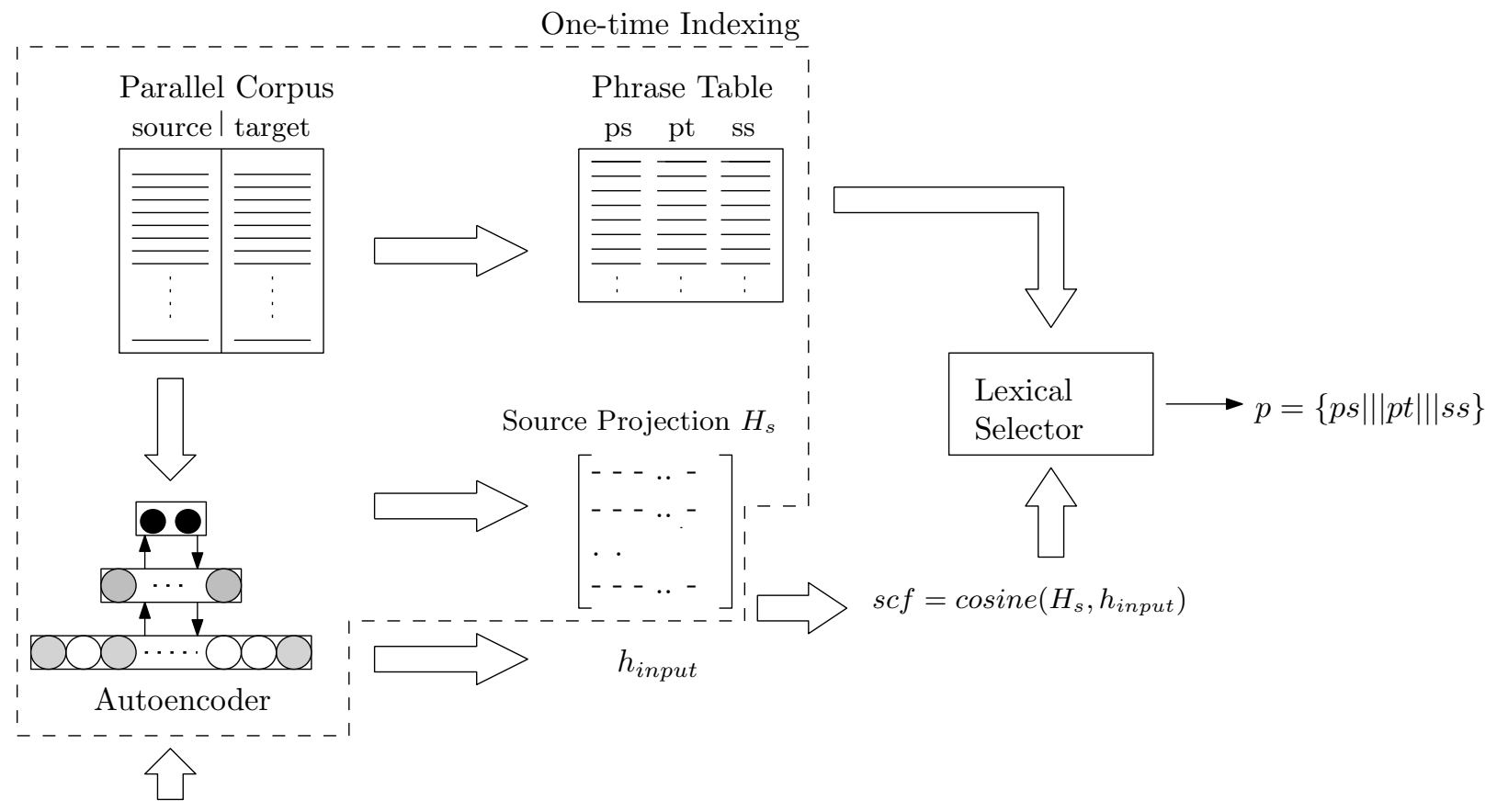

Input Source Sentence

Figure 6.3: Workflow of the system.

sentences for training with around 800,000 words, and 500 sentences for each development and test sets. Additionally, for testing the system over a large size dataset, we used the English-to-Hindi corpus available from WMT 2014 (Bojar et al., 2014b). In this case, the dataset comprises 300,000 sentences, with 3,500,000 words, 429 sentences for development and 500 sentences for test. We evaluated the effect of incorporating $s c f$ by using different menthods and estimated the quality of machine translation in terms of bilingual evaluation understudy (BLEU) metric. BLEU calculates a modified version of precision in n-gram space to measure similarity of the generated translations with that of the reference translations. It is an average n-gram precision score with some smoothing factors and length penaltiesusing geometric averages (Papineni et al. 2002). 


\subsubsection{Results and analysis}

Tables 6.8 shows the improvements in terms of BLEU of adding the proposed source context feature to the baseline system for English-to-Spanish (En2Es) and English-toHindi (En2Hi), respectively. As shown in the tables, the proposed method performs significantly better than the baseline for both translation tasks. (Durrani et al., 2014) depicts the best published results for En2Hi translation task on WMT dataset (Bojar et al., 2014a).

\begin{tabular}{|l|c|c|c|c|}
\cline { 2 - 5 } \multicolumn{1}{c|}{} & \multicolumn{2}{c|}{ En2Es } & \multicolumn{2}{c|}{ En2Hi } \\
\cline { 2 - 5 } \multicolumn{1}{c|}{} & Dev & Test & Dev & Test \\
\hline baseline & 36.81 & 37.46 & 9.42 & 14.99 \\
(Durrani et al., 2014$)$ & NA & NA & NA & 12.83 \\
+CL-LSI & $37.20^{*}$ & $37.84^{*}$ & $9.83^{*}$ & $15.12^{*}$ \\
+CAE & $\mathbf{3 7 . 2 8}^{* \dagger}$ & $\mathbf{3 8 . 1 9}^{* \dagger}$ & $\mathbf{1 0 . 4 0}^{* \dagger}$ & $\mathbf{1 5 . 4 3}^{* \dagger}$ \\
\hline
\end{tabular}

Table 6.8: BLEU scores for En2Es and En2Hi translation tasks. ${ }^{*}$ and ${ }^{\dagger}$ depicts statistical significance $(p$-value $<0.05)$ wrt Baseline and LSA respectively.

It can be noticed that the results from En2Es and En2Hi are consistently improved. Both, Hindi and Spanish, have a higher vocabulary variation compared to English, with richer morphology. The improvements in BLEU suggests that the continuous space representation helps finding the adequate contextual similarities among the training and test sentences. BLEU scores show improvement over all tasks and translation directions. Further analysis of the translation outputs, using ASIYA ${ }^{17}$, revealed some examples of how the translation is improved in terms of lexical selection. The examples are shown in Table 6.9.

Table 6.10 presents in further detail the feature values involved in the phrase selections of the examples in Table 6.9. From it, the role of $s c f$ in lexical selection can be clearly appreciated, which reflects the main reasons for improvement. It can be noticed from the Table 6.10 that the most probable sense of bands in our considered

\footnotetext{
${ }^{17}$ http://www.asiya.lsi.upc.edu
} 


\begin{tabular}{|l|l|}
\hline System & Translation \\
\hline Source & but he brake the bands \\
CL-LSI & pero él rompió las tropas \\
CAE & pero él rompió las cuerdas \\
Reference & pero él rompió las ataduras \\
\hline Source & soft cry from the depth \\
CL-LSI & गहराइयों से मूलायम रोने लगते \\
CAE & गहराइयों से मूलायम चीख \\
Reference & गहराइयों से कोमल चीख \\
\hline
\end{tabular}

Table 6.9: Manual analysis of translation outputs. Adding the source context similarity feature allows for a more adequate lexical selection.

\begin{tabular}{|c|c|c|c|}
\cline { 2 - 4 } \multicolumn{1}{c|}{} & $\boldsymbol{c p}$ & $\boldsymbol{p p}$ & $\boldsymbol{s c f}$ \\
\hline bands|||tropas & 0.31 & 0.17 & 0.01 \\
bands || cuerdas & 0.06 & 0.07 & 0.23 \\
\hline cry || रोना & 0.23 & 0.06 & 0.85 \\
cry || चीख & 0.15 & 0.04 & 0.90 \\
\hline
\end{tabular}

Table 6.10: Probability values (conditional and posterior as standard features in a phrasebased system) for the word bands and two Spanish translations; and the word cry and two Hindi translations.

dataset is tropas, which literally means "troups". However, for the specific context under consideration "troups" does not provide a correct translation option, which is clearly discriminated by $s c f$ as seen in Table 6.10. Therefore, given the entire input sentence (in): And he was kept bound with chains and in fetters; and he brake the bands, the method is be able to infer the correct sense for the word bands (i.e., in this case cuerdas, which literally means "ropes", a synonym of the reference ataduras, which literally means "tying with ropes") by considering its similarity to the training sentences: (s1) and the lord sent against him bands of the chaldees, and bands of the syrians and (s2) they shall put bands upon thee, and shall bind thee with them. In this case, $\omega(s 2, i n)>\omega(s 1, i n)$ as seen in Table 6.10. Similarly, in the Hindi example, the most frequent sense of word cry is रोना, which literally means "to cry" while the 
example in Table 6.9 refers to the sense of cry as चीख, which means to scream. Our method could identify the context and hence the $s c f$ (cry $\|$ चीख) $>s c f(\operatorname{cry} \| \mid$ रोना).

\subsubsection{Scalability}

There are two components of this method: $(i)$ incorporation of source-context features during the tuning phase of MT and projection of training sentences in the latent space; and $(i i)$ similarity estimation of the input sentence with the training sentences in the latent space. The former step is computationally expensive but it being one-time and offline, it is not a big concern. On the other hand, the similarity estimation is online. It can be efficiently implemented by using a multi-core CPU or GPU as it is essentially a matrix multiplication. 

Chapter

\section{Bottleneck dimensionality for autoencoders}

Lately, dimensionality reduction techniques based on deep learning have become very popular, especially deep autoencoders (Hinton and Salakhutdinov, 2006). Deep autoencoders can extract highly useful and compact features from the structural information of the data. Deep autoencoders have proven to be very effective in learning reduced space representations of the data for similarity estimation (Hinton and Salakhutdinov, 2006; Salakhutdinov and Hinton, 2009a). Deep learning is inspired by biological studies, which state the brain has a deep architecture. Despite their high suitability to the task, deep learning did not find much audience until Hinton and Salakhutdinov (2006) proposed a pre-training method to initialise the network parameters in a good region for finding optimal solutions.

Although deep learning techniques are in vogue, there still exist some important open questions. In most of the studies involving the use of these techniques for dimensionality reduction, the qualitative analysis of the obtained projections is seldomly presented. This makes the assessment of the reliability of learning very difficult. 
Typically, the reliability of the autoencoder is estimated based on its reconstruction capability.

The first objective of this chapter is to introduce a novel framework for evaluating the quality of the low-dimensional embeddings produced by a deep autoencoder based on the merits of the application under consideration. Concretely, the framework is comprised of two metrics, structure preservation index (SPI) and similarity accumulation index (SAI), which capture different aspects of the autoencoder's reconstruction capabilities, including the structural distortion and similarities among the reconstructed vectors (Gupta et al., 2016c). In this way, the framework gives better insight of the autoencoder performance allowing for conducting better error analysis and evaluation. The adequacy of the bottleneck dimension, referred to as critical bottleneck dimensionality here, is rarely addressed in the literature. These metrics also provide a better means for estimating the adequate size of critical bottleneck dimensions.

The second objective is to conduct a comparative evaluation about the dimensionality reduction capabilities of deep autoencoders across different languages. With this empirical evaluation, we aim at shedding some light regarding the adequacy of using the same number of dimensions when computing low-dimensional embeddings for different languages, which is a common practice in the field.

The dimensionality reduction experiments presented in this chapter are carried out on text at sentence level. The suitability of two types of deep autoencoders (c.f. Section 3.4) is assessed: (i) deep autoencoder with stochastic RBM (bDA); and (ii) deep autoencoder with multinomial RBM (rsDA). We report some interesting findings at the architectural level with regards to the specific problem of modelling text at the sentence level.

The chapter is structured as follows. Section 7.1 gives details about the analysis framework of the autoencoder learning, experiments and results. The discussion on critical bottleneck dimensionality and an automatic way to estimate it is given in Section 7.2. In Section 7.3, we attempt to see whether any correlation exists between 
the critical bottleneck dimensionality for a particular language and its perplexity.

\subsection{Qualitative analysis and metrics}

In this section, the metrics used for comparing the two considered autoencoder models (bDA and rsDA) are described. Subsequently, we present the comparative analysis of the two models.

The quality of the projections and the sufficiency of a given dimensionality $m$ are measured by the autoencoder's reconstruction ability. Unfortunately, the mean squared error between the input $x$ and its reconstruction $\hat{x}$, referred to as reconstruction error 3.12 , is a poor measure of the quality of the obtained projections. It neither gives any details about the quality of the reconstructions in terms of text data representation nor the degree to which the structure of the data is preserved in the reconstruction space. Moreover, it is difficult to justify the adequacy of bottleneck dimensionality $m$ by simply using the reconstruction error.

In the literature, when autoencoders are used for dimensionality reduction of text data, the quality is measured in terms of the accuracy of the end-task, which may be text categorisation (Hinton and Salakhutdinov, 2006), information retrieval (Salakhutdinov and Hinton, 2009a), topic modeling (Salakhutdinov and Hinton, 2009b), term modeling across scripts (Gupta et al., 2014) or sentiment prediction (Socher et al., 2011). A shortcoming of this approach is that there is no way to estimate the full potential, or upper bound, of the algorithm performance. On the other hand, in the case of poor results, it becomes difficult to determine whether the training was proper or not.

\subsubsection{Metrics}

In this chapter we introduce two new metrics, which are intended to capture different aspects of the autoencoder's reconstruction capability: (i) structure preservation index (SPI), and (ii) similarity accumulation index (SAI). These two metrics focus 
their attention on the structural distortion and semantic similarity of the reconstructed vectors with respect to the original ones. These two metrics, along with the reconstruction error, allow for a much better assessment of confidence regarding the quality of the network training process and its performance.

(i) Structure preservation index Consider the input data as $\mathbf{X}$ where each row $X_{i}$ corresponds to the vector space representation of the $i^{\text {th }}$ document and $\hat{\mathbf{X}}$ is its corresponding reconstruction. $\mathbf{X}$ and $\hat{\mathbf{X}}$ are $p \times n$ matrices where $p$ is the total number of documents and $n$ is the vocabulary size. Compute the $p \times p$ matrix $D$ for $\mathbf{X}$ such that $D_{i j}$ is the cosine similarity score between $i^{t h}$ and $j^{\text {th }}$ rows of $\mathbf{X}$. Similarly calculate the $p \times p$ matrix $\hat{D}$ for $\hat{\mathbf{X}}$. $D$ and $\hat{D}$ can be seen as similarity matrices of the original data and its reconstruction, respectively, where $D_{i j}=\hat{D}_{i j}=1, \forall i=j$. The SPI is calculated as follows:

$$
\mathrm{SPI}=\frac{1}{p^{2}} \sum_{i j}\left\|D_{i j}-\hat{D}_{i j}\right\|^{2}
$$

Notice that according to this definition, SPI captures the structural distortion incurred by the encoding and decoding processes. Ideally, SPI should be zero.

(ii) Similarity accumulation index Different from SPI, which assesses structural distortion, SAI attempts to capture the quality of the reconstructed vectors by measuring the cosine similarity between each original vector and its reconstructed version. Indeed, this metric assesses how well aligned are the vector-dimensions in the reconstruction with respect to the original vectors.

SAI is computed by the normalised accumulation of cosine similarities between each input document and its reconstruction. Ideally, SAI should be one:

$$
\mathrm{SAI}=\frac{1}{p} \sum_{i=1}^{p} \operatorname{cosine}\left(x_{i}, \hat{x}_{i}\right)
$$




\subsubsection{Comparative evaluation of models}

In this section, we present an experimental comparison between the bDA and the rsDA models when conducting dimensionality reduction of texts at the sentence level, where data sparseness plays a more critical role than in the case of full documents. This study aims at exploring the use of autoencoder techniques for sentence-centered applications, such as machine translation, text summarization and automatic dialogue response.

For the experiments presented in this chapter, we use the Bible dataset, which contains 25122 training and 995 test sentences. All sentences were processed by a term-pipeline of stopword-removal and stemming to obtain the vocabulary which is referred as Vocab $\mathbf{V}_{1}$. In addition, we also kept only those terms which were nonnumeric, at least 3-characters long and appeared in at least 5 training sentences. We refer to this filtered vocabulary as $\mathbf{V o c a b}_{2}$. For the English partition of the dataset, Vocab $_{1}$ and Vocab ${ }_{2}$ sizes are 8279 and 3100, respectively.

We train the autoencoder models for English as described in Section 4.1 .3 and evaluated the the quality of the reconstructions in terms of the reconstruction error (RC) and the two proposed metrics SPI and SAI. The results are presented in Table 7.1 .

\begin{tabular}{|l|ccc|}
\hline Model & RC & SPI & SAI \\
\hline rsDA $(p t)$ & 0.1192 & 0.7258 & 0.2132 \\
rsDA $(b p)$ & 0.0834 & 0.0049 & 0.5768 \\
\hline bDA $(p t)$ & 8.0012 & 0.0712 & 0.3528 \\
bDA $(b p)$ & 5.4829 & 0.0035 & 0.6667 \\
\hline
\end{tabular}

Table 7.1: The performance of bDA and rsDA in terms of different metrics. RC, SPI and SAI denote reconstruction error, structure preservation index and similarity accumulation index while $p t$ and $b p$ denote if the model is only pre-trained or fine-tuned after pre-training, respectively. 


\subsubsection{Analysis and discussion}

When constructing low-dimensional embeddings for representing a dataset, it is important to understand the amount of distortion incurred by the network on the structure of the data during the process of encoding and decoding. During the training phase, the network uses the reconstruction error to update its parameters but the reconstruction error does not give much insight about the quality of the resulting low-dimensional representations. Another limitation of the reconstruction error is that it is not bounded and then not comparable across different models e.g. bDA vs. rsDA (see Table 7.1).

The two proposed metrics, SPI and SAI are both bounded and then comparable across the models. SPI measures how the similarity structure of sentences among each other is preserved in the reconstruction space, which in turn gives a measure of trustworthiness of the network for similarity estimation. Although both models show similar performance in terms of SPI after backpropagation, bDA is $28.57 \%$ better than rsDA according to SPI.

It is also important to assess the similarity between each input vector and its corresponding reconstruction. This is captured by SAI. According to SAI, bDA is 15.59\% better than rsDA. This is better illustrated in Fig. 7.1, where the histograms of cosine similarity between the original and reconstructed samples are presented for both bDA and rsDA. As it can be noticed in the figure, in the case of rsDA, for more than half of the test samples, the cosine similarity with their reconstruction is $\leq$ 0.6. Although rsDA has been reported in the literature to better perform at the document level, our results demonstrate that bDA is a more suitable model to be used when using autoencoder representations at the sentence level. This can be explained by the fact that rsDA uses multinomial sampling to model the word-counts, which happens not to be suitable at the sentence level for three reasons: $(i)$ most of the terms typically appear only once in a sentence, $(i i)$ sampling the distribution of terms by text length $D$ is less reliable when $D$ is small (e.g. sentence vs. full document, and (iii) the gradients at the output layer (softmax) in rsDA are very 


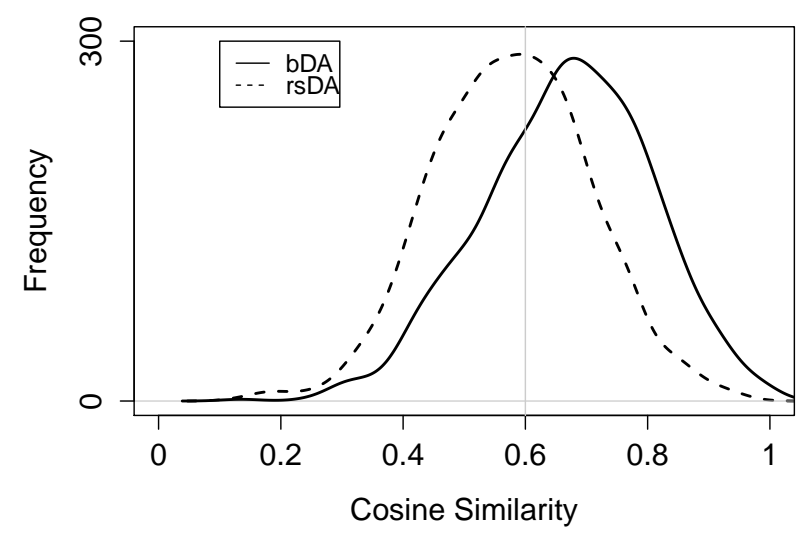

Figure 7.1: Histogram of cosine similarity between test samples and their reconstructions for bDA and rsDA.

small as they are calculated over a probability distribution.

Finally, as argued by Erhan et al. (2010), pre-training helps to initialise the network parameters in a good region that is close to the optimal solution. It can clearly be noticed that pre-training is necessary but itself is not enough to put aside backpropagation.

\subsection{Critical bottleneck dimensionality}

In this section we explore the implications of the size of bottleneck layer in the reconstruction quality of a given autoencoder. Later, we extend the analysis to a multilingual scenario and describe an automatic method to estimate the critical bottleneck dimensionality for different languages.

The central hidden layer of an unrolled autoencoder is commonly referred to as the bottleneck layer. The reconstruction ability of the autoencoder is highly related to the size of the bottleneck layer, in the sense that the smaller the size of the bottleneck layer, the higher the loss of information. 
The reduction step of autoencoders is also called hashing, and because similar sentences in the projected space are near to each other, this technique is also referred to as semantic hashing. It is important to choose a proper size of the bottleneck layer because of two reasons: (i) large dimensionalities may lead to redundant dimensions and limited abstraction capabilities, and (ii) small dimensionalities might lead to an excess of information loss.

The best compromise between information loss and abstraction power in terms of the bottleneck dimension, which we refer to as critical bottleneck dimensionality here, is rarely addressed in the literature. In this section, we present an analysis on the effects of choosing different sizes for the bottleneck layer, as well as we provide an empirical method to choose the critical bottleneck dimensionality.

\subsubsection{Metric selection}

In our exploratory experiments the bottleneck layer of the autoencoder is squeezed to identify whether there is a dimensionality region at which the reconstruction error, SPI and SAI metrics exhibit a clear change in behaviour. Typically, this region is referred to as the "elbow region". The autoencoder is trained by varying down the size of the bottleneck layer from 100 to 10 with step-sizes of 10. Fig. 7.2 shows the values of reconstruction error, SPI and SAI for the different considered sizes of bottleneck layer.

As it becomes evident from the figure, SPI is the metric exhibiting the clearest "elbow region" pattern. Indeed, it can be noticed that both the reconstruction error and SAI show a quasi-linear behaviour with almost constant slope, while SPI clearly captures that below $m=40$, the network starts losing information about the structure of the data at a faster rate. This result shows that care must be taken to select a proper bottleneck dimensionality, as well as it is important not to choose the bottleneck dimensionality below the point where SPI changes its behaviour. 

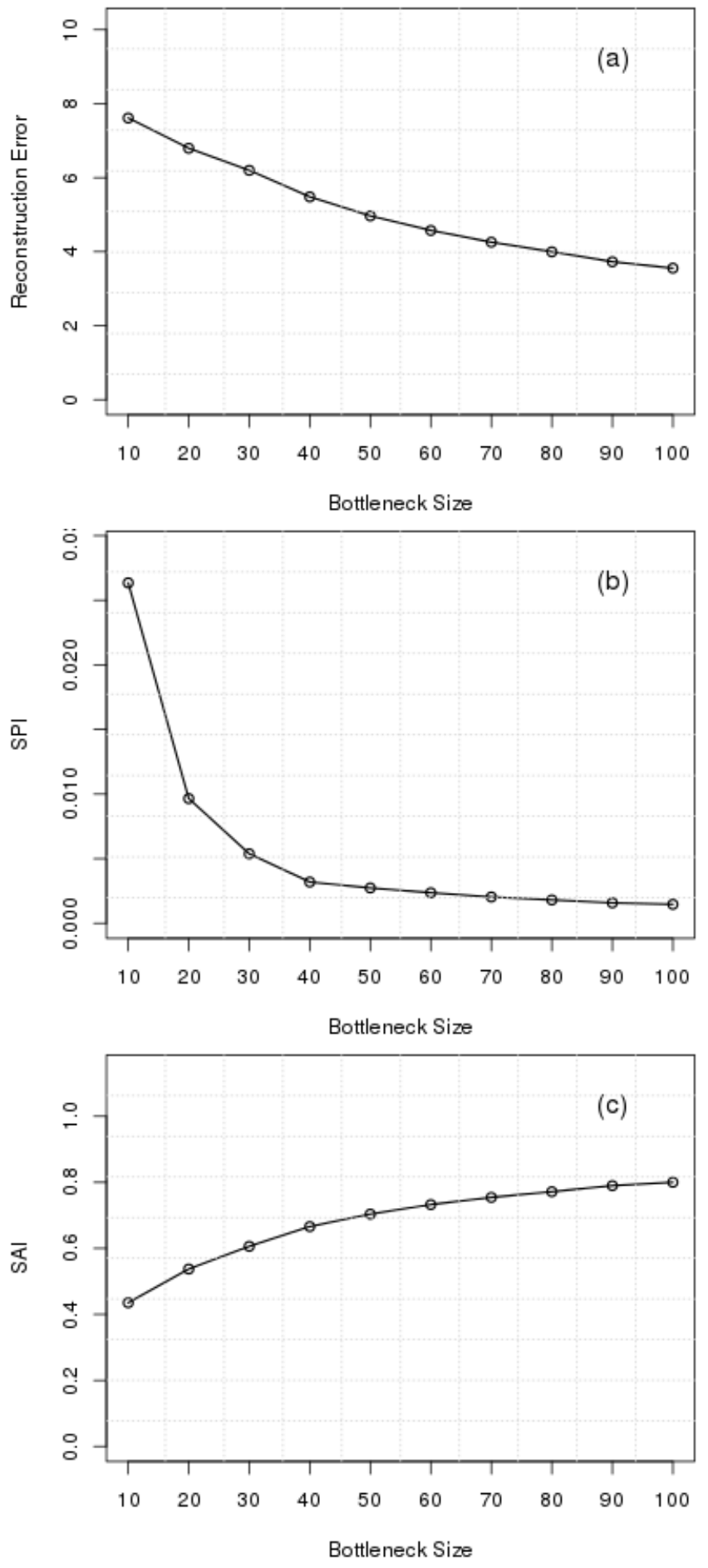

Figure 7.2: Reconstruction error, SPI and SAI metrics when varying the bottleneck layer size from 100 to 10 are shown in (a), (b) and (c), respectively. 


\subsubsection{Multilingual analysis}

Typically, in cross- and multi-language dimensionality reduction techniques, the documents are projected to a common abstract space for which the dimensionality is selected regardless the involved languages. Based on the analysis presented in the previous section addressing the importance of properly identifying the critical bottleneck region, we want to further explore the following question: does a common dimension suit all the languages?

To understand this phenomenon, we conduct a comparative study by considering different-language partitions of the same English dataset described in Section 7.1.2. Due to language pre-processing capabilities, we restricted our study to 5 different languages: English (Indo-European/Germanic), Spanish (Indo-European/Italic), Russian (Indo-European/Balto-Slavic), Turkish (Turkic) and Arabic (Afro-Asiatic). We repeated the experiment described in Section 7.2 .1 for all these 5 languages. The vocabulary sizes of these languages are depicted in Table 7.2 . The fundamental idea

\begin{tabular}{lcc}
\hline Language & $\mid$ Vocab $_{1} \mid$ & $\left|\mathbf{V o c a b}_{2}\right|$ \\
\hline English (en) & 8279 & 3100 \\
Spanish (es) & 9398 & 3581 \\
Russian (ru) & 18285 & 4504 \\
Turkish (tk) & 17087 & 4502 \\
Arabic (ar) & 18703 & 3012 \\
\hline
\end{tabular}

Table 7.2: Vocabulary sizes of the Bible dataset.

behind this experiment is to see whether the same information in different languages can be represented on a reduced dimensionality space of the same size. We anticipated that the critical bottleneck dimensionality of each language can be affected by different parameters like: its vocabulary size, its syntactic structure and its semantic complexity.

To identify the critical bottleneck dimensionality for each language, the percentage difference between the slopes connecting consecutive bottleneck sizes in the SPI curve is calculated. This captures the point in the "elbow region" of the SPI curve 
with steepest slope. Consider three points in the SPI plot: $a_{1}, a_{2}$ and $a_{3}$. Let $s_{1}^{2}$ and $s_{2}^{3}$ be the slopes of the lines connecting $a_{1}-a_{2}$ and $a_{2}-a_{3}$, respectively. Then the percentage difference between $s_{1}^{2}$ and $s_{2}^{3}$ gives the steepness of the curve at point $a_{2}$. We calculate this value for every point in the range in order to identify the critical dimensionality, as the point in which the percentage difference is the largest. This method enables us to automatically find the adequate bottleneck dimension for a particular language. The algorithmic implementation of this method is described in Algorithm. 3 .

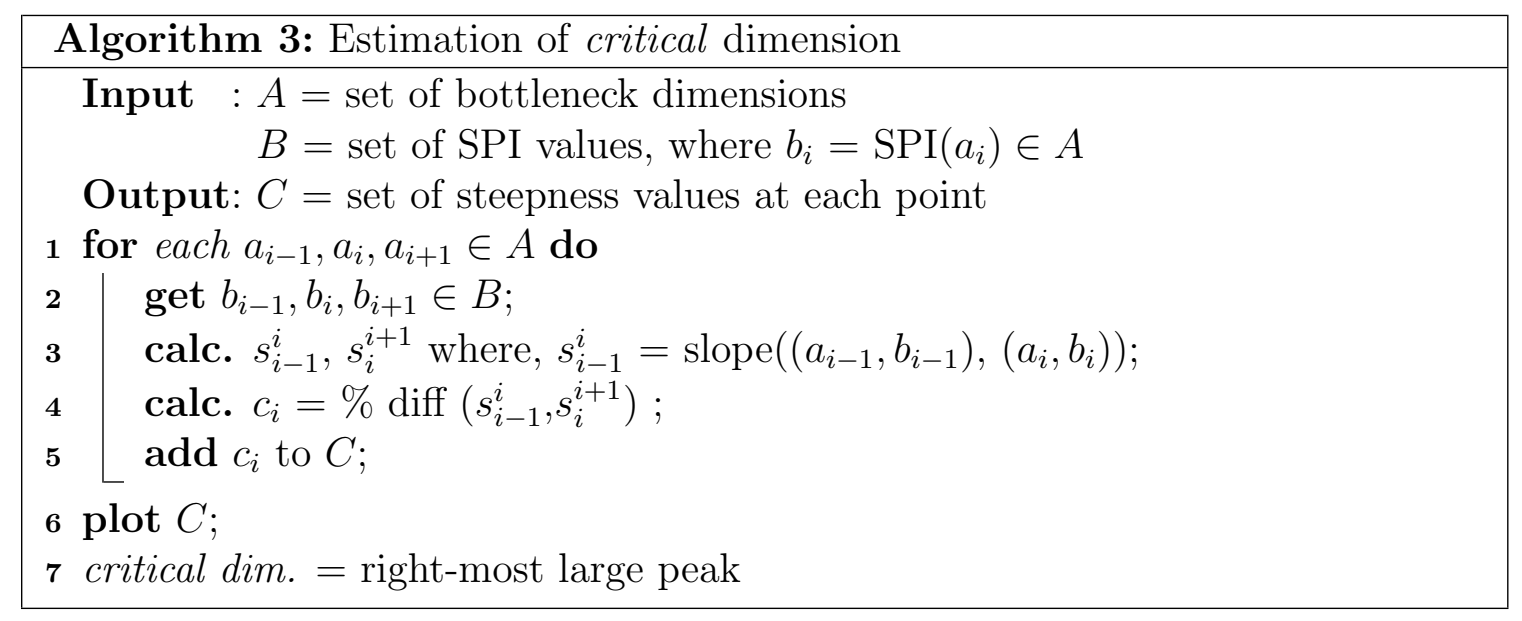

For providing a better graphical representation on how the critical bottleneck dimensionality is identified, Fig. 7.3 shows the second derivative approximation of the SPI curve. This is computed for all the different languages under consideration by using the proposed method. For some languages, there is a clear single peak where the SPI curve changes its behaviour drastically e.g. English, Spanish and Turkish. However, for some other languages, there exist multiple large peaks e.g. Russian and Arabic. In the latter cases, the right-most large peak is the one considered indicative of the critical bottleneck dimensionality. This is mainly because further below that point the network drastically loses the capacity for recovering the original data structure information.

Finally, it should be mentioned that the critical bottleneck dimensionality might 
English
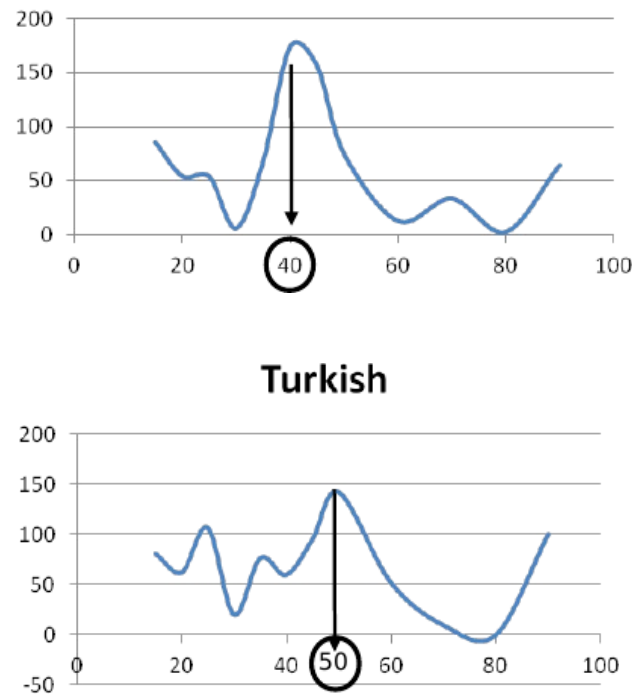

Spanish

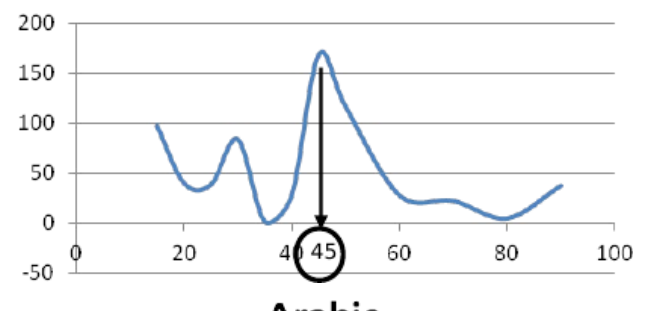

Arabic

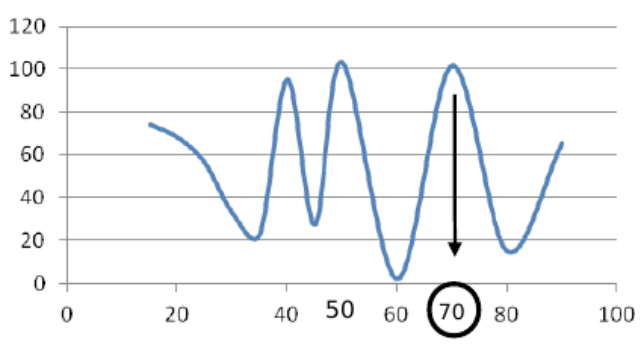

Russian

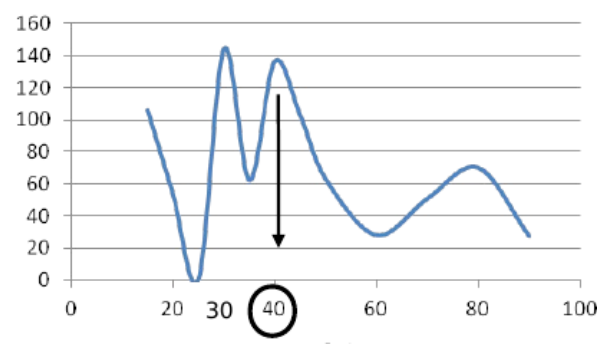

Figure 7.3: The percentage difference in slope of the SPI curve at each dimension.

not be easily spotted directly from the slope of the SPI curve, but plotting the percentage difference, which approximates the SPI's second derivative, clearly captures it. It is evident from the results presented in this section that different languages exhibit different critical bottleneck dimensionalities. This provides a much more principled criterion for the selection of the target dimensionalities in cross- and multi-language applications that use dimensionality reduction techniques. 


\subsection{Critical dimensionality and perplexity}

It has been discussed that the neocortex of the brain works in multiple layers where each layer captures some specific type of information (Quartz and Sejnowski, 1997; Utgoff and Stracuzzi, 2002). This presents a strong analogy to the computational deep learning framework. Inspired on this evidence, we anticipated that the critical bottleneck dimensionality of each language can be affected by their different structural and semantic characteristics.

We want to explore whether there is a relation between the grammatical complexity of a particular language and its critical dimensionality. According to this, and as an additional empirical analysis, we used the word trigram perplexities of each considered language as a proxy to its grammatical complexity, and we evaluated whether such a proxy correlates with the critical bottleneck dimensionalities obtained in the previous section.

Perplexity is often used as a metric for evaluating the quality of a language model. A word n-gram perplexity of value $V$ indicates that the considered model found $V$ alternatives for the following term; therefore, the better a model is, the lower the resulting perplexity. In the limit, the lowest perplexity achievable by a language model indicates the actual information content (entropy) of the given language (Brown et al., 1992).

\begin{tabular}{cccc}
\hline Lang. & Crit. Dim. & PPL-T & PPL-S \\
\hline en & 40 & 64.0018 & 59.6428 \\
es & 45 & 113.075 & 89.4268 \\
tk & 50 & 322.315 & 177.117 \\
ru & 40 & 218.634 & 159.588 \\
ar & 70 & 741.115 & 296.663 \\
\hline
\end{tabular}

Table 7.3: The word trigram perplexities for each language considering tokens (PPL-T) and stems (PPL-S) along with critical bottleneck dimensionality.

In order to establish whether the language information content and its critical bottleneck dimensionality correlate to each other, the Pearson's correlation coeffi- 
122 CHAPTER 7. BOTTLENECK DIMENSIONALITY FOR AUTOENCODERS

\begin{tabular}{lcc}
\hline Mode & Correlation & $p$-value \\
\hline tokens & 0.95797 & 0.10339 \\
stems & 0.88834 & $\mathbf{0 . 0 4 1 6 8}^{*}$ \\
\hline
\end{tabular}

Table 7.4: The correlation between critical dimensionality for a language and its word trigram perplexity. The $p$-value represents the two-tailed TTest values. * denotes the statistical significance $p<0.05$.

cient between the word trigram perplexity and the critical bottleneck dimensionalities obtained in the previous section is calculated. Table 7.3 presents the obtained perplexities for both, token and stem based, trigram models along with the critical bottleneck dimensionalities for each of the five languages under consideration; and Table 7.4 presents the resulting correlation coefficients and their corresponding $p$-values.

As observed from Table 7.4, although both correlation coefficients are high, only the correlation coefficient between the stem-based perplexity and the critical dimensionalities is statistically significant (this is not surprising as autoencoders were actually trained with stems rather than tokens). This result implies that there is actually a strong correspondence between the perplexity of a language and its critical bottleneck dimensionality. 

$\prod_{\text {Chapter }}$

\section{Conclusions \& future work}

In this Chapter we present the concluding remarks of the main finding of this dissertation (Section 8.1), discuss limitations (Section 8.2) and outline the potential future work (Section 8.4).

\subsection{Conclusions}

This dissertation deals with cross-view projection techniques for cross-view information retrieval tasks. In the exploration, a very important and prevalent problem of mixed-script IR is formally defined and investigated. The deep learning based neural cross-view models proposed in this dissertation provide state-of-the-art performance for various cross-language and cross-script applications. The dissertation also explored the architectural properties of the autoencoders which has attained less attention and establishes the notion of critical bottleneck dimensionality.

In this dissertation, the problem of mixed-script IR is introduced formally and motivated as a cross-view problem and the involved research challenges are presented. 
We also conducted a quantitative analysis on how much web search traffic is actually affected by MSIR through a large-scale empirical study of a commercial search engine query logs. This analysis has provided a lot of insight on the prevalence and impact of the MSIR behaviour on the web (RQ1). A principled solution to address the primary challenge of MSIR, the term variations across the scripts, is proposed in form of a cross-view autoencoder. The proposed mixed-script joint model learns abstract representation of terms across the scripts through deep learning architecture such that term equivalents are close to each other. The deep autoencoder based approach provides highly discriminative and powerful representation for terms with as low as 20 dimensions. An extensive empirical analysis is presented on a practical and important use-case: ad-hoc retrieval of songs lyrics. The experiments suggest that the state-of-the-art methods for handling spelling variation and transliteration mining have strong effect on the performance of IR in mixed-script space but the cross-view autoencoder significantly outperforms them (RQ1).

Cross-view autoencoders provide the best way to model terms across scripts because of the small and finite feature space as discussed in Section 4.1.2. They do not perform as strongly for modelling of cross-language documents which involve unbounded and large feature space. The external data composition neural network (XCNN) model, proposed in this dissertation, overcomes such limitations of the cross-view autoencoder for modelling cross-language text. We have presented and evaluated the XCNN framework on different cross-language tasks and found it to be statistically superior in performance to other strong baselines (RQ2). These two attributes of the XCNN model prove crucial for its performance in the retrieval tasks: (i) the learning framework proposed in this work gives a natural way to extend external relevance signals available in the form of pseudo relevance or clickthrough data to cross-language embeddings with the help of a small subset of parallel data, and (ii) the non-linear composition model optimises an objective function that directly relates to the considered task evaluation metric. These properties allow for the model to perform better than other latent semantic models which rely only on parallel data 
for training.

The gradient based learning provides a way to scale up to large training datasets more easily than linear methods that depend on matrix factorization, such as CL-LSI and OPCA. For XCNN, the time and space complexity grow linearly with the size of the vocabulary and the amount of training datapoints, while complexity grows quadratically for models based on matrix factorization. The XCNN model also outperforms the S2Net model, the only latent semantic model that optimises a loss function directly related to the evaluation metric. The use of non-linearity allows the model to learn interesting dependence between the terms across languages compared to their linear counterparts.

We have also explored a novel methodology to effectively include a deep learning based contextual similarity estimation, which handles source context for machine translation (RQ3). This feature is successfully incorporated in an end-to-end SMT system. The method shows statistically significant improvements compared to strong baseline systems in English-to-Spanish and English-to-Hindi translation tasks. Manual analysis confirmed the advantages of choosing the appropriate translation unit by taking into account the information of the input sentence context and the relation with the training sentences evidenced by the deep source context feature.

Finally, we have presented a comprehensive study on the use of autoencoders for modelling text data at the sentence level. Particularly, we explored the suitability of two different models, binary deep autoencoder (bDA) and replicated softmax deep autoencoder (rsDA), for constructing deep autoencoder representations of text data. In order to evaluate the quality of autoencoder generated representations, we defined and evaluated two novel metrics related to the reconstruction property of an autoencoder: structure preservation index (SPI) and similarity accumulation index (SAI). We also introduced the concept of critical bottleneck dimensionality (CBD) below which the structural information is lost for text representation with autoencoders. We have also proposed an automatic method to find the CBD using the SPI metric, which allows for a better discrimination and identification of CBD (RQ4). 
Our analysis of CBD across different languages has suggested there is a correlation between the critical bottleneck dimensionality and language perplexity.

\subsection{Limitations}

In this section we list the limitations of the methods presented in this dissertation.

Parallel/comparable data: Most of the models discussed in this dissertation assume that parallel or comparable data are available. In reality, such resources are very limited and almost non-existent for many languages. Small amounts of parallel data lead to poor performance for bilingual models especially in case of resource-poor languages. Although we have tried to address this issue in the XCNN model where it is initialised using monolingual data and then fine-tuned using small amount of cross-lingual data, such models are not applicable to language pairs for which no parallel data are available.

External relevance signals: In the XCNN model, we need external relevance signals such as relevance judgements or clickthrough data. The former is more expensive to obtain than the latter. Although we have tried to generate pseudo-relevance signals using standard retrieval models, they are not as effective as actual signals.

Computational resources: Training large neural networks on large datasets require heavy computational resources. Modelling all the terms present in the corpus is also a big challenge through neural networks because of enormously large and sparse visible layers. In order to perform such experiments, we used GPU's and assumed their availability. Although there are approaches like word-hashing where the terms are coded by their character n-grams to reduce the feature space, their suitability for cross-language models is still to be investigated. 
Mixed-script data: Being a relatively new area, there is negligible amount of public data available for mixed-script IR. We have developed the first such datasets for Hindi and made available through FIRE shared task on mixed-script IR (Saha Roy et al., 2013; Choudhury et al., 2014). Similarly, the training data for modelling mixed-script terms is also very scarce. Although traditional transliteration data are available, they do not represent the spelling variations within the script well. The CAE model assumes the availability of such data.

Evaluation metrics for mixed-script terms: Currently MSIR is being evaluated by the quality of retrieval in ad-hoc retrieval setting. We believe there is a need to device evaluation metrics which capture how close is the transliterated query term to the actual term in order to allow qualitative analysis.

\subsection{Code}

In order to promote replicability, we have made the code publicly available as much as possible. The code also contains details on parameter tuning details. The code related to cross-view autoencoder for mixed-script IR is available at: http://users. dsic.upv.es/ pgupta/mixed-script-ir.html, the code for XCNN is available at: https://github.com/parthg/jDNN

\subsection{Future work}

\subsubsection{Mixed-script IR}

In this dissertation, we have conducted some initial research in the emerging area of mixed-script IR. Future work in this area must also deal with the related problem of code-mixing in queries and documents. Code-mixing is a growing phenomenon in the user-generated content and provide additional challenges for MSIR. Similarly, one 
should also extend the MSIR framework to a more general setup such as mixed-script multilingual IR.

\subsubsection{Composition neural networks}

The external relevance composition neural network (XCNN) model provides a stateof-the-art performance for many CLIR applications. This model provides a natural way of incorporating semantic compositional models. One can extend this model with such techniques to obtain richer representation of text data depending on the requirement of the application. We believe such techniques may provide very effective performance for more sophisticated tasks such as semantic textual entailment compared to information retrieval.

\subsubsection{Source context features}

Interesting future work on source context features using deep autoencoder as presented in this work should focus on better integrating the dynamic feature into translation decoding at the architecture level. In its current form, it lies as an additional layer to the existing phrase-based machine translation system. This also increases the search time. To speed-up search, one can divide the feature space in chunks and search hierarchically, perform clustering or use kd-trees like data structures.

\subsubsection{Qualitative metrics}

One possible extension of our study on suitability of the proposed metrics, especially SPI, should focus on using the proposed metrics as error metrics during the autoencoder fine tuning stage. If this metric can be used along with back-propagation, we envisage a new generation of text-oriented autoencoders that will be able to provide a much better characterization of the linguistic phenomenon in text data. Another future extension will be to validate the impact of the proposed metrics using extrinsic evaluation. 


\subsubsection{More applications}

We also want to extend the models from this thesis to more cross-view applications

such as: ( $i$ ) enriching bilingual dictionaries (Dubey et al., 2014); (ii) discovering parallel and reused text in news stories (Gupta et al., 2013b); and (iii) cross-language text categorisation (Klementiev et al., 2012). 



\section{Bibliography}

Adafre, S. F. and de Rijke, M. (2006). Finding similar sentences across multiple languages in wikipedia. In Proceedings of the EACL Workshop on New Text: Wikis and Blogs and Other Dynamic Text Sources, EACL '06, pages 62-69.

Ahmed, U. Z., Bali, K., Choudhury, M., and VB, S. (2011). Challenges in designing input method editors for indian lan-guages: The role of word-origin and context. In Proceedings of the Workshop on Advances in Text Input Methods, WTIM '11, pages 1-9.

Amati, G. (2006). Frequentist and bayesian approach to information retrieval. In Proceedings of the 28th European Conference on Advances in Information Retrieval, ECIR'06, pages 13-24, Berlin, Heidelberg. Springer-Verlag.

Anderka, M. and Stein, B. (2009). The esa retrieval model revisited. In Proceedings of the 32Nd International ACM SIGIR Conference on Research and Development in Information Retrieval, SIGIR '09, pages 670-671, New York, NY, USA. ACM.

Banchs, R. E. and Costa-jussà, M. R. (2011). A semantic feature for statistical machine translation. In Proceedings of the Fifth Workshop on Syntax, Semantics and Structure in Statistical Translation, SSST-5, pages 126-134.

Banchs, R. E. and Kaltenbrunner, A. (2008). Exploiting mds projections for cross- 
language ir. In Proceedings of the 31st Annual International ACM SIGIR Conference on Research and Development in Information Retrieval, SIGIR '08, pages 863-864, New York, NY, USA. ACM.

Banerjee, S. and Pedersen, T. (2003). Extended gloss overlaps as a measure of semantic relatedness. In Proceedings of the 18th International Joint Conference on Artificial Intelligence, IJCAI'03, pages 805-810, San Francisco, CA, USA. Morgan Kaufmann Publishers Inc.

Bar-Yossef, Z. and Kraus, N. (2011). Context-sensitive query auto-completion. In Proceedings of the 20th International Conference on World Wide Web, WWW '11, pages 107-116, New York, NY, USA. ACM.

Barrón-Cedeño, A., Rosso, P., Agirre, E., and Labaka, G. (2010). Plagiarism detection across distant language pairs. In Proceedings of the 23rd International Conference on Computational Linguistics, COLING '10, pages 37-45, Stroudsburg, PA, USA. Association for Computational Linguistics.

Barrón-Cedeño, A. (2012). On the mono- and cross-language detection of text re-use and plagiarism. Ph.D. thesis, Universitat Politènica de València.

Barrón-Cedeño, A., Rosso, P., Pinto, D., and Juan, A. (2008). On cross-lingual plagiarism analysis using a statistical model. In Proceedings of the Workshop on Uncovering Plagiarism, Authorship and Social Software Misuse, ECAI'08.

Barrón-Cedeño, A., Gupta, P., and Rosso, P. (2013). Methods for cross-language plagiarism detection. Knowledge-Based Systems, 50, 211-217.

Bast, H. and Weber, I. (2006). Type less, find more: Fast autocompletion search with a succinct index. In Proceedings of the 29th Annual International ACM SIGIR Conference on Research and Development in Information Retrieval, SIGIR '06, pages 364-371, New York, NY, USA. ACM. 
Blei, D. M., Ng, A. Y., and Jordan, M. I. (2003). Latent dirichlet allocation. Journal of Machine Learning Research, 3, 993-1022.

Bojar, O., Buck, C., Federmann, C., Haddow, B., Koehn, P., Leveling, J., Monz, C., Pecina, P., Post, M., Saint-Amand, H., Soricut, R., Specia, L., and Tamchyna, A. (2014a). Findings of the 2014 workshop on statistical machine translation. In Proceedings of the Ninth Workshop on Statistical Machine Translation, pages 12-58, Baltimore, Maryland, USA. Association for Computational Linguistics.

Bojar, O., Diatka, V., Rychly, P., Stranak, P., Suchomel, V., Tamchyna, A., and Zeman, D. (2014b). Hindencorp - hindi-english and hindi-only corpus for machine translation. In Proceedings of the Ninth International Conference on Language Resources and Evaluation, LREC'14, Reykjavik, Iceland.

Bourlard, H. and Kamp, Y. (1988). Auto-association by multilayer perceptrons and singular value decomposition. Biological Cybernetics, 59(4), 291-294.

Braschler, M. (2004). Combination approaches for multilingual text retrieval. Information Retrieval, 7(1-2), 183-204.

Braschler, M., Krause, J., Peters, C., and Schäuble, P. (1998). Cross-language information retrieval (CLIR) track overview. In Proceedings of The Seventh Text REtrieval Conference, TREC 1998, Gaithersburg, Maryland, USA, November 911, 1998, pages $1-8$.

Braschler, M., Schäuble, P., and Peters, C. (1999). Cross-language information retrieval (CLIR) track overview. In Proceedings of The Eighth Text REtrieval Conference, TREC 1999, Gaithersburg, Maryland, USA, November 17-19, 1999.

Bromley, J., Guyon, I., LeCun, Y., Säckinger, E., and Shah, R. (1993). Signature verification using a siamese time delay neural network. In Proceedings of Advances in Neural Information Processing Systems Conference, Denver, Colorado, USA, NIPS' 93, pages 737-744. 
Brown, P. F., Pietra, V. J. D., Mercer, R. L., Pietra, S. A. D., and Lai, J. C. (1992). An estimate of an upper bound for the entropy of english. Comput. Linguist., 18(1), 31-40.

Burges, C. (2010). Dimension reduction: A guided tour. Foundations and Trends in Machine Learning.

Carterette, B., Kanoulas, E., Hall, M. M., and Clough, P. D. (2014). Overview of the TREC 2014 session track. In Proceedings of The Twenty-Third Text REtrieval Conference, TREC 2014, Gaithersburg, Maryland, USA, November 19-21, 2014.

Ceska, Z., Toman, M., and Jezek, K. (2008). Multilingual Plagiarism Detection. In Proceedings of the 13th International Conference on Artificial Intelligence (ICAI 2008), pages 83-92, Varna, Bulgaria. Springer-Verlag.

Chandar A. P., S., Lauly, S., Larochelle, H., Khapra, M. M., Ravindran, B., Raykar, V. C., and Saha, A. (2014). An autoencoder approach to learning bilingual word representations. In Advances in Neural Information Processing Systems 27: Annual Conference on Neural Information Processing Systems 2014, December 8-13 2014, Montreal, Quebec, Canada, pages 1853-1861.

Chen, H.-H., Hueng, S.-J., Ding, Y.-W., and Tsai, S.-C. (1998). Proper name translation in cross-language information retrieval. In Proceedings of the 36th Annual Meeting of the Association for Computational Linguistics and 17th International Conference on Computational Linguistics - Volume 1, ACL '98, pages 232-236, Stroudsburg, PA, USA. Association for Computational Linguistics.

Choudhury, M., Bali, K., Gupta, K., and Datha, N. (2012). Multilingual search for transliterated content. Patent number US 20120278302.

Choudhury, M., Chittaranjan, G., Gupta, P., and Das, A. (2014). Overview of FIRE 2014 track on transliterated search. In Sixth Forum for Information Retrieval Evaluation, FIRE '14. 
Church, K. (1993). Char_align: A Program for Aligning Parallel Texts at the Character Level. In Proceedings of the 31st Annual Meeting of the Association for Computational Linguistics, ACL '93, pages 1-8, Columbus, OH, USA. Association for Computational Linguistics.

Costa-jussà, M. R., Gupta, P., Rosso, P., and Banchs, R. E. (2014). English-to-hindi system description for WMT 2014: Deep source-context features for Moses. In Proceedings of the Ninth Workshop on Statistical Machine Translation, WMT '14, pages 79-83, Baltimore, Maryland, USA. Association for Computational Linguistics.

Cox, T. F. and Cox, M. (2000). Multidimensional Scaling. CRC Press, Boca Raton, FL, USA.

Dash, M. and Liu, H. (1997). Feature selection for classification. Intelligent Data Analysis, 1, 131-156.

Deerwester, S. C., Dumais, S. T., Landauer, T. K., Furnas, G. W., and Harshman, R. A. (1990). Indexing by latent semantic analysis. JASIS, 41(6), 391-407.

Diamantaras, K. I. and Kung, S. Y. (1996). Principal Component Neural Networks: Theory and Applications. John Wiley \& Sons, Inc., New York, NY, USA.

Dua, N., Gupta, K., Choudhury, M., and Bali, K. (2011). Query completion without query logs for song search. In Proceedings of $W W W$ (Companion Volume), pages $31-32$.

Dubey, A., Gupta, P., Varma, V., and Rosso, P. (2014). Enrichment of bilingual dictionary through news stream data. In Proceedings of the Ninth International Conference on Language Resources and Evaluation, LREC '14, pages 3761-3765.

Dumais, S., Landauer, T. K., and Littman, M. L. (1997). Automatic Cross-Linguistic Information Retrieval using Latent Semantic Indexing. In AAAI-97 Spring Symposium Series: Cross-Language Text and Speech Retrieval, pages 18-24. 
Durrani, N., Haddow, B., Koehn, P., and Heafield, K. (2014). Edinburgh's phrasebased machine translation systems for WMT-14. In Proceedings of the Ninth Workshop on Statistical Machine Translation, WMT '14, Baltimore, Maryland, USA. Association for Computational Linguistics.

Efthimiadis, E. N. (2008). How do greeks search the web?: A query log analysis study. In Proceedings of iNEWS, pages 81-84.

Efthimiadis, E. N., Malevris, N., Kousaridas, A., Lepeniotou, A., and Loutas, N. (2009). Non-english web search: an evaluation of indexing and searching the greek web. Information Retrieval, 12(3).

Erhan, D., Bengio, Y., Courville, A., Manzagol, P.-A., Vincent, P., and Bengio, S. (2010). Why does unsupervised pre-training help deep learning? Journal of Machine Learning Research, 11, 625-660.

Fellbaum, C. (1998). WordNet: An electronic lexical database. Bradford Books.

Fodor, I. (2002). A survey of dimension reduction techniques. Technical report, LLNL technical report, UCRL ID-148494.

Franco-Salvador, M., Gupta, P., and Rosso, P. (2013). Cross-language plagiarism detection using a multilingual semantic network. In Proceedings of the 35th European Conference on Advances in Information Retrieval, ECIR'13, pages 710-713, Berlin, Heidelberg. Springer-Verlag.

Franco-Salvador, M., Rosso, P., and Navigli, R. (2014). A knowledge-based representation for cross-language document retrieval and categorization. In Proceedings of the 14th Conference of the European Chapter of the Association for Computational Linguistics, pages 414-423. Association for Computational Linguistics.

Franco-Salvador, M., Gupta, P., Rosso, P., and Banchs, R. E. (2016). Cross-language plagiarism detection over continuous-space- and knowledge graph-based representations of language. Knowledge-Based Systems, 111, 87-98. 
Franco-Salvador, M., Rosso, P., and Montes-y-Gómez, M. (2016). A systematic study of knowledge graph analysis for cross-language plagiarism detection. Information Processing \& Management, 52(4), 550-570.

French, J. C., Powell, A. L., and Schulman, E. (1997). Applications of approximate word matching in information retrieval. In Proceedings of the Sixth International Conference on Information and Knowledge Management, CIKM '97, pages 9-15, New York, NY, USA. ACM.

Fung, P. and Cheung, P. (2004). Multi-level bootstrapping for extracting parallel sentences from a quasi-comparable corpus. In Proceedings of the 20th International Conference on Computational Linguistics, COLING '04, Stroudsburg, PA, USA. Association for Computational Linguistics.

Gabrilovich, E. and Markovitch, S. (2007). Computing semantic relatedness using wikipedia-based explicit semantic analysis. In Proceedings of the 20th international joint conference on Artifical intelligence, IJCAI'07, pages 1606-1611, San Francisco, CA, USA. Morgan Kaufmann Publishers Inc.

Gao, J., Nie, J.-Y., Xun, E., Zhang, J., Zhou, M., and Huang, C. (2001). Improving query translation for cross-language information retrieval using statistical models. In Proceedings of the 24th Annual International ACM SIGIR Conference on Research and Development in Information Retrieval, SIGIR '01, pages 96-104, New York, NY, USA. ACM.

Gao, J., He, X., and Nie, J.-Y. (2010). Clickthrough-based translation models for web search: From word models to phrase models. In Proceedings of the 19th ACM International Conference on Information and Knowledge Management, CIKM '10, pages 1139-1148, New York, NY, USA. ACM.

Gao, J., Toutanova, K., and Yih, W.-t. (2011). Clickthrough-based latent semantic models for web search. In Proceedings of the 34 th International ACM SIGIR 
Conference on Research and Development in Information Retrieval, SIGIR '11, pages 675-684, New York, NY, USA. ACM.

Gehler, P. V., Holub, A. D., and Welling, M. (2006). The rate adapting poisson model for information retrieval and object recognition. In Proceedings of the 23rd International Conference on Machine Learning, ICML '06, pages 337-344, New York, NY, USA. ACM.

Geng, X., Liu, T.-Y., Qin, T., and Li, H. (2007). Feature selection for ranking. In Proceedings of the 30th Annual International ACM SIGIR Conference on Research and Development in Information Retrieval, SIGIR '07, pages 407-414, New York, NY, USA. ACM.

Gupta, K., Choudhury, M., and Bali, K. (2012a). Mining Hindi-English transliteration pairs from online Hindi lyrics. In Proceedings of the Eighth International Conference on Language Resources and Evaluation, LREC'12, pages 2459-2465.

Gupta, P. (2014). Modelling of terms across scripts through autoencoders. In Proceedings of the 37th International ACM SIGIR Conference on Research 85 Development in Information Retrieval, SIGIR '14, pages 1279-1279, New York, NY, USA. ACM.

Gupta, P. and Rosso, P. (2012a). Expected divergence based feature selection for learning to rank. In Proceedings of the 24th International Conference on Computational Linguistics, COLING' 12, pages 431-440.

Gupta, P. and Rosso, P. (2012b). Text reuse with ACL: (Upward) Trends. In Proceedings of the ACL-2012 Special Workshop on Rediscovering 50 Years of Discoveries, ACL '12, pages 76-82, Stroudsburg, PA, USA. Association for Computational Linguistics.

Gupta, P., Barrón-Cedeño, A., and Rosso, P. (2012b). Cross-language high similarity search using a conceptual thesaurus. In Proceedings of the Third International 
Conference on Information Access Evaluation: Multilinguality, Multimodality, and Visual Analytics, CLEF '12, pages 67-75, Berlin, Heidelberg. Springer-Verlag.

Gupta, P., Rosso, P., and Banchs, R. E. (2013a). Encoding transliteration variation through dimensionality reduction: FIRE Shared Task on Transliterated Search. In Fifth Forum for Information Retrieval Evaluation, FIRE '13.

Gupta, P., Clough, P. D., Rosso, P., Stevenson, M., and Banchs, R. E. (2013b). PAN@FIRE: Overview of the Cross-Language !ndian News Story Search (CL!NSS) track. In Fifth Forum for Information Retrieval Evaluation, FIRE '13.

Gupta, P., Bali, K., Banchs, R. E., Choudhury, M., and Rosso, P. (2014). Query expansion for mixed-script information retrieval. In Proceedings of the 37th International ACM SIGIR Conference on Research $\&$ Development in Information Retrieval, SIGIR '14, pages 677-686, New York, NY, USA. ACM.

Gupta, P., Banchs, R. E., and Rosso, P. (2016a). Continuous space models for CLIR. Information Processing \&3 Management, 53(2), 359-370.

Gupta, P., Costa-Jussà, M. R., Rosso, P., and Banchs, R. E. (2016b). A deep sourcecontext feature for lexical selection in statistical machine translation. Pattern Recognition Letters, 75, 24-29.

Gupta, P., Banchs, R. E., and Rosso, P. (2016c). Squeezing bottlenecks: Exploring the limits of autoencoder semantic representation capabilities. Neurocomputing, 175, 1001-1008.

Guyon, I. and Elisseeff, A. (2003). An introduction to variable and feature selection. Journal of Machine Learning Research, 3, 1157-1182.

Hall, P. A. V. and Dowling, G. R. (1980). Approximate string matching. ACM Computing Surveys, 12(4), 381-402. 
Hassan, S. and Mihalcea, R. (2011). Semantic relatedness using salient semantic analysis. In Proceedings of the Twenty-Fifth AAAI Conference on Artificial Intelligence, AAAI'11, pages 884-889. AAAI Press.

Hermann, K. M. and Blunsom, P. (2013). The role of syntax in vector space models of compositional semantics. In Proceedings of the 51st Annual Meeting of the Association for Computational Linguistics, ACL 2013, 4-9 August 2013, Sofia, Bulgaria, Volume 1: Long Papers, pages 894-904.

Hermann, K. M. and Blunsom, P. (2014). Multilingual models for compositional distributed semantics. In Proceedings of the 52nd Annual Meeting of the Association for Computational Linguistics, ACL 2014, June 22-27, 2014, Baltimore, MD, USA, Volume 1: Long Papers, pages 58-68.

Hestenes, M. R. and Stiefel, E. (1952). Methods of conjugate gradients for solving linear systems. Journal of research of the National Bureau of Standards, 49, 409436.

Hinton, G. and Salakhutdinov, R. (2006). Reducing the dimensionality of data with neural networks. Science, 313(5786), $504-507$.

Hinton, G. E. (2002). Training products of experts by minimizing contrastive divergence. Neural Computation, 14(8), 1771-1800.

Hofmann, T. (1999). Probabilistic latent semantic indexing. In Proceedings of the 22nd annual international ACM SIGIR conference on Research and development in information retrieval, SIGIR '99, pages 50-57, New York, NY, USA. ACM.

Hollink, V., Kamps, J., Monz, C., and De Rijke, M. (2004). Monolingual document retrieval for european languages. Inf. Retr., 7(1-2), 33-52.

Huang, P.-S., He, X., Gao, J., Deng, L., Acero, A., and Heck, L. (2013). Learning deep structured semantic models for web search using clickthrough data. In Pro- 
ceedings of the 22Nd ACM International Conference on Conference on Information E\#38; Knowledge Management, CIKM '13, pages 2333-2338, New York, NY, USA. ACM.

Jackson, D. A., Somers, K. M., and Harvey, H. H. (1989). Similarity coefficients:measures of co-occurrence and association or simply measures of occurrence. The American Naturalist, 133(3), 436-453.

Janarthanam, S. C., Subramaniam, S., and Nallasamy, U. (2008). Named entity transliteration for cross-language information retrieval using compressed word format mapping algorithm. In Proceedings of iNEWS, pages 33-38.

Janecek, A. G., Gansterer, W. N., Demel, M. A., and Ecker, G. F. (2008). On the Relationship Between Feature Selection and Classification Accuracy. In JMLR: Workshop and Conference Proceedings 4, pages 90-105.

Järvelin, K. and Kekäläinen, J. (2002). Cumulated gain-based evaluation of IR techniques. ACM Transactions on Information Systems, 20(4), 422-446.

King, B. and Abney, S. P. (2013). Labeling the languages of words in mixed-language documents using weakly supervised methods. In Proceedings of Human Language Technologies: Conference of the North American Chapter of the Association of Computational Linguistics, HLT-NAACL '13, pages 1110-1119.

Klementiev, A., Titov, I., and Bhattarai, B. (2012). Inducing crosslingual distributed representations of words. In Proceedings of the 24th International Conference on Computational Linguistics, COLING '12, pages 1459-1474.

Knight, K. and Graehl, J. (1998). Machine transliteration. Comput. Linguist., 24(4), 599-612.

Koehn, P., Hoang, H., Birch, A., Callison-Burch, C., Federico, M., Bertoldi, N., Cowan, B., Shen, W., Moran, C., Zens, R., Dyer, C., Bojar, O., Constantin, 
A., and Herbst, E. (2007). Moses: Open source toolkit for statistical machine translation. In Proceedings of the 45th Annual Meeting of the ACL on Interactive Poster and Demonstration Sessions, ACL '07, pages 177-180, Stroudsburg, PA, USA. Association for Computational Linguistics.

Kumar, S. and Udupa, R. (2011). Learning hash functions for cross-view similarity search. In Proceedings of the Twenty-Second International Joint Conference on Artificial Intelligence - Volume Volume Two, IJCAI'11, pages 1360-1365. AAAI Press.

Kumaran, A., Khapra, M. M., and Li, H. (2010). Report of news 2010 transliteration mining shared task. In Proceedings of NEWS, pages 21-28.

Lauly, S., Boulanger, A., and Larochelle, H. (2014). Learning multilingual word representations using a bag-of-words autoencoder. CoRR, abs/1401.1803.

Lazarinis, F., Ferro, J. V., and Tait, J. (2007). Improving non-english web searching (inews07). SIGIR Forum, 41(2), 72-76.

LeCun, Y., Chopra, S., Hadsell, R., Ranzato, M., and Huang, F. (2006). A tutorial on energy-based learning. In G. Bakir, T. Hofman, B. Schölkopf, A. Smola, and B. Taskar, editors, Predicting Structured Data. MIT Press.

Littman, M., Dumais, S., and Landauer, T. (1998). Cross-Language Information Retrieval, chapter 5, chapter Automatic Cross-language Information Retrieval Using Latent Semantic Indexing, pages 51-62. Kluwer Academic Publishers.

Liu, S., Yu, C., and Meng, W. (2005). Word sense disambiguation in queries. In Proceedings of the 14 th ACM International Conference on Information and Knowledge Management, CIKM '05, pages 525-532, New York, NY, USA. ACM.

Manning, C. D. and Schütze, H. (1999). Foundations of Statistical Natural Language Processing. MIT Press, Cambridge, MA, USA. 
Manning, C. D., Raghavan, P., and Schütze, H. (2008). Introduction to Information Retrieval. Cambridge University Press, New York, NY, USA.

Mcnamee, P. and Mayfield, J. (2004). Character n-gram tokenization for European language text retrieval. Information Retrieval, 7(1), 73-97.

Mikolov, T., Le, Q. V., and Sutskever, I. (2013). Exploiting similarities among languages for machine translation. CoRR, abs/1309.4168.

Mimno, D. M., Wallach, H. M., Naradowsky, J., Smith, D. A., and McCallum, A. (2009). Polylingual topic models. In Proceedings of the 2009 Conference on Empirical Methods in Natural Language Processing, EMNLP 2009, 6-7 August 2009, Singapore, A meeting of SIGDAT, a Special Interest Group of the ACL, pages $880-889$.

Mohamed, A., Dahl, G., and Hinton, G. (2012). Acoustic modeling using deep belief networks. IEEE Transactions on Audio, Speech, and Language Processing, 20(1), $14-22$.

Munteanu, D. S. and Marcu, D. (2005). Improving machine translation performance by exploiting non-parallel corpora. Comput. Linguist., 31(4), 477-504.

Navigli, R. and Ponzetto, S. P. (2012). BabelNet: The automatic construction, evaluation and application of a wide-coverage multilingual semantic network. Artificial Intelligence, 193, 217-250.

Nocedal, J. (1980). Updating Quasi-Newton Matrices with Limited Storage. Mathematics of Computation, 35(151), 773-782.

Oard, D. W., Levow, G.-A., and Cabezas, C. I. (2001). Clef experiments at maryland: Statistical stemming and backoff translation. In Revised Papers from the Workshop of Cross-Language Evaluation Forum on Cross-Language Information Retrieval and Evaluation, CLEF '00, pages 176-187, London, UK. Springer-Verlag. 
Och, F. J. and Ney, H. (2003). A systematic comparison of various statistical alignment models. Computational Linguistics, 29(1), 19-51.

Pal, D., Majumder, P., Mitra, M., Mitra, S., and Sen, A. (2008). Issues in searching for indian language web content. In Proceedings of $i N E W S$, pages 93-96.

Palchowdhury, S., Majumder, P., Pal, D., Bandyopadhyay, A., and Mitra, M. (2011). Overview of FIRE 2011. In Third Forum for Information Retrieval Evaluation, FIRE '11, pages $1-12$.

Papineni, K., Roukos, S., Ward, T., and Zhu, W.-J. (2002). Bleu: A method for automatic evaluation of machine translation. In Proceedings of the 40th Annual Meeting on Association for Computational Linguistics, ACL '02, pages 311-318.

Platt, J. C., Toutanova, K., and Yih, W.-t. (2010). Translingual document representations from discriminative projections. In Proceedings of the 2010 Conference on Empirical Methods in Natural Language Processing, EMNLP '10, pages 251-261, Stroudsburg, PA, USA. Association for Computational Linguistics.

Potthast, M., Stein, B., and Anderka, M. (2008). A wikipedia-based multilingual retrieval model. In Proceedings of the IR Research, 30th European Conference on Advances in Information Retrieval, ECIR'08, pages 522-530, Berlin, Heidelberg. Springer-Verlag.

Potthast, M., Stein, B., Eiselt, A., Barrón-Cedeño, A., and Rosso, P. (2009). Overview of the 1 st international competition on plagiarism detection. volume 502, pages 1-9, San Sebastian, Spain. CEUR-WS.org. http://ceur-ws.org/Vol-502.

Potthast, M., Stein, B., Barrón-Cedeño, A., and Rosso, P. (2010). An evaluation framework for plagiarism detection. In Proceedings of the 23rd international conference on computational linguistics: Posters, COLING '10, pages 997-1005. Association for Computational Linguistics. 
Potthast, M., Barrón-Cedeño, A., Stein, B., and Rosso, P. (2011). Cross-language plagiarism detection. Language Resources and Evaluation (LRE), Special Issue on Plagiarism and Authorship Analysis, 45(1), 1-18.

Potthast, M., Gollub, T., Hagen, M., Kiesel, J., Michel, M., Oberländer, A., Tippmann, M., Barrón-Cedeño, A., Gupta, P., Rosso, P., and Stein, B. (2012). Overview of the 4 th international competition on plagiarism detection. In CLEF Evaluation Labs and Workshop, Online Working Notes, CLEF' '12.

Pouliquen, B., Steinberger, R., and Ignat, C. (2003). Automatic Identification of Document Translations in Large Multilingual Document Collections. In Proceedings of the International Conference on Recent Advances in Natural Language Processing (RANLP-2003), pages 401-408, Borovets, Bulgaria.

Qu, Y., Grefenstette, G., and Evans, D. A. (2003). Automatic transliteration for japanese-to-english text retrieval. In Proceedings of the 26th Annual International ACM SIGIR Conference on Research and Development in Information Retrieval, SIGIR '03, pages 353-360, New York, NY, USA. ACM.

Quartz, S. R. and Sejnowski, T. J. (1997). The neural basis of cognitive development: A constructivist manifesto. Behavioral and Brain Sciences, 20(04).

Raj, A. A. and Maganti, H. (2009). Transliteration based search engine for multilingual information access. In Proceedings of CLIAWS3, pages 12-20.

Raman, K., Bennett, P. N., and Collins-Thompson, K. (2013). Toward whole-session relevance: Exploring intrinsic diversity in web search. In Proceedings of the 36th International ACM SIGIR Conference on Research and Development in Information Retrieval, SIGIR '13, pages 463-472, New York, NY, USA. ACM.

Rocchio, J. J. (1971). Relevance feedback in information retrieval. In G. Salton, editor, The Smart retrieval system - experiments in automatic document processing, pages 313-323. Englewood Cliffs, NJ: Prentice-Hall. 
Rumelhart, D. E., Hinton, G. E., and Williams, R. J. (1986). Learning representations by back-propagating errors. Nature, 323, 533 - 536 .

Saha Roy, R., Choudhury, M., Majumder, P., and Agarwal, K. (2013). Overview and Datasets of FIRE 2013 Track on Transliterated Search. In Fifth Forum for Information Retrieval Evaluation, FIRE' '13.

Salakhutdinov, R. and Hinton, G. (2009a). Replicated softmax: An undirected topic model. In Proceedings of the 22Nd International Conference on Neural Information Processing Systems, NIPS'09, pages 1607-1614, USA. Curran Associates Inc.

Salakhutdinov, R. and Hinton, G. (2009b). Semantic hashing. International Journal of Approximate Reasoning, 50(7), 969-978.

Salakhutdinov, R., Mnih, A., and Hinton, G. (2007). Restricted boltzmann machines for collaborative filtering. In Proceedings of the 24th International Conference on Machine Learning, ICML '07, pages 791-798, New York, NY, USA. ACM.

Salton, G., Fox, E. A., and Wu, H. (1983). Extended boolean information retrieval. Communications of the ACM, 26(11), 1022-1036.

Sanderson, M. (1994). Word sense disambiguation and information retrieval. In Proceedings of the 17th Annual International ACM SIGIR Conference on Research and Development in Information Retrieval, SIGIR '94, pages 142-151, New York, NY, USA. Springer-Verlag New York, Inc.

Sequiera, R., Choudhury, M., Gupta, P., Rosso, P., Kumar, S., Banerjee, S., Naskar, S. K., Bandyopadhyay, S., Chittaranjan, G., Das, A., and Chakma, K. (2015). Overview of FIRE-2015 shared task on mixed script information retrieval. In Seventh Forum for Information Retrieval Evaluation, FIRE '15, pages 19-25.

Simard, M., Foster, G. F., and Isabelle, P. (1992). Using cognates to align sentences in bilingual corpora. In Proceedings of the Fourth International Conference on Theoretical and Methodological Issues in Machine Translation. 
Singhal, A., Buckley, C., and Mitra, M. (1996). Pivoted document length normalization. In Proceedings of the 19th Annual International ACM SIGIR Conference on Research and Development in Information Retrieval, SIGIR '96, pages 21-29, New York, NY, USA. ACM.

Socher, R., Pennington, J., Huang, E. H., Ng, A. Y., and Manning, C. D. (2011). Semi-supervised recursive autoencoders for predicting sentiment distributions. In Proceedings of the Conference on Empirical Methods in Natural Language Processing, EMNLP '11, pages 151-161, Stroudsburg, PA, USA. Association for Computational Linguistics.

Socher, R., Huval, B., Manning, C. D., and Ng, A. Y. (2012). Semantic compositionality through recursive matrix-vector spaces. In Proceedings of the 2012 Joint Conference on Empirical Methods in Natural Language Processing and Computational Natural Language Learning, EMNLP-CoNLL '12, pages 1201-1211, Stroudsburg, PA, USA. Association for Computational Linguistics.

Sowmya, V. B. and Varma, V. (2009). Transliteration based text input methods for telugu. In Proceedings of ICCPOL, pages 122-132.

Stein, B., Meyer zu Eissen, S., and Potthast, M. (2007). Strategies for Retrieving Plagiarized Documents. In C. Clarke, N. Fuhr, N. Kando, W. Kraaij, and A. de Vries, editors, Proceedings of the 30th Annual International ACM SIGIR Conference on Research and Development in Information Retrieval, pages 825-826, Amsterdam, The Netherlands. ACM Press.

Steinberger, R., Pouliquen, B., and Hagman, J. (2002). Cross-lingual Document Similarity Calculation Using the Multilingual Thesaurus EUROVOC. Computational Linguistics and Intelligent Text Processing. Proceedings of the CICLing 2002, LNCS (2276), 415-424. Springer-Verlag.

Steinberger, R., Pouliquen, B., Widiger, A., Ignat, C., Erjavec, T., Tufis, D., and Varga, D. (2006). The jrc-acquis: A multilingual aligned parallel corpus with +20 
languages. In Proceedings of Fifth International Conference on Language Resources and Evaluation, LREC'06.

Ture, F. and Lin, J. (2014). Exploiting representations from statistical machine translation for cross-language information retrieval. ACM Transactions on Information Systems, 32(4), 19:1-19:32.

Türe, F. and Lin, J. J. (2012). Why not grab a free lunch? mining large corpora for parallel sentences to improve translation modeling. In Human Language Technologies: Conference of the North American Chapter of the Association of Computational Linguistics, Proceedings, June 3-8, 2012, Montréal, Canada, pages 626-630.

Udupa, R. and Khapra, M. (2010a). Improving the multilingual user experience of wikipedia using cross-language name search. In Human Language Technologies: The 2010 Annual Conference of the North American Chapter of the Association for Computational Linguistics, HLT '10, pages 492-500, Stroudsburg, PA, USA. Association for Computational Linguistics.

Udupa, R. and Khapra, M. M. (2010b). Transliteration equivalence using canonical correlation analysis. In Proceedings of the 32Nd European Conference on Advances in Information Retrieval, ECIR'2010, pages 75-86, Berlin, Heidelberg. SpringerVerlag.

Utgoff, P. E. and Stracuzzi, D. J. (2002). Many-layered learning. Neural Computation, 14(10), 2497-2529.

Vinokourov, A., Shawe-Taylor, J., and Cristianini, N. (2002). Inferring a semantic representation of text via cross-language correlation analysis. In Proceedings of the 15th International Conference on Neural Information Processing Systems, NIPS'02, pages 1497-1504, Cambridge, MA, USA. MIT Press. 
Vossen, P., editor (1998). EuroWordNet: A Multilingual Database with Lexical Semantic Networks. Kluwer Academic Publishers.

Wang, X., Broder, A., Gabrilovich, E., Josifovski, V., and Pang, B. (2008). Crosslingual query classification: A preliminary study. In Proceedings of iNEWS, pages $101-104$.

Xu, J. and Croft, W. B. (1996). Query expansion using local and global document analysis. In Proceedings of the 19th annual international ACM SIGIR conference on Research and development in information retrieval, SIGIR '96, pages 4-11, New York, NY, USA. ACM.

Yang, Y. and Pedersen, J. O. (1997). A comparative study on feature selection in text categorization. In Proceedings of the Fourteenth International Conference on Machine Learning, ICML '97, pages 412-420, San Francisco, CA, USA. Morgan Kaufmann Publishers Inc.

Yih, W., Toutanova, K., Platt, J. C., and Meek, C. (2011). Learning discriminative projections for text similarity measures. In Proceedings of the Fifteenth Conference on Computational Natural Language Learning, CoNLL 2011, Portland, Oregon, USA, June 23-24, 2011, pages 247-256.

Zhou, D., Truran, M., Brailsford, T., Wade, V., and Ashman, H. (2012). Translation techniques in cross-language information retrieval. ACM Computing Surveys, 45(1), 1:1-1:44.

Zobel, J. and Dart, P. (1996). Phonetic string matching: Lessons from information retrieval. In Proceedings of the 19th Annual International ACM SIGIR Conference on Research and Development in Information Retrieval, SIGIR '96, pages 166-172, New York, NY, USA. ACM.

Zobel, J. and Moffat, A. (2006). Inverted files for text search engines. ACM Computing Surveys, $\mathbf{3 8}(2)$. 
Zou, W. Y., Socher, R., Cer, D. M., and Manning, C. D. (2013). Bilingual word embeddings for phrase-based machine translation. In Proceedings of the 2013 Conference on Empirical Methods in Natural Language Processing, EMNLP 2013, 18-21 October 2013, Grand Hyatt Seattle, Seattle, Washington, USA, A meeting of SIGDAT, a Special Interest Group of the ACL, pages 1393-1398. 


\section{Appendix}

\section{A. Gradient derivation}

In this appendix, we derive the gradient calculations for the objective functions in Eq. 4.10 and Eq. 4.12. We first show the gradient derivation for the monolingual pre-initialisation, and then, it is extended for the cross-language extension model.

\section{A.1 Monolingual pre-initialisation}

The parameters of the monolingual pre-initialisation model are shared among the data points: $x_{Q}, x_{D^{+}}$and $x_{D^{-}}$as shown in Fig. 4.6. As each of them contribute to the objective function in Eq. 4.10, the gradient can be derived as follows:

$$
\frac{\partial J(\theta)}{\partial \theta}=\frac{\partial J(\theta)}{\partial \theta_{Q}}+\frac{\partial J(\theta)}{\partial \theta_{D^{+}}}+\frac{\partial J(\theta)}{\partial \theta_{D^{-}}}
$$

where

$$
\frac{\partial J(\theta)}{\partial \theta_{Q}}=\frac{\partial \cos \left(y_{Q}, y_{D^{+}}\right)}{\partial \theta_{Q}}-\frac{\partial \cos \left(y_{Q}, y_{D^{-}}\right)}{\partial \theta_{Q}}
$$

In the deep neural network architecture, the $\theta$ is composed of multiple layer parameters (weights and biases). For example, the gradient of the cosine similarity 
terms in Eq. 2 at the output layer $\left(L_{m}\right)$ w.r.t. the weight matrix $W_{m}$ with $\tanh$ activation can be obtained as follows considering query $Q$ and a document $D$ :

$$
\begin{aligned}
\frac{\partial \cos \left(y_{Q}, y_{D}\right)}{\partial \theta_{Q}^{W_{m}}} & =\frac{\partial}{\partial \theta_{Q}^{W_{m}}} \frac{y_{Q}^{T} y_{D}}{\left\|y_{Q}\right\|\left\|y_{D}\right\|} \\
& =\left[\left(1-y_{Q}\right) \cdot *\left(1+y_{Q}\right) \cdot * \delta_{Q}^{W_{m}}\right] y_{Q}^{L_{m-1}}
\end{aligned}
$$

where.$*$ represents element-wise multiplication, and

$$
\begin{aligned}
\delta_{Q}^{W_{m}} & =\frac{1}{\left\|y_{D}\right\|} \frac{\partial}{\partial \theta_{Q}} \frac{y_{Q}^{T} y_{D}}{\left\|y_{Q}\right\|} \\
& =\frac{1}{\left\|y_{D}\right\|}\left(\frac{\left\|y_{Q}\right\| y_{D}-\left(y_{Q}^{T} y_{D}\right) \frac{y_{Q}}{\left\|y_{Q}\right\|}}{\left\|y_{Q}\right\|^{2}}\right) \\
& =\frac{1}{\left\|y_{D}\right\|} \frac{1}{\left\|y_{Q}\right\|} y_{D}-y_{Q}^{T} y_{D} \frac{1}{\left\|y_{D}\right\|} \frac{1}{\left\|y_{Q}\right\|^{3}} y_{Q}
\end{aligned}
$$

For clear representation, let scalars $y_{Q}^{T} y_{D}, \frac{1}{\left\|y_{Q}\right\|}$ and $\frac{1}{\left\|y_{D}\right\|}$ as $a, b$ and $c$ respectively. Then,

$$
\frac{\partial \cos \left(y_{Q}, y_{D}\right)}{\partial \theta_{Q}^{W_{m}}}=\left[\left(1-y_{Q}\right) \cdot *\left(1+y_{Q}\right) \cdot *\left(b c y_{D}-a c b^{3} y_{Q}\right)\right] y_{Q}^{L_{m-1}}
$$

Similarly, the gradient computation w.r.t. document $D$ is:

$$
\frac{\partial \cos \left(y_{Q}, y_{D}\right)}{\partial \theta_{D}^{W_{m}}}=\left[\left(1-y_{D}\right) \cdot *\left(1+y_{D}\right) \cdot *\left(b c y_{Q}-a c^{3} b y_{D}\right)\right] y_{D}^{L_{m-1}}
$$

Putting all together, Eq. 2 becomes:

$$
\frac{\partial J(\theta)}{\partial \theta_{Q}^{W_{m}}}=\left[\left(1-y_{Q}\right) \cdot *\left(1+y_{Q}\right) \cdot *\left(b c_{p} y_{D^{+}}-a_{p} c_{p} b^{3} y_{Q}-b c_{n} y_{D^{-}}+a_{n} c_{n} b^{3} y_{Q}\right)\right] y_{Q}^{L_{m-1}}
$$


where $a_{p}=y_{Q}^{T} y_{D^{+}}, c_{p}=\frac{1}{\left\|y_{D^{+}}\right\|}, a_{n}=y_{Q}^{T} y_{D^{-}}, c_{n}=\frac{1}{\left\|y_{D^{-}}\right\|}$.

and, w.r.t. $D$ :

$$
\begin{aligned}
& \frac{\partial J(\theta)}{\partial \theta_{D^{+}}^{W_{m}}}=\left[\left(1-y_{D^{+}}\right) \cdot *\left(1+y_{D^{+}}\right) \cdot *\left(b c_{p} y_{Q}-a_{p} c_{p} b^{3} y_{D^{+}}\right)\right] y_{D^{+}}^{L_{m-1}} \\
& \frac{\partial J(\theta)}{\partial \theta_{D^{-}}^{W_{m}}}=-\left[\left(1-y_{D^{-}}\right) \cdot *\left(1+y_{D^{-}}\right) \cdot *\left(b c_{n} y_{Q}-a_{n} c_{n} b^{3} y_{D^{-}}\right)\right] y_{D^{-}}^{L_{m-1}}
\end{aligned}
$$

Similarly for hidden layers, the gradients can be obtained through backpropagation.

\section{A.2 Cross-lingual extension}

The parameters of $\mathrm{CM}_{l_{2}}$ are fixed during the cross-lingual extension training, only the parameters of $\mathrm{CM}_{l_{1}}$ contribute to the objective function in Eq. 4.12. Hence, the derivative of the objective function is obtained as follows:

$$
\begin{aligned}
\frac{\partial J_{c l}(\theta)}{\partial \theta} & =\frac{\partial J_{c l}(\theta)}{\partial \theta_{l_{1}}} \\
& =\frac{\partial \cos \left(y_{l_{1}}, y_{l_{2}}^{+}\right)}{\partial \theta_{l_{1}}}-\frac{\partial \cos \left(y_{l_{1}}, y_{l_{2}}^{-}\right)}{\partial \theta_{l_{1}}}
\end{aligned}
$$

According to Eq. 7, the gradient at the output layer $\left(L_{m}\right)$ of $\mathrm{CM}_{l_{1}}$ w.r.t. $W_{m}$ can be obtained as follows:

$$
\frac{\partial J_{c l}(\theta)}{\partial \theta_{l_{1}}^{W_{m}}}=\left[\left(1-y_{l_{1}}\right) \cdot *\left(1+y_{l_{1}}\right) \cdot *\left(b c_{p} y_{l_{2}}^{+}-a_{p} c_{p} b^{3} y_{l_{1}}-b c_{n} y_{l_{2}}^{-}+a_{n} c_{n} b^{3} y_{l_{1}}\right)\right] y_{l_{1}}^{L_{m-1}}
$$

where $a_{p}=y_{l_{1}}^{T} y_{l_{2}}^{+}, c_{p}=\frac{1}{\left\|y_{l_{2}}^{+}\right\|}, a_{n}=y_{l_{1}}^{T} y_{l_{2}}^{-}, c_{n}=\frac{1}{\left\|y_{l_{2}}^{-}\right\|}$. 


\section{B. Publications}

The research presented in this dissertation has been published in many peer-reviewed conferences and journals. The models, $\mathrm{CAE}$ and XCNN, presented in Chapter 4 are published at (Gupta et al. 2014) and (Gupta et al., 2016a), respectively. The experiments for CLIR applications presented in Chapter 6 are published at various places: ad-hoc and parallel sentence retrieval tasks at (Gupta et al., 2016a), cross-language plagiarism detection at (Barrón-Cedeño et al., 2013; Franco-Salvador et al., 2013, 2016) and source context feature for machine translation at (Gupta et al., 2016b). The research on mixed-script IR presented in Chapter 5 is published at Gupta et al. (2014); Gupta (2014). The work on critical bottleneck dimensionality and related metrics presented in Chapter 7 is published at (Gupta et al., 2016c).

The publications related to the work on this dissertation are listed below:

1. Gupta, P., Bali, K., Banchs, R. E., Choudhury, M., and Rosso, P. (2014). Query expansion for mixed-script information retrieval. In Proceedings of the 37th International ACM SIGIR Conference on Research \& Development in Information Retrieval, SIGIR '14, pages 677-686, New York, NY, USA. ACM

2. Gupta, P. (2014). Modelling of terms across scripts through autoencoders. In Proceedings of the 37th International ACM SIGIR Conference on Research 8 Development in Information Retrieval, SIGIR '14, pages 1279-1279, New York, NY, USA. ACM

3. Gupta, P., Banchs, R. E., and Rosso, P. (2016a). Continuous space models for CLIR. Information Processing \& Management, 53(2), 359-370

4. Gupta, P., Costa-Jussà, M. R., Rosso, P., and Banchs, R. E. (2016b). A deep source-context feature for lexical selection in statistical machine translation. Pattern Recognition Letters, 75, 24-29

5. Gupta, P., Banchs, R. E., and Rosso, P. (2016c). Squeezing bottlenecks: Ex- 
ploring the limits of autoencoder semantic representation capabilities. Neurocomputing, 175, 1001-1008

6. Barrón-Cedeño, A., Gupta, P., and Rosso, P. (2013). Methods for crosslanguage plagiarism detection. Knowledge-Based Systems, 50, 211-217

7. Franco-Salvador, M., Gupta, P., and Rosso, P. (2013). Cross-language plagiarism detection using a multilingual semantic network. In Proceedings of the 35th European Conference on Advances in Information Retrieval, ECIR'13, pages 710-713, Berlin, Heidelberg. Springer-Verlag

8. Franco-Salvador, M., Gupta, P., Rosso, P., and Banchs, R. E. (2016). Crosslanguage plagiarism detection over continuous-space- and knowledge graphbased representations of language. Knowledge-Based Systems, 111, 87-98

9. Gupta, P., Rosso, P., and Banchs, R. E. (2013a). Encoding transliteration variation through dimensionality reduction: FIRE Shared Task on Transliterated Search. In Fifth Forum for Information Retrieval Evaluation, FIRE '13

10. Costa-jussà, M. R., Gupta, P., Rosso, P., and Banchs, R. E. (2014). Englishto-hindi system description for WMT 2014: Deep source-context features for Moses. In Proceedings of the Ninth Workshop on Statistical Machine Translation, WMT '14, pages 79-83, Baltimore, Maryland, USA. Association for Computational Linguistics

The publications that are partially related to the dissertation and obtained during the course of the $\mathrm{PhD}$ are listed below:

1. Sequiera, R., Choudhury, M., Gupta, P., Rosso, P., Kumar, S., Banerjee, S., Naskar, S. K., Bandyopadhyay, S., Chittaranjan, G., Das, A., and Chakma, K. (2015). Overview of FIRE-2015 shared task on mixed script information retrieval. In Seventh Forum for Information Retrieval Evaluation, FIRE '15, pages $19-25$ 
2. Dubey, A., Gupta, P., Varma, V., and Rosso, P. (2014). Enrichment of bilingual dictionary through news stream data. In Proceedings of the Ninth International Conference on Language Resources and Evaluation, LREC '14, pages 37613765

3. Choudhury, M., Chittaranjan, G., Gupta, P., and Das, A. (2014). Overview of FIRE 2014 track on transliterated search. In Sixth Forum for Information Retrieval Evaluation, FIRE '14

4. Gupta, P., Clough, P. D., Rosso, P., Stevenson, M., and Banchs, R. E. (2013b). PAN@FIRE: Overview of the Cross-Language !ndian News Story Search (CL!NSS) track. In Fifth Forum for Information Retrieval Evaluation, FIRE '13

5. Gupta, P. and Rosso, P. (2012a). Expected divergence based feature selection for learning to rank. In Proceedings of the 24th International Conference on Computational Linguistics, COLING '12, pages 431-440

6. Gupta, P. and Rosso, P. (2012b). Text reuse with ACL: (Upward) Trends. In Proceedings of the ACL-2012 Special Workshop on Rediscovering 50 Years of Discoveries, ACL '12, pages 76-82, Stroudsburg, PA, USA. Association for Computational Linguistics

7. Gupta, P., Barrón-Cedeño, A., and Rosso, P. (2012b). Cross-language high similarity search using a conceptual thesaurus. In Proceedings of the Third International Conference on Information Access Evaluation: Multilinguality, Multimodality, and Visual Analytics, CLEF '12, pages 67-75, Berlin, Heidelberg. Springer-Verlag

8. Potthast, M., Gollub, T., Hagen, M., Kiesel, J., Michel, M., Oberländer, A., Tippmann, M., Barrón-Cedeño, A., Gupta, P., Rosso, P., and Stein, B. (2012). Overview of the 4th international competition on plagiarism detection. In CLEF Evaluation Labs and Workshop, Online Working Notes, CLEF '12 